\title{
Burundi: 2012 Article IV Consultation and First Review Under the Three-Year Arrangement Under the Extended Credit Facility and Request for Modification of Performance Criteria-Staff Report; Public Information Notice and Press Release on the Executive Board Discussion; and Statement by the Executive Director for Burundi.
}

Under Article IV of the IMF's Articles of Agreement, the IMF holds bilateral discussions with members, usually every year. In the context of a combined discussion of the 2012 Article IV consultation with Burundi and first review under the three-year arrangement under the Extended Credit Facility and request for modification of performance criteria, the following documents have been released and are included in this package:

- The staff report for the combined 2012 Article IV Consultation and first review under the threeyear arrangement under the Extended Credit Facility and request for modification of performance criteria, prepared by a staff team of the IMF, following discussions that ended on June 8, 2012, with the officials of Burundi on economic developments and policies. Based on information available at the time of these discussions, the staff report was completed on July 12, 2012. The views expressed in the staff report are those of the staff team and do not necessarily reflect the views of the Executive Board of the IMF.

- $\quad$ A Public Information Notice (PIN) and Press Release, summarizing the views of the Executive Board as expressed during its July 27, 2012, discussion of the staff report on issues related to the Article IV consultation and the IMF arrangement, respectively.

- $\quad$ A statement by the Alternate Executive Director for Burundi.

The documents listed below have been or will be separately released.

Joint Staff Advisory Note of the Poverty Reduction Strategy Paper (PRSP II)

Letter of Intent sent to the IMF by the authorities of Burundi*

Memorandum of Economic and Financial Policies by the authorities of Burundi*

Poverty Reduction Strategy Paper (PRSP II)

Technical Memorandum of Understanding*

*Also included in Staff Report

The policy of publication of staff reports and other documents allows for the deletion of market-sensitive information.

Copies of this report are available to the public from International Monetary Fund • Publication Services $70019^{\text {th }}$ Street, N.W. $\bullet$ Washington, D.C. 20431

Telephone: (202) 623-7430 • Telefax: (202) 623-7201

E-mail: publications@imf.org Internet: http://www.imf.org

\section{International Monetary Fund Washington, D.C.}




\section{INTERNATIONAL MONETARY FUND}

\section{BURUNDI}

\section{STAFF REPORT FOR THE 2012 ARTICLE IV CONSULTATION, FIRST REVIEW UNDER THE THREE YEAR ARRANGEMENT UNDER THE EXTENDED CREDIT FACILITY AND REQUEST FOR MODIFICATION OF PERFORMANCE CRITERIA}

July 12, 2012

\section{KEY ISSUES}

Context: Despite a difficult economic and social context, the government has made steady, though uneven, progress in implementing its ECF-supported economic reforms. Civil society and trade unions have been restive given high inflation due to the persistence of food and fuel shocks.

Program: All end-March 2012 quantitative targets were met. However, fiscal slippages emerged requiring corrective fiscal measures (about $1 \frac{112}{2}$ percent of GDP). The implementation of structural reforms was uneven. Continued efforts in revenue mobilization are critical in addressing declining donor support, while ongoing strengthening of public financial and debt management augur well for transparency and improved governance.

Outlook and risk: The medium-term macroeconomic outlook is challenging. Risks emanate from a delicate social situation given persistent shocks and the high cost of living. Declining donor support, if sustained, would imply greater fiscal adjustment in realigning spending with available resources while external spillovers through the trade channel could weaken demand for exports.

Staff views: The staff recommends completion of the review and disbursement of SDR 4 million, and modification of performance criteria and indicative targets for endSeptember 2012. 
Approved By

Roger Nord and

Dhaneshwar Ghura
A team comprising Messrs. Williams (head), Boutin-Dufresne, Peña (all AFR), and Roitman (FAD) visited Bujumbura during May 28-June 8, 2012. Mr. Yao (Resident Representative) assisted the mission and participated in the discussions. Mr. Majoro (ED) participated at the end of the mission. The mission met with Second Vice President Rufyikiri, President of the Senate Ntsizerana, Minister of Finance Manirakiza, Central Bank Governor Sindayigaya, other senior government officials, and representatives of labor unions, the private sector, CSOs, the donor community, and the media.

\section{CONTENTS}

BACKGROUND, RECENT DEVELOPMENTS AND PROGRAM IMPLEMENTATION 4

A. Background__ 4

B. Recent Developments ___ 4

C. Program Implementation__ 5

MACROECONOMIC OUTLOOK AND RISKS

POLICY THEME \# 1-FORGING AHEAD WITH REVENUE MOBILIZATION 8

POLICY THEME \# 2-BOLSTERING PUBLIC FINANCIAL MANAGEMENT 10

POLICY THEME \# 3-STRENGTHENING DEBT MANAGEMENT

POLICY THEME \# 4-REINING IN INFLATION

POLICY THEME \# 5-REINFORCING FINANCIAL SECTOR SURVEILLANCE

POLICY THEME \# 6-MONITORING INWARD SPILLOVERS 15

POLICY THEME \# 7-INCREASING COMPETITIVENESS 


\section{TABLES}

1. Selected Economic and Financial Indicators, 2010-15 25

2a. Central Government Operations, 2010-15 26

2b. Central Government Operations, 2010-15 27

3. Monetary Survey, 2010-13 _ 28

4. Central Bank Accounts, 2010-12 _ 29

5. Balance of Payments, 2010-15___ 30

6. Indicators of Capacity to Repay the Fund, 2012-20__ 31

7. Actual and Projected Schedule of ECF Disbursements and Reviews, 2012-15___ 32

8. Banking System Soundness Indicators, 2009-12 __ 33

\section{FIGURES}

1. Inflation Developments Corrected for One-off Effects___ 4

2. Tax Revenue before and after Reforms __ 10

3. Growth Correlation _ 15

4. Net Risk Exposure of BIS Banks to Burundi ___ 16

5. External Loans and Deposits of Reporting Banks vis-à-vis the Banking Sector in Burundi___ 16

6. Real and Equilibrium Exchange Rate, 2000-11___ 17

7. Relative productivity and REER, 2000-11 __ 18

8. Macroeconomic Developments, 2007-2012__ 22

9. Monetary Developments, 2008-2012__ 23

10. EAC: Financial Stability Indicators, 2010-2011__ 24

\section{APPENDIXES}

I. Letter of Intent

Attachment I. Memorandum on Economic and Financial Policies, 2011-14___ 54

Table I-1. Performance Criteria and Indicative Targets for $2012 \ldots 64$

Table I-2. Structural Benchmarks for 2012

Attachment II. Technical Memorandum of Understanding __ 66

II. Draft Public Information Notice___ 73

\section{ANNEXES}

I. Risk Assessment Matrix __ 34

II. Burundi-Should the Fuel Pricing Mechanism be Revised? ___ 36

III. Exchange Rate Assessment ___ 44 


\section{BACKGROUND, RECENT DEVELOPMENTS AND PROGRAM IMPLEMENTATION}

\section{A. Background}

\section{Burundi is one of the poorest}

countries in the world. GDP per capita is about US $\$ 255$, and more than two-thirds of the population lives below the poverty line. While the country is making some progress toward the Millennium Development Goals (MDGs), chances of achieving them by 2015 are still limited.

\section{In a difficult post conflict}

environment, Burundi has made steady, though uneven, progress in implementing its ECF-supported economic reforms. At the

\section{B. Recent Developments}

3. Real GDP growth is estimated to have increased to 4.2 percent in 2011. After decelerating to 15 percent ( $y-0-y)$ toward end2011, headline inflation rose sharply in March 2012 to 24.5 percent ( $y$-o-y) owing to a rise in rents, utility tariffs, and higher food prices. ${ }^{1}$ It has since eased slightly to 22.7 percent in May 2012. However, when corrected for these one off measures, underlying inflation is trending downwards (Figure 1).

\footnotetext{
${ }^{1}$ Water and electricity tariffs were raised in January, the second time in six months, as part of the strategy to strengthen the financial position of the public utility company. The CPI basket covers the capital only.
}

conclusion of the 2010 Article IV consultation, Executive Directors commended the authorities for the satisfactory implementation of the prior ECF-supported program while urging decisive progress on structural reforms to accelerate growth and reduce poverty.

\section{Notwithstanding recent progress in}

nation building, the social situation remains fragile. Civil society and trade unions conducted a strike to protest increases in electricity tariffs and fuel prices and high inflation due to the persistence of food and fuel shocks.

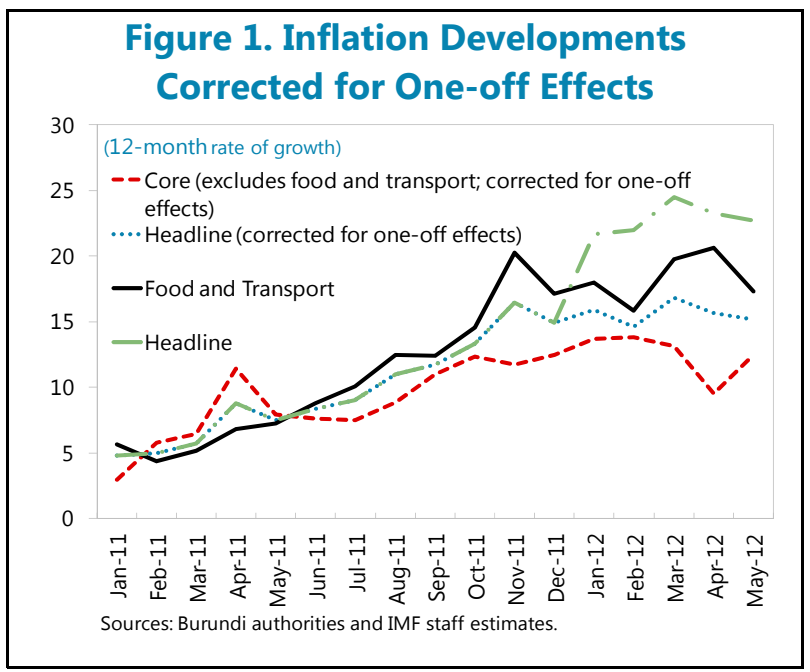


5. Fiscal trends. While revenues and expenditure were broadly in line with the program, the deficit in 2011 was higher than expected due to the late disbursement of the World Bank budget support operation. ${ }^{2}$ Revenues through end-May 2012 were lower than expected by ( 0.5 percent of GDP), owing to a fall in collections of excise taxes and the granting of exemptions. To limit the impact of inflation on the poor, the government eliminated taxes on food products on May 1 until end-2012.

\section{Money and credit. Growth in broad} money decelerated to about 4.7 percent (y-o-y) in April 2012 following tighter monetary conditions as policy rates rose by 350 basis points to 14 percent in May 2012. Credit to the private sector after peaking at 40 percent at end-2011 slowed to 28 percent (y-o-y) at end-April 2012 in the face of tighter liquidity conditions. Banks were able to sustain credit growth despite a decline in deposits, in part through a drawdown in excess reserves.

\footnotetext{
${ }^{2}$ The budget support operation expected in endDecember 2011 was disbursed in January 2012 due to delays.
}

\section{Program Implementation}

\section{Program performance has been challenging under difficult economic conditions (MEFP, Tables I.1-I.2).}

All quantitative and indicative targets for end-December 2011 except that on pro-poor spending and all performance criteria and indicative targets for end-March 2012 were observed. Structural benchmarks for the first review are still pending. The decree on budget governance that realigns current regulation with the new organic law is awaiting presidential approval, while recruitment for the international firm to conduct an audit of the central bank was delayed due to the need to incorporate the recommendations of the safeguards assessment mission into the terms of reference. The safeguards recommendations were incorporated into the terms of reference and bids for the selection of the auditors have now been received and selection is envisaged by mid-July. Submission of the auditor's report is now expected by end-September in light of the delays (LOI $\llbracket 4$, MEFP $\llbracket 21$ ).

Regarding the decree on budget governance clarifications were incorporated into the legal text, reflecting discussions by the Council of Ministers. These changes will be reviewed shortly by the Council of Ministers prior to signature by the President in mid-July (LOI $\llbracket 4$ and MEFP ॠ25). 
Text Table 1. Structural Benchmarks for First Review

\begin{tabular}{lll}
\hline Measure & Dates & Status \\
\hline $\begin{array}{l}\text { Adopt decree } \\
\text { on budget } \\
\text { governance }\end{array}$ & June 30,2012 & $\begin{array}{l}\text { Awaiting Presidential } \\
\text { signature }\end{array}$ \\
& & $\begin{array}{l}\text { Delayed pending } \\
\text { incorporation of }\end{array}$ \\
& & $\begin{array}{l}\text { safeguards } \\
\text { recommendations in } \\
\text { the TOR }\end{array}$ \\
$\begin{array}{l}\text { Recruit an } \\
\text { international } \\
\text { auditor }\end{array}$ & March 31, 2012 & $\begin{array}{l}\text { Delayed pending the } \\
\text { recruitment of the } \\
\text { international auditor }\end{array}$ \\
$\begin{array}{l}\text { Submit report } \\
\text { on special audit }\end{array}$ & June 30, 2012 & \\
\hline
\end{tabular}

\section{MACROECONOMIC OUTLOOK AND RISKS}

\section{The medium-term macroeconomic}

outlook is challenging. Real GDP growth,

driven by the agricultural, construction and

tourism sectors, and improved electricity

supply, is projected to rise gradually over the

medium term. ${ }^{3}$ The medium-term growth

scenario assumes a successful reform of the

coffee sector and increased value-added in the construction and tourism sectors. Headline

inflation is expected to decline to around

15 percent by end-2012, owing to continued

monetary tightening and an expected

improvement in harvests and narrow over the

medium term. The fiscal balance will initially

worsen as construction of large hydroelectric

plants is implemented and budget support

declines (Table 2b). The current account

\footnotetext{
${ }^{3}$ The tourism sector is emerging as a growth center with key linkages to agriculture.
}

balance is projected to slowly narrow over the medium term, driven by lower oil prices. Higher coffee exports are also projected to contribute to this improvement. ${ }^{4}$ The macro framework is predicated on identified financing and is less optimistic than that proposed in the PRSP.

Text Table 2. Burundi: Medium-Term Macroframework

\begin{tabular}{lrrrrr}
\hline & 2011 & 2012 & 2013 & 2014 & 2015 \\
\cline { 4 - 7 } & & Prel. & \multicolumn{4}{c}{ Proj. } \\
\hline & \multicolumn{6}{c}{ (Annual percentange change) } \\
& 4.2 & 4.2 & 4.5 & 5.1 & 5.5 \\
Real GDP & 14.9 & 14.7 & 8.4 & 6.1 & 6.4
\end{tabular}

(In percent of GDP)

Fiscal balance $\quad-4.0 \quad-2.7 \quad-4.6 \quad-4.9 \quad-3.9$

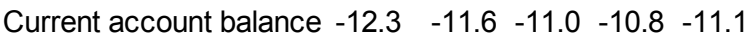
Source: IMF staff estimates and projections.

\footnotetext{
${ }^{4}$ Coffee production conforms to an irregular two-year cycle alternating between high and low harvests.
} 


\section{Risks to the outlook remain}

elevated. A deterioration of the political and security situation, a further decline in donor support, or a worsening in the terms of trade could cause a slowdown in growth, reform implementation, and social unrest. While multilateral donors continue to provide budget support, albeit with stronger conditionality, bilateral donors have become less engaged in part because of fiscal difficulties in home countries (see Risk Assessment Matrix in Annex I) ${ }^{5}$

\footnotetext{
${ }^{5}$ The EU has now linked budget support to political governance, encompassing progress in the areas of rule of law and human rights.
} 


\section{POLICY THEME \# 1-FORGING AHEAD WITH REVENUE MOBILIZATION}

Policy discussions focused on consolidating the gains from recent successful revenue reforms. This primarily requires widening the tax base and recouping revenue losses associated with mitigating the impact of recent shocks on the poor.

\section{Food and fuel shocks have} continued to put pressure on government's operations (Annex II). Persistently high oil prices combined with the partial pass-through to domestic prices are projected to yield losses in excise taxes ( 0.6 percent of GDP). The revenue loss associated with the elimination of taxes on food products are estimated at BIF 10 billion ( 0.3 percent of GDP). Disruptions to domestic food supply due to inclement weather exacerbated the persistence of food inflation. In response, the authorities announced the elimination of taxes on some food products until the end of the year.

Slippages on the expenditure front estimated at about 0.5 percent of GDP were mostly related to weak control of the wage bill and higher outlays in the health sector.

\section{The authorities undertook corrective} measures to address the revenue shortfalls and expenditure slippages. These included revenue measures comprising an increase in taxes on income, excise taxes on alcohol, tobacco, telecoms and duties on used cars
(0.8 percent of GDP). ${ }^{6}$ The revenue measures will not fully compensate for revenue slippages, requiring additional expenditure measures. Further revenue measures in a difficult economic and social context could prove to be challenging ( $\pi 4$ and $\pi 48$ ). The authorities also undertook expenditure cuts amounting to 0.5 percent of GDP, with domestically financed capital spending accounting for the lionshare. The authorities also benefitted from higher privatization receipts (MEFP \13) from the sale of coffee washing stations and other assets ( 0.2 percent of GDP).

Text Table 3. Fiscal slippages and Corrective Measures, 2012

\begin{tabular}{lcc}
\hline & BIF billions & In percent of GDP \\
\hline Fiscal slippage & $\mathbf{5 0 . 8}$ & $\mathbf{1 . 4}$ \\
Losses in excise taxes & 22.9 & 0.6 \\
Losses in taxes on food & 10.0 & 0.3 \\
Expenditure overruns & 17.9 & 0.5 \\
Total measures & $\mathbf{5 0 . 8}$ & $\mathbf{1 . 4}$ \\
Revenue & 26.9 & 0.8 \\
Expenditure cuts & 17.9 & 0.5 \\
Privatization proceeds & 6 & 0.2 \\
\hline Source: Burundi Authorities and IMF Staff estimates.
\end{tabular}

\footnotetext{
${ }^{6}$ The increase in excise taxes brings them in closer alignment with those of East African Community as part of the authorities' commitments.
} 


\section{The continued implementation of} revenue and tax reforms aims to consolidate the gains from past efforts, to compensate for declining budget support, and to rebuild buffers. Budget support has been on a declining trend from 5 percent of GDP in 2010 to about 3 percent of GDP in 2012, necessitating continued momentum in revenue mobilization. Burundi's revenue reforms compares favorably with those undertaken by other developing countries, including El Salvador, Tanzania, and Vietnam. ${ }^{7}$ Reforms are two-pronged: comprising (i) widening the tax base through the introduction of an excise tax law and eliminating discretionary exemptions; and (ii) strengthening revenue administration. $\mathrm{A}$ review of legal texts granting tax exemptions ahead of introducing the income tax law will ensure consistency and identify the level of tax expenditures.

\section{Staff underscored the need to} sustain the momentum in implementing revenue reforms. Although the Burundi Revenue Authority (BRA) has been successful in widening the tax base and improving revenue collections, slippages in the pace of collections have emerged. Sustaining revenue reforms is crucial in light of declining budget

\footnotetext{
${ }^{7}$ See IMF (2011) "Revenue Mobilization in Developing Countries (SM/11/21)
}

support and the need to recoup recent revenue losses. There is further scope to improve collections on excise taxes and taxes on incomes and profits. Staff stressed that the existing fuel pricing mechanism is inefficient and presented a number of alternatives.

\section{Authorities' Views}

\section{The authorities reiterated their commitment to pursue revenue reforms.}

They acknowledged that the Burundi Revenue Authority (BRA) was a successful reform that demonstrated the results that could be achieved in concert with development partners. They noted that recent exemptions on food products were temporary and driven by the need to alleviate the impact of the shocks on the poor. They also underscored their determination to limit exemptions to those inherent to the investment code and that exemptions policy will be addressed in the context of the reforms (September benchmark). The authorities recognized that the existing fuel pricing mechanism was costly and requested technical assistance (TA) to put in place a fuel pricing mechanism with fixed price bands. $^{8}$

\footnotetext{
${ }^{8}$ Fixed price bands permit less price volatility at the pump and mitigate revenue losses.
} 


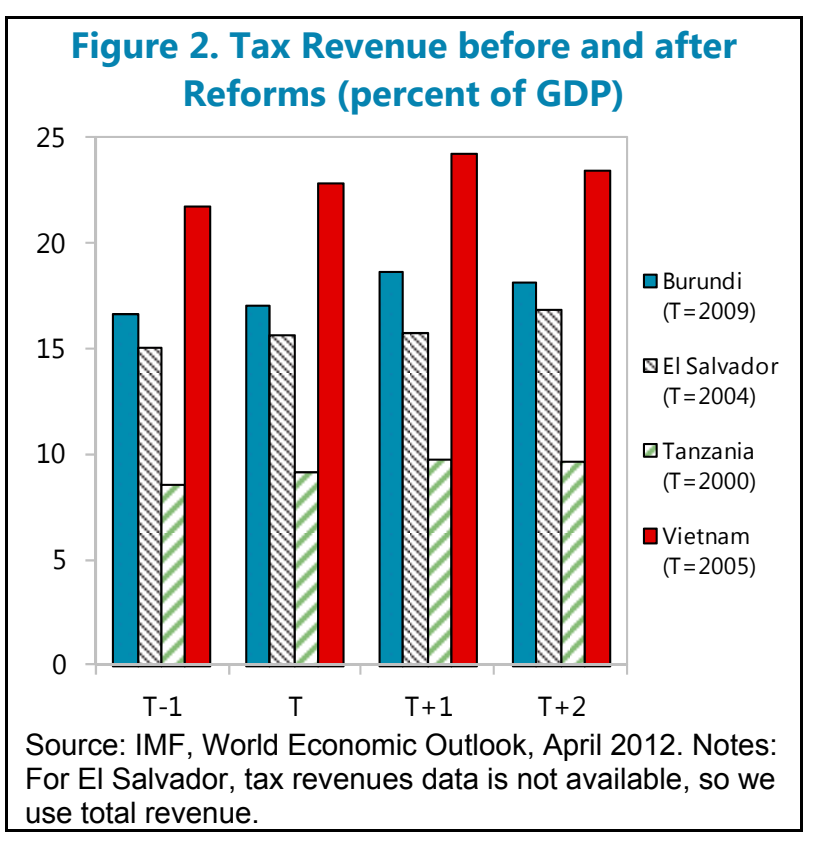

\section{POLICY THEME \# 2-BOLSTERING PUBLIC FINANCIAL MANAGEMENT}

Sound public financial management (PFM) remains a cornerstone of the program that aims to improve financial accountability and transparency. Strengthening institutional capacity through technical assistance (TA) will enhance budget design, execution, and monitoring.

\section{Improving governance and} transparency are important aspects of donor conditionality. A recent evaluation under the Public Expenditure and Financial Accountability (PEFA) framework found that progress was made in reinforcing the credibility of the budget. A new PFM strategy, based on the results of the PEFA evaluation and IMF technical assistance, was adopted by the Council of Ministers. The authorities will adopt the decree on governance to align the current regulation and procedures with the
2008 Organic Budget Law. This decree will facilitate the deployment of commitment controllers in two pilot ministries (Agriculture and Health). They reiterated that the decree clarifying the roles of the BRA and the Accountant General will be adopted shortly (MEFP ॠ24).

\section{Rapid implementation of PFM} reforms would foster greater transparency and accountability of public finances and encourage donor support (MEFP \24). The PFM strategy proposes the adoption of a 
multi-year approach to budget preparation for 2012-15, modernization of the legal framework, better control of budget execution, including the wage bill, and strengthening the institutional arrangements governing the BRA. The implementation of the strategy will be supported by TA provided by the IMF and other development partners, with a focus on training and capacity building.

\section{Strengthening the financial} operations of public enterprises is essential to reinforce safeguards and limit budget transfers (MEFP П28). The authorities increased water and electricity tariffs of the state utility company to place its finances on a sound footing. About half of its 60,000 customers have been fitted with pre-paid meters, and an additional 15,000 are envisaged by end-2012. An audit of its operations is being conducted as part of the World Bank's reform of the electricity sector.

\section{Staff stressed the importance of} adhering to the implementation schedule underpinning reforms. The sequencing of reforms is a prerequisite for the full implementation of the legal texts governing the 2008 Organic Budget Law. In addition, staff underscored the role of TA in strengthening capacity through hands-on training in order to ensure the success of the reforms.

\section{Authorities' Views}

\section{The authorities reiterated their} commitment to implement PFM reforms. They emphasized the need for a long-term expert to help implement the reforms. They also noted that better control of recruitment through the centralization of the process is essential to mitigating risks posed by the wage bill.

\section{POLICY THEME \# 3-STRENGTHENING DEBT MANAGEMENT}

Preserving debt sustainability is a key objective of the program and is the anchor of medium-term fiscal policy.

20. Given its narrow export base and limited capacity, Burundi remains at a high risk of debt distress. ${ }^{9}$ The public debt sustainability analysis conducted in January

${ }^{9}$ The last Debt Sustainability Assessment produced
jointly by the IMF and World Bank staffs was presented (continued) 
2012 suggests that while Burundi is in a position to service its public and publicly guaranteed debt, lower economic growth would lead to a considerable deterioration of debt indicators. The latest data do not materially change this outcome.

\section{The recommendations of a recently} conducted Debt Management Performance Assessment (DEMPA) are key inputs for the medium-term strategy (MEFP I25). The authorities undertook a DEMPA evaluation with a view to designing a medium-term strategy (September benchmark). Compared to the 2009 exercise, progress was noted in strengthening the legal framework, implementation of an external audit, adoption of a clearer separation between monetary policy and financing fiscal operations, and the creation of an up-to-date securities register at the central bank. Notwithstanding, important gaps remain in the formulation of a debt management strategy, procedures for guarantees and on-lending, data management, and the regularity of reporting.

\section{A re-organization of the ministry of} finance will be needed to better manage debt related issues. Strengthening the debt management unit at the ministry of finance,

to the Board in January 2012 (IMF Country Report No. 12/28). and its integration with treasury operations should be at the center of the new debt management strategy. This reform is a prerequisite for reinforcing coordination between the ministry of finance and the central bank on determining treasury cash flow and financing needs by better accounting for liquidity and macroeconomic developments. The mission recommended the reactivation of the joint committee comprising the central bank and the ministry of finance responsible for the monitoring and execution of the budget and its financing.

\section{Staff emphasized that Burundi} should continue to implement prudent borrowing policies and accelerate structural reforms for private sector led growth, particularly in infrastructure and export diversification, to mitigate risks to debt sustainability. The fiscal anchor for the program continues to be predicated on a zero limit on non concessional external public borrowing and a gradually declining path for domestic debt to GDP.

\section{Authorities' Views}

\section{The authorities recognized the risks} of debt distress noting that the new PRSP reaffirms the authorities' commitment to continue to rely on grants and highly concessional loans. 


\section{POLICY THEME \# 4-REINING IN INFLATION}

Monetary policy continues to focus on anchoring inflation expectations by containing the entrenchment of second round effects associated with the persistent food and fuel shocks.

\section{The effectiveness of monetary policy} is stymied by a several structural factors.

These include a rudimentary interbank market, the monopoly role of the central bank in providing foreign exchange, and structural excess liquidity related to low statutory reserve requirements (3 percent) which constrain the efficacy of the interest rate channel, Further TA is needed in developing policy instruments for financing the deficit and for open market operations.

\section{Tighter liquidity conditions have} dampened credit growth from its peak in 2011(โ6). Policy rates have risen in line with those in the East African Community whose members are facing a common shock. The rise in policy interest rates has helped to reduce the growth in credit as liquidity conditions have tightened. In addition, the transmission of monetary policy has improved as excess structural liquidity in the banking system declined. However, structural factors constrain the efficacy of liquidity management. There are limits to which the central bank as the major provider of foreign exchange could use foreign exchange sales to mop up excess liquidity in light of declining donor inflows.

\section{Monetary policy should be} tightened further as inflation remains stubbornly high. There is room for further tightening as real interest rates remain negative. Slower growth in credit to the private sector from previous peaks suggest that aggregate demand has begun to cool down in the context of tighter liquidity conditions.

\section{Authorities' Views}

\section{The authorities did not see the need} for further monetary tightening at this point. The authorities emphasized that supply shocks are the main drivers of inflation, and that prospects for improved harvests commencing in June and the easing of taxes on food imports would attenuate inflationary pressures. They underscored that the low growth in monetary aggregates and the declining path of credit to the private sector are conducive to a slowdown in prices but would stand ready to take additional measures should inflationary pressures not ease during the remainder of the year. 


\section{POLICY THEME \# 5-REINFORCING FINANCIAL SECTOR SURVEILLANCE}

Enhanced surveillance of the financial sector would help mitigate risks arising from an unfavorable global environment, rising credit risk, and the adverse effects of recent shocks.

\section{The banking sector remains sound} despite recent rapid credit growth (Table 9 and Figure 10). Although banks have comfortable liquidity and large capital buffers, rising policy rates and the adverse impact of recent shocks on the economy could give rise to vulnerabilities in the banking and corporate sectors. NPLs remain in single digits but have risen in the face of tougher economic conditions.

\section{Good progress continues toward} bolstering banking supervision (MEFP 112).

Through IMF TA, a manual on risk-based supervision has been developed. The Banking Supervision Department has been restructured to perform financial sector stability analysis. Further reforms in train include the migration of the banking system toward International Financial Reporting Standards by 2013.

\section{Strengthening creditor rights is a} key reform aimed at protecting investors and financial sector deepening. Addressing gaps in the creditor rights framework that lead to the recovery of creditors' claims would facilitate development of the financial sector and its deepening. Difficulties in recovering claims through the judicial system are cited as a major constraint and have led to the lengthening of loan maturities in some cases in order to facilitate repayment.

32. Staff urged the authorities to closely monitor risks to the banking sector arising from a tight monetary stance. Credit to the private sector which grew at a fast pace since 2010, has begun to decelerate. Staff underscored that credit booms tend to mask the buildup of excesses in good times and stressed the need to closely monitor the impact of adverse developments and tighter monetary conditions on banks' balance sheets.

\section{Authorities' Views}

\section{The authorities stressed that} banking supervision will be vigilant in monitoring credit risk that could arise from rising interest rates and adverse external developments. The authorities convened discussions with commercial banks regarding the impacts of adverse developments on their balance sheets and to better assess emerging credit risks. Increased on-site inspections are envisaged. 


\section{POLICY THEME \# 6-MONITORING INWARD SPILLOVERS}

As a small landlocked and open economy, Burundi is vulnerable to external shocks.

\section{Burundi remains vulnerable to}

shocks arising primarily through the trade

channel. Its exports can be categorized into three main clusters, the European Union (EU), Asia, and sub-Saharan Africa (SSA). Within SSA the East African Community (EAC) with whom Burundi has embarked on a process of monetary integration accounts for the majority of exports (10 percent). Rwanda, Kenya, and Tanzania have become key gate keepers for Burundi's exports underscoring that common shocks that confront the region would be an important channel for the transmission of spillovers. Also noteworthy is an increasing shift in the share of exports toward Asia (15 percent) at the expense of the $\mathrm{EU}$ (46 percent).

\section{The correlation between Burundi's} real GDP growth and key trading partners has increased since the crisis. Prior to the crisis, Burundi's real GDP growth and that of some major trading partners were negatively correlated. However, since the global financial crisis the co-movement with the EU has increased suggesting that shocks arising in these countries can be transmitted to Burundi through the trade channel.

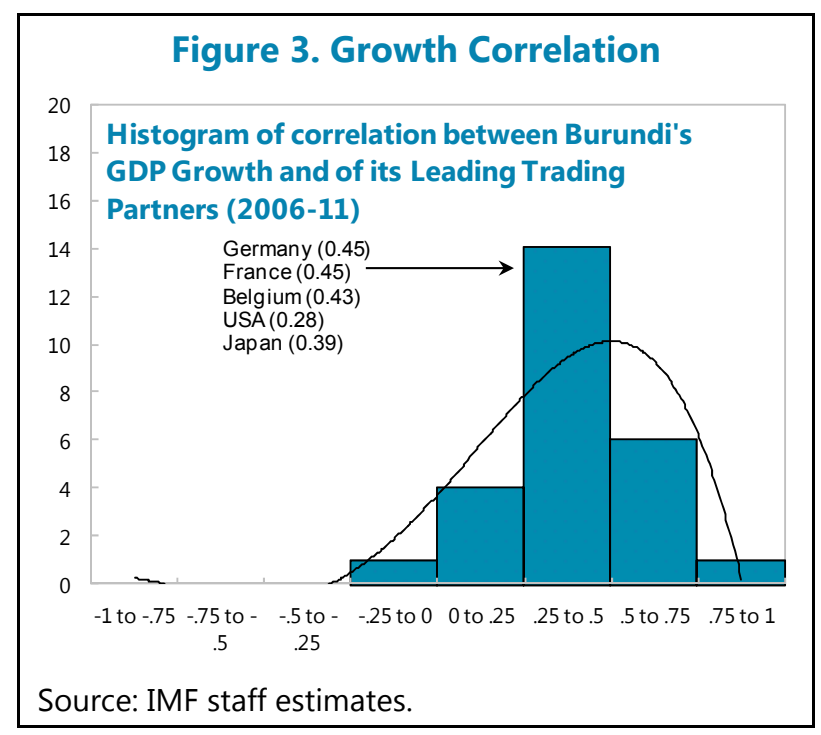

36. According to the most recent data from the Bank of International Settlements (BIS), the risk exposure of BIS banks to Burundi has increased. ${ }^{10}$ The banking system is interconnected with BIS banks through onlending to subsidiaries which subsequently finance operations in Burundi. Although the net exposure of BIS banks has increased during 2011, Burundi is a net creditor to these

\footnotetext{
${ }^{10}$ In line with the risk reallocation principle for measuring country exposure, the country of ultimate risk or where the final risk lies is defined as the country in which the guarantor of a financial claim resides and/or the country in which the head office of a legally dependent branch is located.
} 
banks. Risks to the banking system are mitigated in part because it relies mostly on retail deposits and there is very little wholesale funding. The majority of banks are local with the exception of two Pan-African banks with operations in several African countries.

\section{Figure 4. Net Risk Exposure of BIS Banks} to Burundi

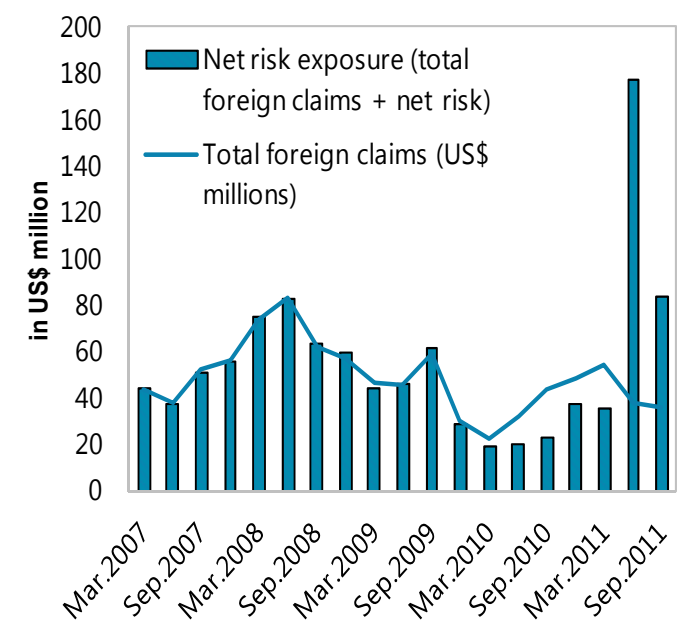

Source: Bank of International Settlements.

Figure 5. External Loans and Deposits of Reporting Banks vis-à-vis the Banking Sector in Burundi (in million of US\$)

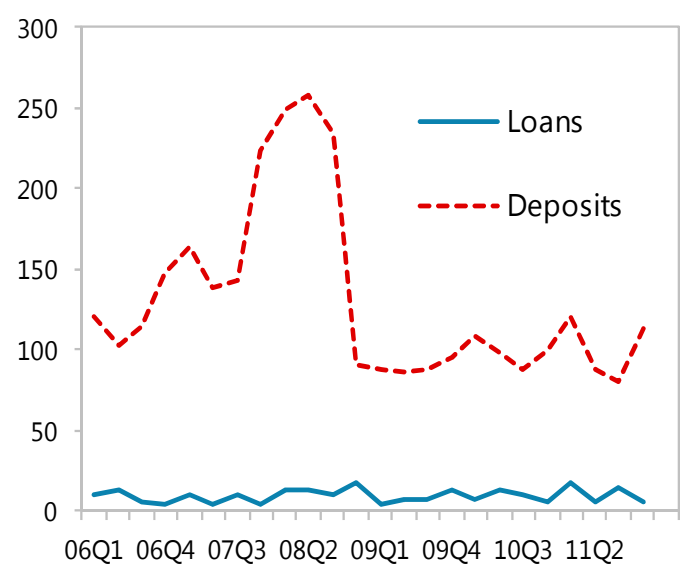

Source: Bank of International Settlements.
37. Staff urged the authorities to remain vigilant in the face of heightened risks emanating from an unfavorable external environment. While the channels for transmitting financial stress are primarily indirect, staff encouraged the authorities to continue monitoring banks whose cross-border liabilities to BIS banks have increased.

\section{Authorities' Views}

\section{The authorities envisaged minor} impact from the Euro zone crisis. They noted that spillovers from the Euro zone crisis were likely transmitted to the trade rather than the financial channel. They underscored that the banking system depended mainly on retail deposits with little recourse to wholesale funding. They pointed out that memoranda of understandings were in place with crossborder supervisors that facilitated supervision of Pan-African banks. 


\section{POLICY THEME \# 7-INCREASING COMPETITIVENESS}

The exchange rate remains an important policy tool in allowing the economy to adjust to external shocks.

\section{Greater flexibility of the exchange} rate facilitated the adjustment to external

shocks. By changing the manner in which the reference exchange rate was calculated to one based on the transactions of commercial banks, the authorities allowed greater movement in the exchange rate by including more market information in the setting of the official exchange rate. As a result, the deviation between the official and market rates narrowed and the currency has been reclassified from "stabilized" to "other managed". These developments facilitated a rebalancing of the policy mix with exchange rate assuming a greater share in the burden of adjustment.

\section{An assessment of the level of the} exchange rate suggests that the Burundi Franc is slightly undervalued (Annex III). The equilibrium real effective exchange rate approach (EREER) suggests that movements in the terms of trade are a major driver of the Real Effective Exchange Rate (REER). ${ }^{11} 12$ The

\footnotetext{
${ }^{11}$ This is based on the commodity currency model given coffee accounts for about 85 percent of exports (Cashin et al. 2002, IMF WP 02/223).

12 Only one of the three CGER methodologies was used for Burundi's external assessment due to data shortcomings.
}

undervaluation was estimated to be about 8 percent and might be explained in part by the strong increase coffee prices in 2011.

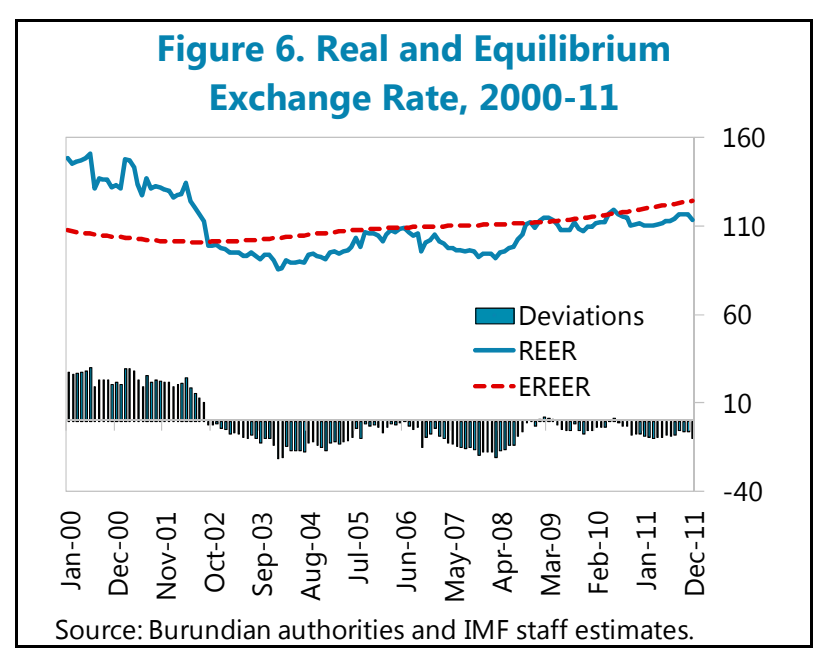

41. Burundi's reserves coverage remains appropriate to absorb external shocks.

Although gross reserve coverage in months of imports has declined recently, it remains close to 4 months, proving a buffer to smooth consumption and partially absorb external shocks. ${ }^{13}$

\footnotetext{
${ }^{13}$ Crispolti and Tsibouris (IMF WP 12/7) finds that lowincome countries with reserve cover more than three months of imports were better able to absorb external shocks relative to those with lower coverage.
} 


\section{Non-price indicators suggest room}

\section{for further improvement in}

competitiveness. Despite progress in

improving its business environment, Burundi ranked $169^{\text {th }}$ (out of 183 countries). Limited access to finance, the high cost of transportation, and poor electricity supply are cited by firms as major impediments to doing business.

\section{Burundi needs to improve its} competitiveness. Persistent electricity shortages, high transportation costs, and a difficult business environment represent key structural factors that impinge on Burundi's competitiveness. Transportation costs, which are the highest in the EAC, reflect the geographic characteristic of being landlocked. Moreover, relative productivity has consistently declined over the last decade as large aid inflows have been spent and absorbed. ${ }^{14}$

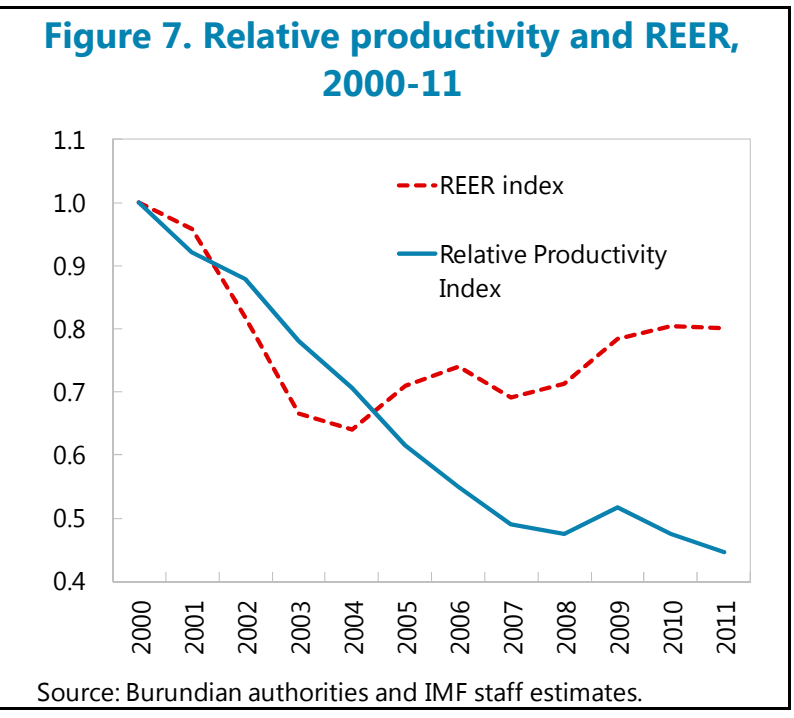

\section{Authorities' Views}

44. The authorities view the level of the exchange rate as appropriate, as the recent depreciation against the dollar by a cumulative 111/2 percent between September 2011 and May 2012 has helped improve its competitiveness.

\footnotetext{
${ }^{14}$ Relative productivity is defined as the ratio of Burundi's real per capita GDP to that of its main trading partners.
} 


\section{PROGRAM ISSUES AND RISKS}

45. It is proposed that targets for the next review are modified. The authorities met all the March 2012 targets. However, the disbursement of the World Bank budget support operation in early 2012 has lowered financing requirements of the government for the year. The new targets take into account changes in the macroeconomic outlook. The direction of macroeconomic policies under the program has not changed. The PCs and indicative targets are defined in the Technical Memorandum of Understanding (TMU).

\section{Burundi's capacity to repay the Fund} is adequate. Burundi's risk of debt distress is assessed to be high due to its narrow export base. Outstanding and prospective obligations to the Fund, measured in relation to official reserves or exports of goods and services, do not pose apparent solvency or liquidity risks (Table 6).

\section{The low statistical capacity of the}

\section{Burundian government limits the} authorities' ability to develop sound economic and social policies (MEFP \32『33). Shortcomings in the production of national accounts, weaknesses in the compilation of balance of payments, and an outdated basket used to calculate the consumer price index limits the government's ability to design and implement appropriate policies. The authorities plan to strengthen the production and dissemination of statistics, in particular those related to national accounts and consumer's price index.

\section{An update safeguards assessment of} the Banque de la République du Burundi (BRB), in the context of the new ECF arrangement, has been completed. The assessment identified some vulnerabilities that continue to represent high safeguards risks at the BRB. Governance and autonomy weaknesses need to be addressed through the strengthening of Board and Audit Committee oversight particularly in the areas of foreign reserves management and audit mechanisms respectively. Special audits of government disbursements processed through the BRB will also need to continue until longer-term mechanisms have been established to strengthen government expenditure controls. The authorities are committed to address safeguards recommendations and have already initiated steps in this regard.

\section{The success of the proposed} program under an ECF is subject to several internal and external risks. Internal risks include the potential slippages in the security situation, social unrest, and on the 
government's ability to implement program

reforms. External risks emanate from a

worsening of the global environment,

including the uncertainty in donor flows and

supply shocks that could lead to higher oil

prices. In the event of shortfalls in budget

\section{STAFF APPRAISAL}

50. In a difficult post conflict environment, Burundi has made steady, though uneven, progress in implementing its ECF-supported economic reforms. Past revenue reforms have been successful. However, reforms in other areas have been generally slow, because of limited institutional capacity.

\section{Continued implementation of} revenue reforms is critical to the program's success. Declining budget support underscores the need to increasingly finance the budget with domestic resources and to better align spending priorities in view of resource constraints.

\section{Rapid implementation of PFM} reforms would foster greater transparency and accountability of public finances and encourage donor support. Improving governance and transparency have become important aspects of donor conditionality. Staff urged the authorities to approve the new support or slippages in expenditure, further fiscal consolidation and a reprioritization of spending would be needed in order not to worsen debt sustainability.

PFM strategy which would build on the recent evaluation under the PEFA framework.

\section{Staff urged the authorities to} continue to strengthen debt management. Debt sustainability remains the anchor underpinning medium-term fiscal policy. Given the high risk of debt distress and low institutional capacity, the budget should continue to rely on grants and highly concessional external financing. Staff encourages the authorities to regularize meetings of the central bank and ministry of finance committee to enhance coordination in executing the treasury cash flow plan. Implementation of a debt management strategy arising from the DEMPA evaluation will lay the foundation for this reform.

\section{Monetary policy should be} tightened further if inflationary pressures do not ease during the remainder of the year. Staff urged the authorities to remain vigilant in case inflationary pressures persist 
and be prepared to implement additional measures to manage inflation expectations.

\section{The authorities should continue to} closely monitor risks to the financial sector.

Risks are accentuated by the unfavorable external environment, persistence of shocks and tighter liquidity conditions. Staff encourages the authorities to step-up on site inspections in order to better assess risks to the banking system.

\section{An assessment of the level of the} exchange rate suggests that the Burundian

Franc is undervalued. However other

indicators suggest further reforms are needed to improve the business climate, enhance electricity supply, and reduce cross-border transportation costs.

\section{Data provided to the Fund has} improved, but a number of shortcomings persist. Important gaps arise in the national accounts, balance of payments and consumer's prices. Priority should be given to improving statistics in these areas. Burundi continues to benefit from TA to strengthen its compilation of balance of payments, government financial statistics and consumer prices.

\section{Staff recommends completion of the} first review under the ECF arrangement based on Burundi's performance and the authorities' ownership of the program and modification of PCs for end-September 2012.

59. It is proposed that the next Article IV consultation takes place within 24 months, subject to the decision on consultation cycles (Decision No. 14747- (10/96), September 28, 2010). 
Figure 8. Burundi: Macroeconomic Developments, 2007-2012

Growth has been volatile and remains below the regional average.

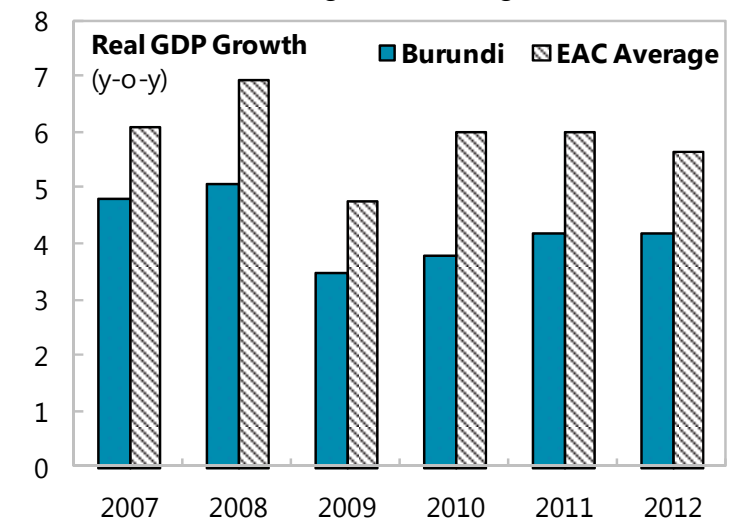

The substantial rise in revenue cannot offset the shortfall in budget support in 2012...

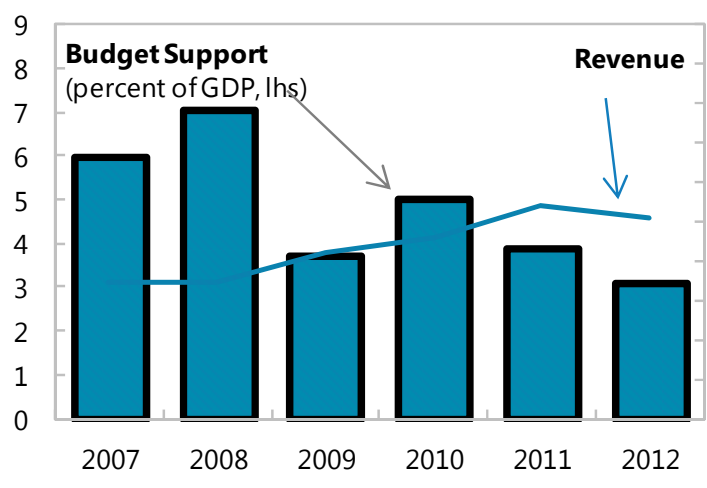

The current account remains elevated in part due

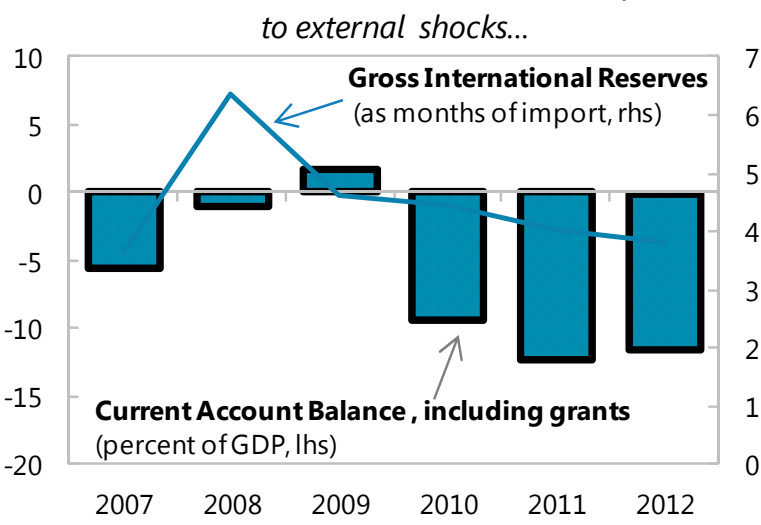

Second round effects are becoming entrenched.

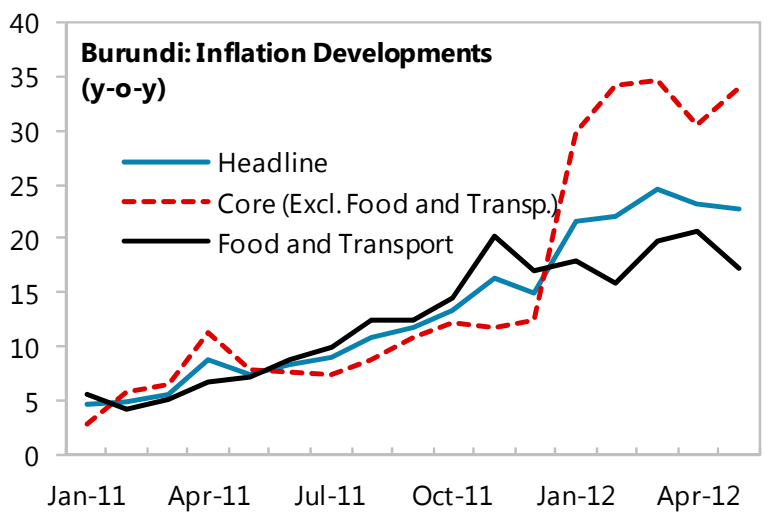

...while domestic spending needs to be pruned given lower financing.

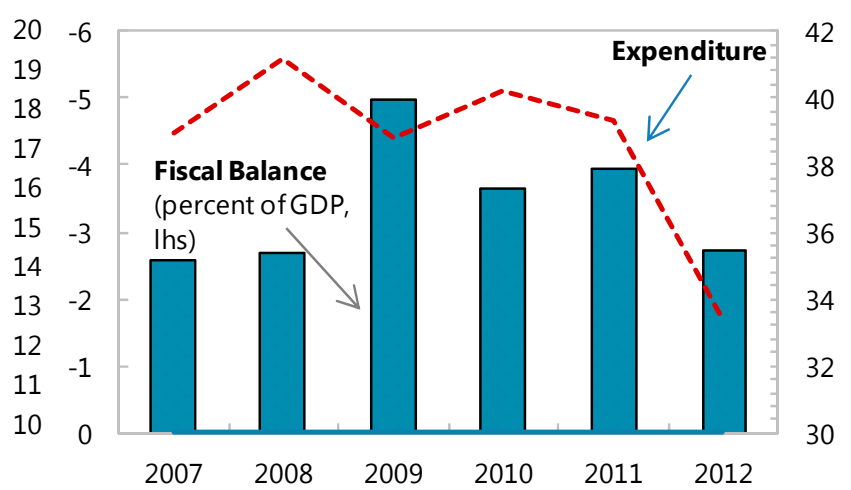

.... while the REER appreciated reflecting higher inflation than in trading partners.

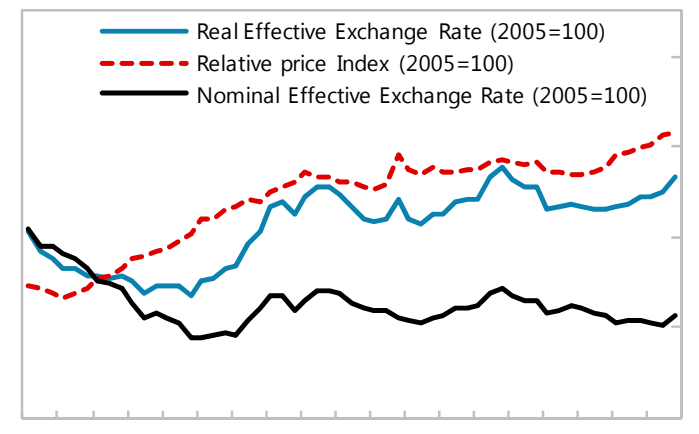

Jan-07 Oct-07 Jul-08 Apr-09 Jan-10 Oct-10 Jul-11

Source: Burundi authortities and IMF staff estimates and projections. 
Figure 9. Burundi: Monetary Developments, 2008-2012

Credit growth to the private sector has slowed down after growing strongly ...

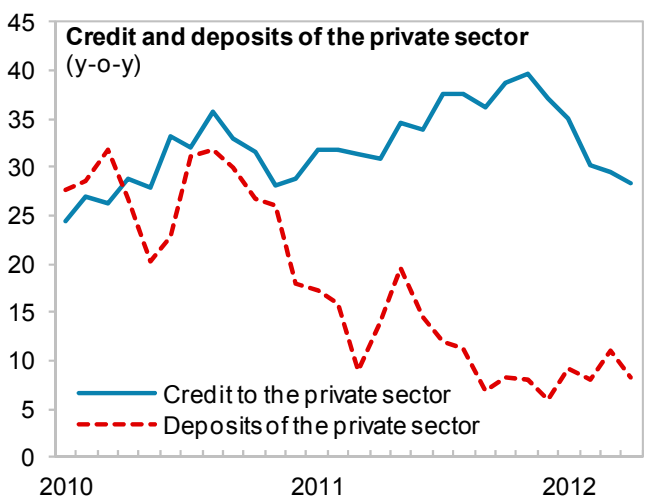

More recently, monetary aggregates have slowed down ...

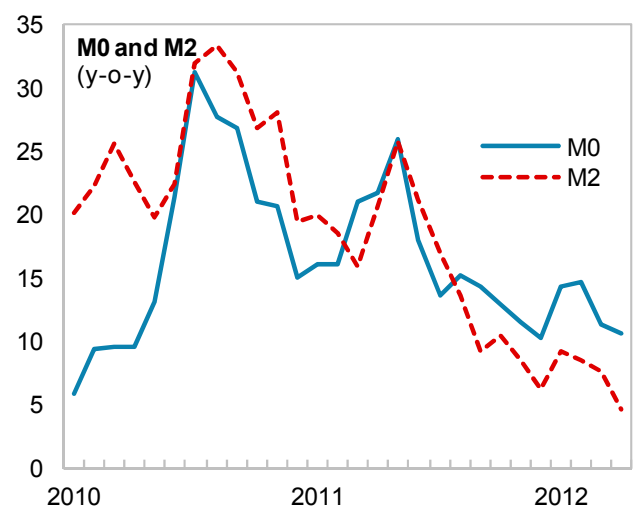

Policy rates rising in line with regional trends...

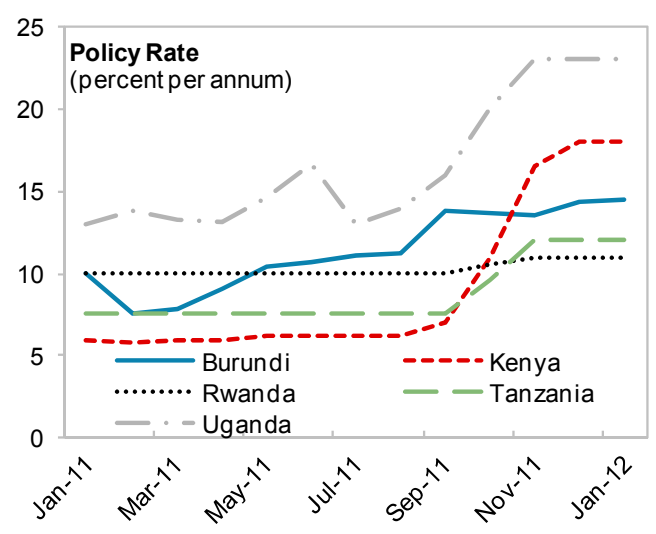

... albeit from a low base.

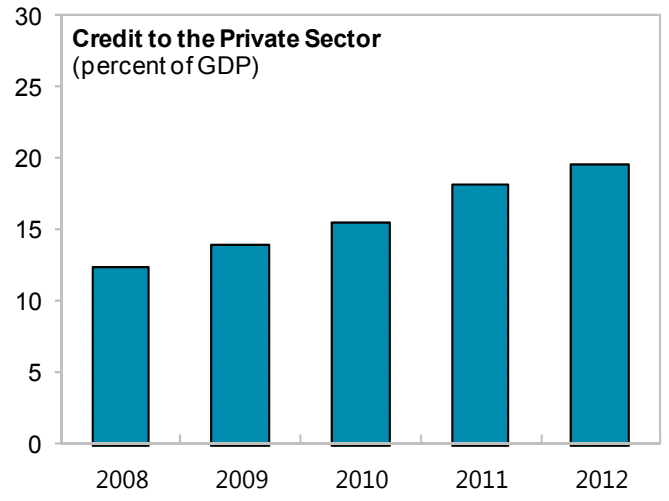

... as interest rates increased and liquidity tightened.

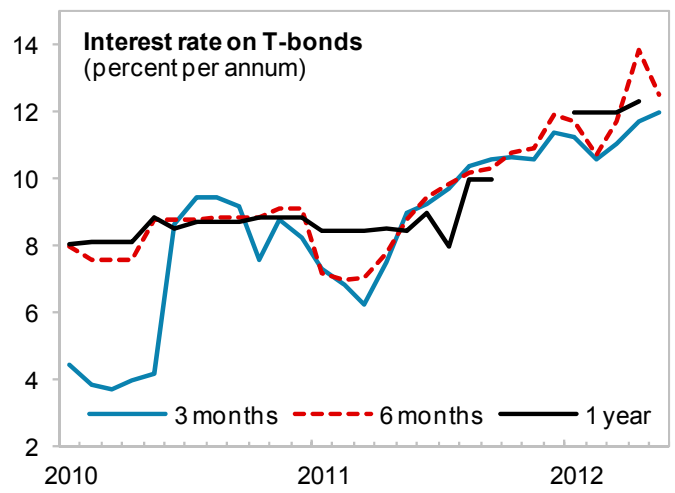

...to contain inflationary pressures.

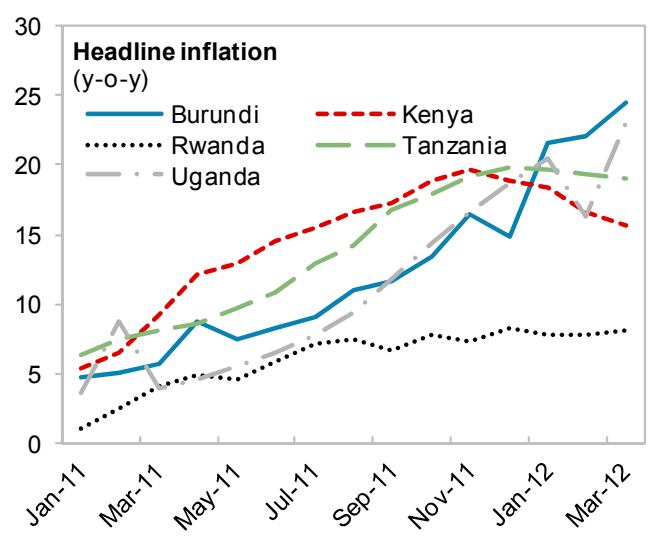

Source: Burundi authorities and IMF staff estimates and projections. 


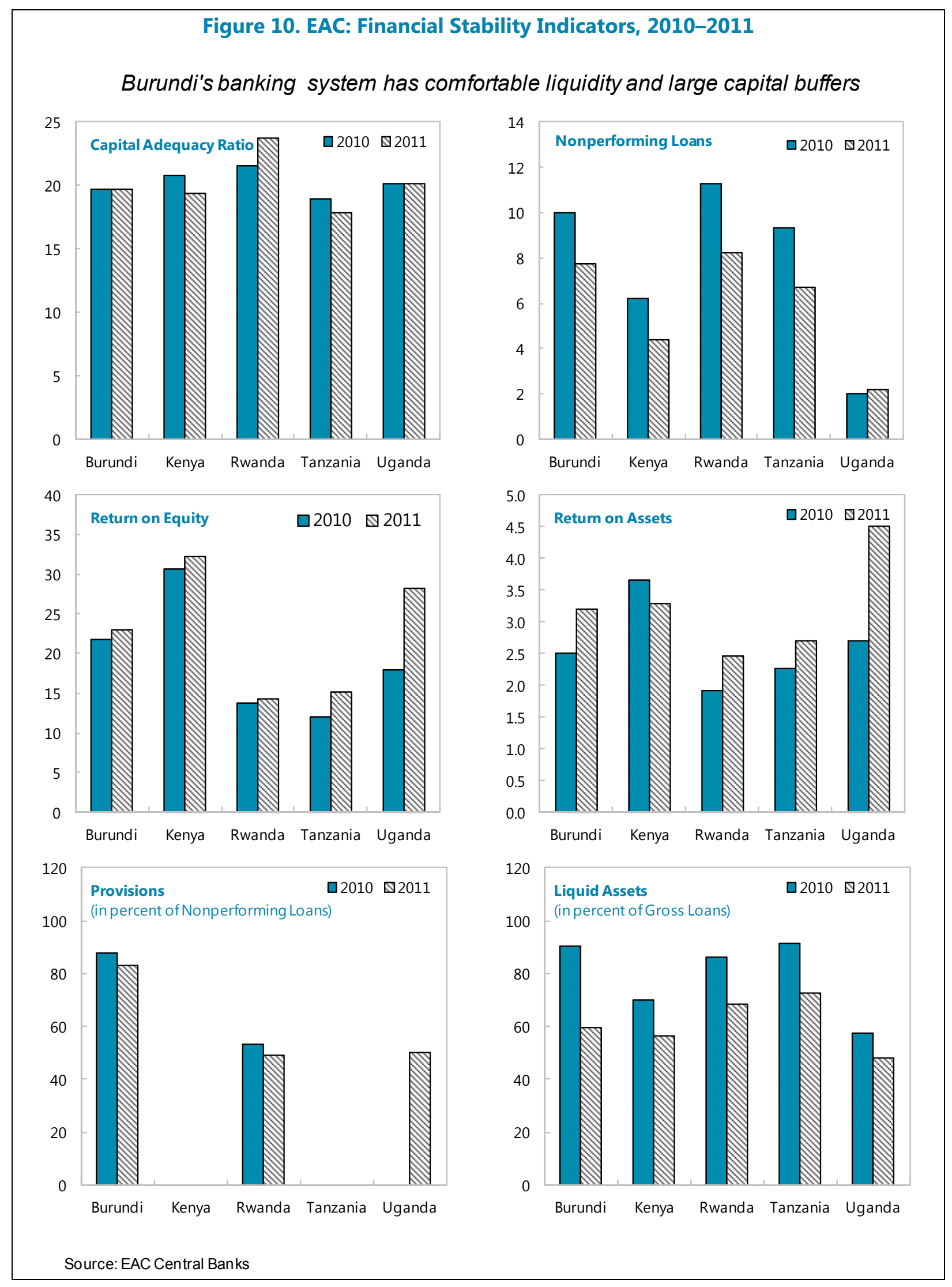




\begin{tabular}{|c|c|c|c|c|c|c|c|c|}
\hline & \multirow[t]{2}{*}{2010} & \multicolumn{2}{|c|}{2011} & \multicolumn{2}{|c|}{2012} & \multirow{2}{*}{2013} & \multirow{2}{*}{$\begin{array}{l}2014 \\
\text { Proj. }\end{array}$} & \multirow[t]{2}{*}{2015} \\
\hline & & Prog. ${ }^{1}$ & Prel. & Prog. ${ }^{1}$ & Proj. & & & \\
\hline & \multicolumn{8}{|c|}{ (Annual percentage change) } \\
\hline \multicolumn{9}{|l|}{ National income and prices } \\
\hline Real GDP growth & 3.8 & 4.2 & 4.2 & 4.8 & 4.2 & 4.5 & 5.1 & 5.5 \\
\hline GDP deflator & 12.3 & 13.1 & 14.3 & 9.9 & 15.2 & 11.1 & 7.2 & 5.8 \\
\hline Consumer prices (period average) & 6.4 & 9.5 & 9.7 & 11.7 & 19.6 & 6.4 & 7.4 & 6.3 \\
\hline Consumer prices (end of period) & 4.1 & 14.3 & 14.9 & 10.3 & 14.7 & 8.4 & 6.1 & 6.4 \\
\hline \multicolumn{9}{|l|}{ External sector } \\
\hline Exports, f.o.b. (US\$) & 48.0 & 1.4 & 22.5 & 20.4 & 3.1 & 0.2 & -1.0 & 9.3 \\
\hline Imports, f.o.b. (US\$) & 105.3 & 6.7 & 3.7 & 0.2 & -2.9 & 3.5 & 1.2 & 3.7 \\
\hline Export volume & 16.4 & -21.9 & 0.4 & 27.8 & 30.4 & 5.4 & -3.9 & 14.8 \\
\hline Import volume & 145.1 & 1.3 & -22.6 & 3.7 & 0.8 & 7.9 & 2.8 & 4.6 \\
\hline \multirow[t]{2}{*}{ Terms of trade (deterioration $=-$ ) } & 51.7 & 4.6 & -8.9 & -2.5 & -17.9 & -1.0 & 4.6 & -4.0 \\
\hline & \multicolumn{8}{|c|}{$\begin{array}{c}\text { (Change in percent of beginning of period M2, } \\
\text { unless otherwise indicated) }\end{array}$} \\
\hline \multicolumn{9}{|l|}{ Money and credit } \\
\hline Net foreign assets & -5.4 & -2.6 & -12.1 & 1.0 & -0.6 & 6.1 & -0.8 & 7.6 \\
\hline Domestic credit & 25.1 & 21.3 & 30.7 & 16.1 & 28.6 & 14.6 & 23.3 & 11.4 \\
\hline Government & 7.4 & 3.9 & 8.0 & 8.1 & 5.4 & 2.6 & 2.2 & 2.2 \\
\hline Private sector & 17.0 & 17.4 & 24.1 & 7.9 & 23.3 & 12.5 & 21.1 & 9.1 \\
\hline Money and quasi-money (M2) & 19.4 & 15.8 & 6.1 & 13.1 & 18.4 & 16.1 & 12.7 & 11.6 \\
\hline Reserve money (12-month growth rate) & 5.7 & 13.5 & 0.6 & 12.0 & 28.1 & 9.3 & 16.4 & 13.3 \\
\hline & \multicolumn{8}{|c|}{ (Percent of GDP) } \\
\hline \multicolumn{9}{|l|}{ General government } \\
\hline Revenue and grants & 37.3 & 37.9 & 36.1 & 32.1 & 30.8 & 30.4 & 29.9 & 28.6 \\
\hline Tax and nontax revenue & 14.6 & 15.5 & 15.4 & 16.2 & 15.1 & 15.5 & 15.6 & 15.6 \\
\hline Total expenditure & 41.0 & 40.4 & 40.0 & 35.6 & 33.6 & 34.9 & 34.8 & 32.5 \\
\hline Net lending $(+) /$ borrowing $(-)$ & -3.6 & -2.5 & -4.0 & -3.5 & -2.7 & -4.6 & -4.9 & -3.9 \\
\hline \multicolumn{9}{|l|}{ External sector } \\
\hline Current account balance & -9.4 & -12.5 & -12.3 & -12.0 & -11.6 & -11.0 & -10.8 & -11.1 \\
\hline Overall balance of payments & 0.7 & -0.8 & -1.6 & -0.2 & -0.3 & -0.3 & -2.2 & -0.9 \\
\hline Savings-investment balance & -9.4 & -12.5 & -12.3 & -12.0 & -11.6 & -11.0 & -10.8 & -11.1 \\
\hline Private & -5.8 & -9.9 & -8.3 & -8.5 & -8.8 & -6.5 & -5.9 & -7.2 \\
\hline Public & -3.6 & -2.5 & -4.0 & -3.5 & -2.7 & -4.6 & -4.9 & -3.9 \\
\hline \multicolumn{9}{|l|}{ External sector } \\
\hline Gross official reserves (US\$ million) & 332 & 314 & 296 & 308 & 288 & 319 & 308 & 342 \\
\hline Months of imports & 4.4 & 4.4 & 4.0 & 4.4 & 3.8 & 4.2 & 3.9 & 4.7 \\
\hline Debt-service to exports ratio (percent) & 1.4 & 4.2 & 3.3 & 5.8 & 3.8 & 7.2 & 10.6 & 13.1 \\
\hline \multicolumn{9}{|l|}{ Memorandum item: } \\
\hline Nominal Exchange rate (BIF/USD) & 1231 & $\ldots$ & 1261 & $\ldots$ & $\ldots$ & $\ldots$ & $\ldots$ & $\ldots$ \\
\hline GDP at current market prices (BIF billion) & 2495 & 2933 & 2971 & 3367 & 3566 & 4138 & 4663 & 5203 \\
\hline Nominal GDP per Capita (US Dollars) & 242 & 269 & 255 & 291 & 289 & 311 & 332 & 349 \\
\hline Sources: Burundi authorities; IMF staff estimates an & ns. & & & & & & & \\
\hline${ }^{1}$ IMF Country Report 12/28. & & & & & & & & \\
\hline
\end{tabular}




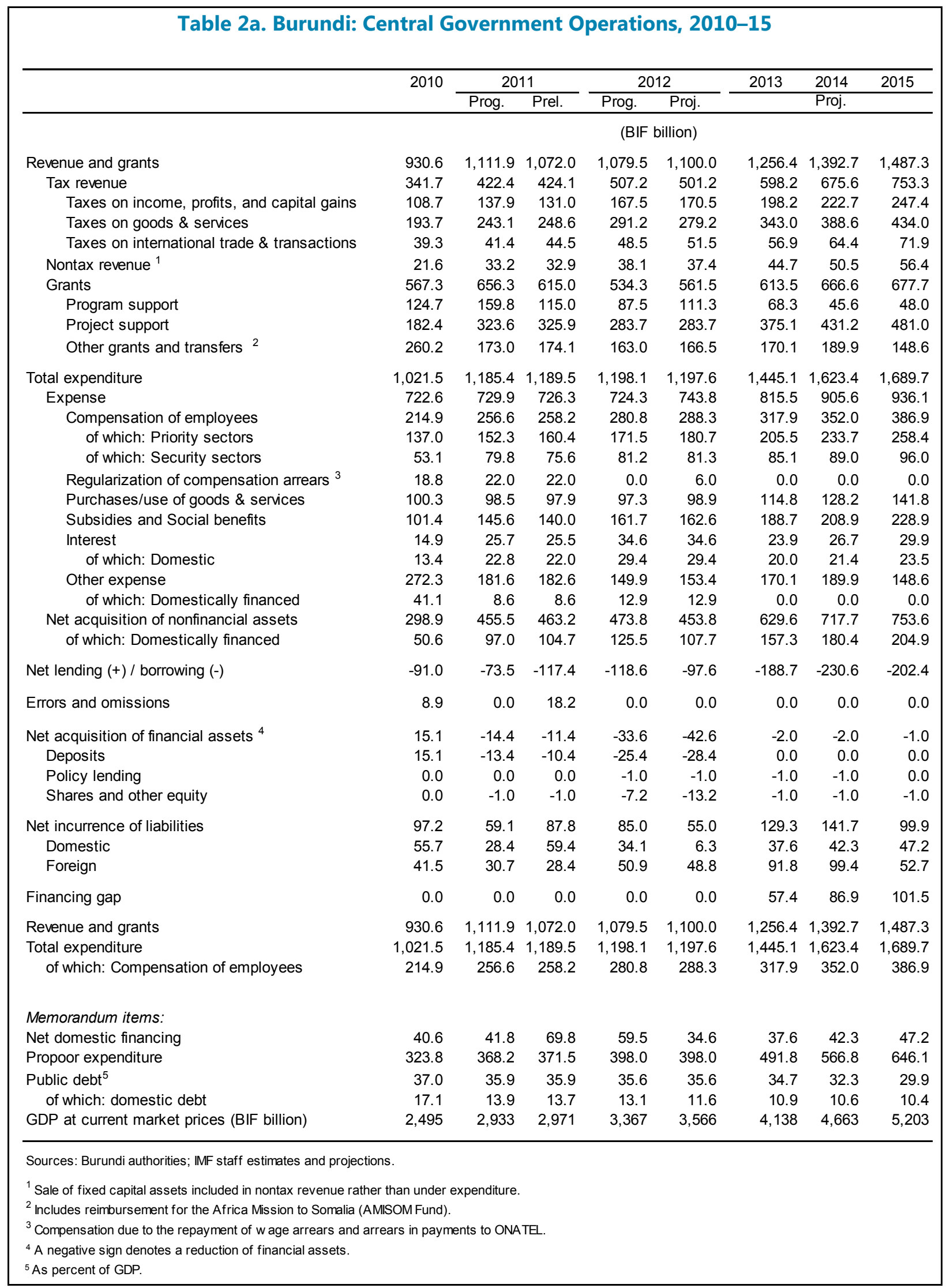




\begin{tabular}{|c|c|c|c|c|c|c|c|c|}
\hline \multicolumn{9}{|c|}{ Table 2b. Burundi: Central Government Operations, 2010-15 } \\
\hline & \multirow[t]{2}{*}{2010} & \multicolumn{2}{|c|}{2011} & \multicolumn{2}{|c|}{2012} & \multirow[t]{2}{*}{2013} & \multirow{2}{*}{\begin{tabular}{|l|}
2014 \\
Proj. \\
\end{tabular}} & \multirow[t]{2}{*}{2015} \\
\hline & & Prog. & Prel. & Prog. & Proj. & & & \\
\hline & \multicolumn{8}{|c|}{ (Percent of GDP, unless otherwise indicated) } \\
\hline Revenue and grants & 37.3 & 37.9 & 36.1 & 32.1 & 30.8 & 30.4 & 29.9 & 28.6 \\
\hline Tax revenue & 13.7 & 14.4 & 14.3 & 15.1 & 14.1 & 14.5 & 14.5 & 14.5 \\
\hline Taxes on income, profits, and capital gains & 4.4 & 4.7 & 4.4 & 5.0 & 4.8 & 4.8 & 4.8 & 4.8 \\
\hline Taxes on goods \& services & 7.8 & 8.3 & 8.4 & 8.6 & 7.8 & 8.3 & 8.3 & 8.3 \\
\hline Taxes on international trade $\&$ transactions & 1.6 & 1.4 & 1.5 & 1.4 & 1.4 & 1.4 & 1.4 & 1.4 \\
\hline Nontax revenue ${ }^{1}$ & 0.9 & 1.1 & 1.1 & 1.1 & 1.0 & 1.1 & 1.1 & 1.1 \\
\hline Grants & 22.7 & 22.4 & 20.7 & 15.9 & 15.7 & 14.8 & 14.3 & 13.0 \\
\hline Program support & 5.0 & 5.4 & 3.9 & 2.6 & 3.1 & 1.7 & 1.0 & 0.9 \\
\hline Project support & 7.3 & 11.0 & 11.0 & 8.4 & 8.0 & 9.1 & 9.2 & 9.2 \\
\hline Other grants and transfers ${ }^{2}$ & 10.4 & 5.9 & 5.9 & 4.8 & 4.7 & 4.1 & 4.1 & 2.9 \\
\hline Total expenditure & 41.0 & 40.4 & 40.0 & 35.6 & 33.6 & 34.9 & 34.8 & 32.5 \\
\hline Expense & 29.0 & 24.9 & 24.4 & 21.5 & 20.9 & 19.7 & 19.4 & 18.0 \\
\hline Compensation of employees & 8.6 & 8.7 & 8.7 & 8.3 & 8.1 & 7.7 & 7.5 & 7.4 \\
\hline of which: Priority sectors & 5.5 & 5.2 & 5.4 & 5.1 & 5.1 & 5.0 & 5.0 & 5.0 \\
\hline of which: Security sectors & 2.1 & 2.7 & 2.5 & 2.4 & 2.3 & 2.1 & 1.9 & 1.8 \\
\hline Regularization of compensation arrears ${ }^{3}$ & 0.8 & 0.8 & 0.7 & 0.0 & 0.2 & 0.0 & 0.0 & 0.0 \\
\hline Purchases/use of goods \& services & 4.0 & 3.4 & 3.3 & 2.9 & 2.8 & 2.8 & 2.7 & 2.7 \\
\hline Subsidies and Social benefits & 4.1 & 5.0 & 4.7 & 4.8 & 4.6 & 4.6 & 4.5 & 4.4 \\
\hline Interest & 0.6 & 0.9 & 0.9 & 1.0 & 1.0 & 0.6 & 0.6 & 0.6 \\
\hline of which: Domestic & 0.5 & 0.8 & 0.7 & 0.9 & 0.8 & 0.5 & 0.5 & 0.5 \\
\hline Other expense & 10.9 & 6.2 & 6.1 & 4.5 & 4.3 & 4.1 & 4.1 & 2.9 \\
\hline of which: Domestically financed & 1.6 & 0.3 & 0.3 & 0.4 & 0.4 & 0.0 & 0.0 & 0.0 \\
\hline Net acquisition of nonfinancial assets & 12.0 & 15.5 & 15.6 & 14.1 & 12.7 & 15.2 & 15.4 & 14.5 \\
\hline of which: Domestically financed & 2.0 & 3.3 & 3.5 & 3.7 & 3.0 & 3.8 & 3.9 & 3.9 \\
\hline Net lending $(+) /$ borrowing (-) & -3.6 & -2.5 & -4.0 & -3.5 & -2.7 & -4.6 & -4.9 & -3.9 \\
\hline Errors and ommisions & 0.4 & 0.0 & 0.6 & 0.0 & 0.0 & 0.0 & 0.0 & 0.0 \\
\hline Net acquisition of financial assets ${ }^{4}$ & 0.6 & -0.5 & -0.4 & -1.0 & -1.2 & 0.0 & 0.0 & 0.0 \\
\hline Deposits & 0.6 & -0.5 & -0.4 & -0.8 & -0.8 & 0.0 & 0.0 & 0.0 \\
\hline Policy lending & 0.0 & 0.0 & 0.0 & 0.0 & 0.0 & 0.0 & 0.0 & 0.0 \\
\hline Shares and other equity & 0.0 & 0.0 & 0.0 & -0.2 & -0.4 & 0.0 & 0.0 & 0.0 \\
\hline Net incurrence of liabilities & 3.9 & 2.0 & 3.0 & 2.5 & 1.5 & 3.1 & 3.0 & 1.9 \\
\hline Domestic & 2.2 & 1.0 & 2.0 & 1.0 & 0.2 & 0.9 & 0.9 & 0.9 \\
\hline Foreign & 1.7 & 1.0 & 1.0 & 1.5 & 1.4 & 2.2 & 2.1 & 1.0 \\
\hline Financing gap & 0.0 & 0.0 & 0.0 & 0.0 & 0.0 & 1.4 & 1.9 & 2.0 \\
\hline \multicolumn{9}{|l|}{ Memorandum items: } \\
\hline Net domestic financing & 1.6 & 1.4 & 2.4 & 1.8 & 1.0 & 0.9 & 0.9 & 0.9 \\
\hline Propoor expenditure & 13.0 & 12.6 & 12.5 & 11.8 & 11.2 & 11.9 & 12.2 & 12.4 \\
\hline Public debt & 37.0 & 35.9 & 35.9 & 35.6 & 35.6 & 34.7 & 32.3 & 29.9 \\
\hline of which: domestic debt & 17.1 & 13.9 & 13.7 & 13.1 & 11.6 & 10.9 & 10.6 & 10.4 \\
\hline GDP at current market prices (BIF billion) & 2,495 & 2,933 & 2,971 & 3,367 & 3,566 & 4,138 & 4,663 & 5,203 \\
\hline \multicolumn{9}{|c|}{ Sources: Burundi authorities; IMF staff estimates and projections. } \\
\hline \multicolumn{9}{|c|}{${ }^{1}$ Sale of fixed capital assets included in nontax revenue rather than under expenditure. } \\
\hline \multicolumn{9}{|c|}{${ }^{2}$ Includes reimbursement for the Africa Mission to Somalia (AMISOM Fund). } \\
\hline \multicolumn{9}{|c|}{${ }^{3}$ Compensation due to the repayment of wage arrears and arrears in payments to ONATEL. } \\
\hline${ }^{4} \mathrm{~A}$ negative sign denotes a reduction of financial assets. & & & & & & & & \\
\hline
\end{tabular}


Table 3. Burundi: Monetary Survey, 2010-13

\begin{tabular}{|c|c|c|c|c|c|c|}
\hline & \multirow[t]{2}{*}{2010} & \multicolumn{2}{|c|}{2011} & \multicolumn{2}{|c|}{2012} & \multirow[t]{2}{*}{2013} \\
\hline & & Prog. ${ }^{1}$ & Prel. & Prog. ${ }^{1}$ & Proj. & \\
\hline & \multicolumn{5}{|c|}{ (BIF billion) } & \\
\hline Net foreign assets & 139.9 & 123.7 & 63.3 & 131.3 & 59.3 & 107.9 \\
\hline Central bank & 104.3 & 80.0 & 26.2 & 79.6 & 14.1 & 52.8 \\
\hline Deposit money banks & 35.7 & 43.7 & 37.1 & 51.7 & 45.1 & 55.1 \\
\hline Net domestic assets & 644.9 & 790.5 & 771.1 & 908.3 & 971.2 & 1087.9 \\
\hline Domestic credit & 692.4 & 827.3 & 887.2 & 945.1 & 1079.7 & 1196.3 \\
\hline Net claims on the government & 291.9 & 316.5 & 342.4 & 376.0 & 378.4 & 399.4 \\
\hline Of which: on the treasury & 303.1 & 327.8 & 355.9 & 387.3 & 390.6 & 411.5 \\
\hline Credit to the economy & 400.5 & 510.8 & 544.8 & 569.1 & 701.3 & 796.9 \\
\hline Other items, net (assets $=+$ ) & -47.5 & -36.7 & -116.1 & -36.7 & -108.5 & -108.4 \\
\hline M3 & 784.8 & 914.2 & 834.4 & 1039.6 & 1030.5 & 1195.9 \\
\hline Foreign currency deposits & 151.4 & 180.5 & 162.2 & 209.9 & 234.5 & 272.1 \\
\hline M2 & 633.5 & 733.7 & 672.1 & 829.7 & 796.0 & 923.8 \\
\hline Currency in circulation & 139.1 & 162.0 & 153.2 & 184.3 & 189.2 & 219.7 \\
\hline Local currency deposits & 494.4 & 571.7 & 518.9 & 645.4 & 606.8 & 704.1 \\
\hline Demand deposits & 341.3 & 392.2 & 331.8 & 439.7 & 393.4 & 456.5 \\
\hline \multirow[t]{2}{*}{ Quasi-money } & 153.1 & 179.4 & 187.1 & 205.7 & 213.4 & 247.7 \\
\hline & \multicolumn{5}{|c|}{ Change as a percentage of beginning period $\mathrm{M} 2$} & \\
\hline Net foreign assets & -5.4 & -2.6 & -12.1 & 1.0 & -0.6 & 6.1 \\
\hline Central bank & -2.7 & -3.8 & -12.3 & -0.1 & -1.8 & 4.9 \\
\hline Deposit money banks & -2.7 & 1.3 & 0.2 & 1.1 & 1.2 & 1.3 \\
\hline Net domestic assets & 27.2 & 23.0 & 19.9 & 16.1 & 29.8 & 14.7 \\
\hline Domestic credit & 25.1 & 21.3 & 30.7 & 16.1 & 28.6 & 14.6 \\
\hline Net claims on the government & 7.4 & 3.9 & 8.0 & 8.1 & 5.4 & 2.6 \\
\hline Credit to the economy & 17.7 & 17.4 & 22.8 & 7.9 & 23.3 & 12.0 \\
\hline Of which: private sector & 17.0 & 17.4 & 24.1 & 7.9 & 23.3 & 12.5 \\
\hline M3 & 21.8 & 20.4 & 7.8 & 17.1 & 29.2 & 20.8 \\
\hline Foreign currency deposits & 2.5 & 4.6 & 1.7 & 4.0 & 10.7 & 4.7 \\
\hline M2 & 19.4 & 15.8 & 6.1 & 13.1 & 18.4 & 16.1 \\
\hline Currency in circulation & 3.4 & 3.6 & 2.2 & 3.0 & 5.4 & 3.8 \\
\hline Local currency deposits & 15.9 & 12.2 & 3.9 & 10.0 & 13.1 & 12.2 \\
\hline Demand deposits & 10.9 & 8.0 & -1.5 & 6.5 & 9.2 & 7.9 \\
\hline Quasi-money & 5.0 & 4.2 & 5.4 & 3.6 & 3.9 & 4.3 \\
\hline \multicolumn{7}{|l|}{ Memorandum items: } \\
\hline Reserve money (12-month percent change) & 5.7 & 13.5 & 0.6 & 12.0 & 28.1 & 9.3 \\
\hline Velocity (GDP/M2; end of period) & 3.9 & 4.0 & 4.4 & 4.1 & 4.5 & 4.5 \\
\hline
\end{tabular}




\begin{tabular}{|c|c|c|c|c|c|c|c|c|c|c|c|c|}
\hline & \multicolumn{4}{|c|}{2010} & \multicolumn{4}{|c|}{2011} & \multicolumn{4}{|c|}{2012} \\
\hline & \multirow[t]{2}{*}{ Mar. } & \multirow[t]{2}{*}{ June } & \multirow[t]{2}{*}{ Sep. } & \multirow[t]{2}{*}{ Dec. } & \multirow[t]{2}{*}{ Mar. } & \multirow[t]{2}{*}{ June } & \multirow[t]{2}{*}{ Sep. } & \multirow[t]{2}{*}{ Dec. } & \multirow[t]{2}{*}{ Mar. } & June & Sep. & Dec. \\
\hline & & & & & & & & & & \multicolumn{3}{|c|}{ Proj. } \\
\hline & \multicolumn{12}{|c|}{ (BIF billion) } \\
\hline Net foreign assets & 105.3 & 67.7 & 44.0 & 104.3 & 107.6 & 88.8 & 40.0 & 26.2 & 16.9 & 11.4 & 15.7 & 14.1 \\
\hline Assets & 394.3 & 344.1 & 346.6 & 416.1 & 424.4 & 433.0 & 393.7 & 411.5 & 418.0 & 402.6 & 357.7 & 414.1 \\
\hline Liabilities & 289.1 & 276.3 & 302.6 & 311.8 & 316.9 & 344.3 & 353.7 & 385.4 & 401.1 & 391.1 & 342.0 & 400.0 \\
\hline Net domestic assets & 49.8 & 107.7 & 140.2 & 105.6 & 86.4 & 121.9 & 167.4 & 185.1 & 176.5 & 235.2 & 235.0 & 234.8 \\
\hline Domestic credit & 136.1 & 177.8 & 198.9 & 182.9 & 146.8 & 166.1 & 217.3 & 299.3 & 254.0 & 269.1 & 267.1 & 341.5 \\
\hline Net claims on the government & 151.8 & 171.5 & 194.7 & 183.9 & 144.5 & 158.8 & 189.7 & 266.5 & 213.7 & 239.3 & 273.5 & 265.6 \\
\hline Other credit & -15.7 & 6.3 & 4.2 & -1.0 & 2.4 & 7.2 & 27.6 & 32.8 & 40.3 & 29.8 & -6.4 & 75.9 \\
\hline Other items, net (assets $=+$ ) & -86.3 & -70.1 & -58.7 & -77.4 & -60.5 & -44.2 & -49.8 & -114.2 & -77.4 & -33.9 & -32.1 & -81.3 \\
\hline Reserve money & 155.1 & 175.4 & 184.1 & 209.8 & 193.9 & 210.6 & 207.4 & 211.2 & 193.4 & 246.6 & 250.7 & 249.0 \\
\hline Currency in circulation & 109.5 & 131.1 & 132.5 & 139.1 & 132.5 & 154.7 & 151.4 & 153.2 & 147.4 & 181.1 & 166.7 & 189.2 \\
\hline Bank reserves & 26.4 & 23.7 & 30.4 & 45.7 & 39.3 & 27.3 & 33.1 & 34.9 & 22.9 & 37.0 & 61.1 & 36.7 \\
\hline Cash in vault & 15.9 & 16.5 & 16.8 & 16.7 & 17.3 & 17.6 & 17.1 & 16.9 & 18.1 & 17.6 & 17.1 & 16.9 \\
\hline Other nonbank deposits & 3.3 & 4.1 & 4.4 & 8.3 & 4.8 & 10.9 & 5.9 & 6.2 & 5.0 & 10.9 & 5.9 & 6.2 \\
\hline \multicolumn{13}{|l|}{ Memorandum items: } \\
\hline Net foreign assets of BRB (US\$ million) & 85.6 & 55.0 & 35.7 & 84.6 & 87.0 & 71.4 & 31.4 & 19.2 & 12.1 & 8.1 & 11.1 & 10.1 \\
\hline
\end{tabular}




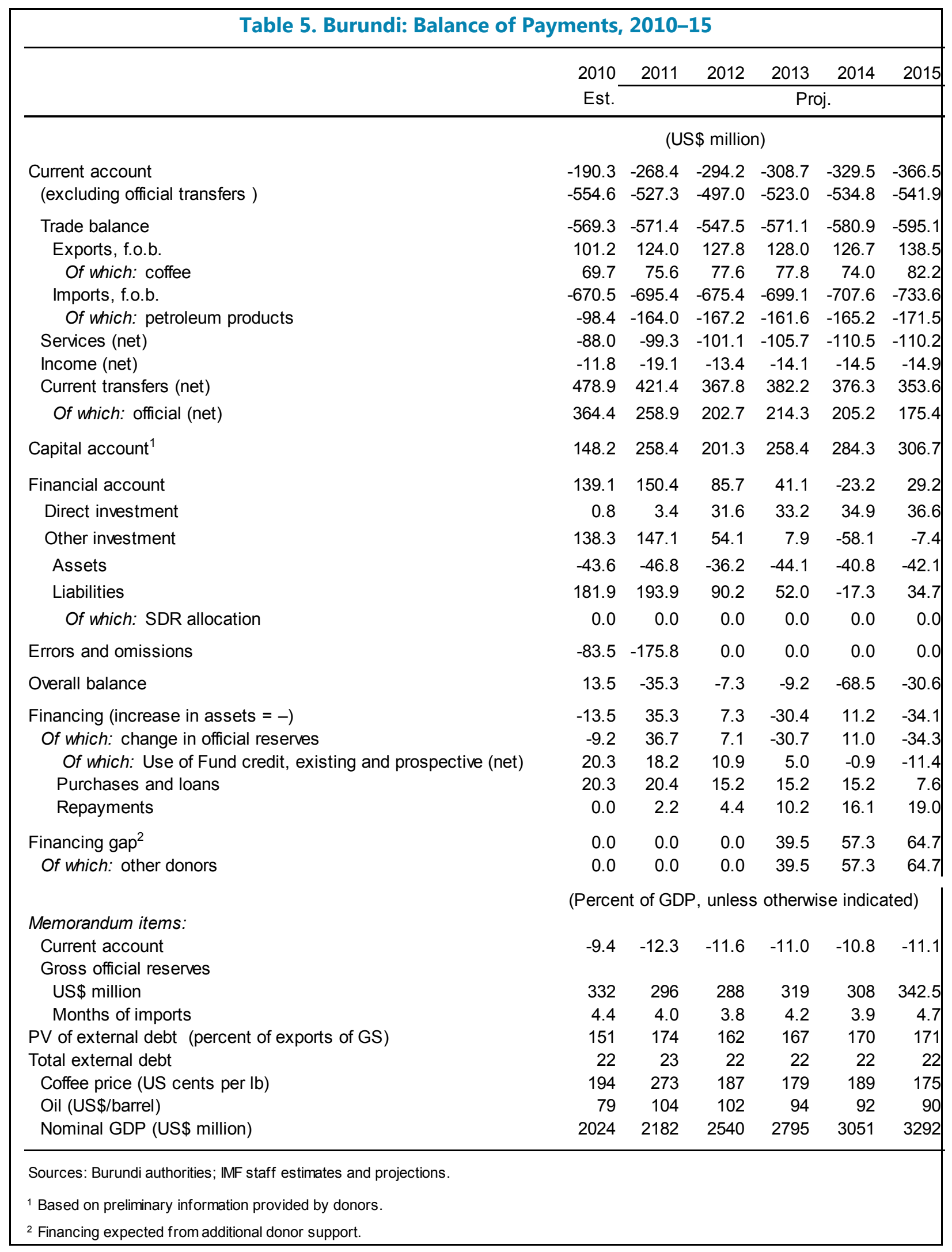


Table 6. Burundi: Indicators of Capacity to Repay the Fund, 2012-20

\begin{tabular}{|c|c|c|c|c|c|c|c|c|c|}
\hline & 2012 & 2013 & 2014 & 2015 & 2016 & 2017 & 2018 & 2019 & 2020 \\
\hline & \multicolumn{9}{|c|}{ Projections } \\
\hline \multicolumn{10}{|l|}{$\begin{array}{l}\text { Fund obligations based on existing credit } \\
\text { (SDR million) }\end{array}$} \\
\hline Principal & 2.9 & 6.7 & 10.6 & 12.5 & 13.0 & 12.0 & 11.2 & 8.5 & 5.8 \\
\hline Charges and interest & 0.0 & 0.0 & 0.2 & 0.2 & 0.1 & 0.1 & 0.1 & 0.0 & 0.0 \\
\hline \multicolumn{10}{|c|}{$\begin{array}{l}\text { Fund obligations based on existing and prospective credit } \\
\text { (SDR million) }\end{array}$} \\
\hline Principal & 2.9 & 6.7 & 10.6 & 12.5 & 13.0 & 12.0 & 12.5 & 11.8 & 11.1 \\
\hline Charges and interest & 0.0 & 0.0 & 0.2 & 0.2 & 0.2 & 0.2 & 0.1 & 0.1 & 0.1 \\
\hline \multicolumn{10}{|c|}{ Total obligations based on existing and prospective credit } \\
\hline SDR million & 2.9 & 6.7 & 10.8 & 12.7 & 13.2 & 12.2 & 12.6 & 11.9 & 11.2 \\
\hline Percent of exports of goods and services & 1.9 & 4.4 & 7.1 & 7.7 & 8.3 & 7.0 & 6.5 & 5.5 & 4.6 \\
\hline Percent of debt service $^{1}$ & 49.0 & 60.8 & 67.1 & 58.5 & 58.6 & 49.8 & 45.9 & 41.6 & 37.7 \\
\hline Percent of gross official reserves & 1.5 & 3.2 & 5.3 & 5.6 & 5.4 & 4.4 & 4.2 & 3.8 & 3.5 \\
\hline Percent of GDP & 0.2 & 0.4 & 0.5 & 0.6 & 0.6 & 0.5 & 0.4 & 0.4 & 0.3 \\
\hline Percent of quota & 3.7 & 8.7 & 14.0 & 16.5 & 17.1 & 15.8 & 16.4 & 15.4 & 14.5 \\
\hline \multicolumn{10}{|l|}{ Outstanding Fund credit } \\
\hline SDR million & 88.0 & 91.2 & 90.7 & 83.2 & 70.2 & 58.2 & 45.8 & 34.0 & 22.9 \\
\hline Percent of exports of goods and services & 57.9 & 59.5 & 59.8 & 50.1 & 44.0 & 33.5 & 23.6 & 15.7 & 9.5 \\
\hline Percent of debt service ${ }^{1}$ & $1,507.4$ & 824.3 & 563.6 & 382.6 & 312.4 & 238.6 & 166.7 & 119.2 & 77.1 \\
\hline Percent of gross official reserves & 46.7 & 43.5 & 44.8 & 36.9 & 28.5 & 21.3 & 15.2 & 11.0 & 7.1 \\
\hline Percent of GDP & 5.3 & 5.0 & 4.5 & 3.8 & 3.0 & 2.2 & 1.5 & 1.0 & 0.6 \\
\hline Percent of quota & 114.2 & 118.5 & 117.8 & 108.0 & 91.2 & 75.6 & 59.4 & 44.2 & 29.7 \\
\hline Net use of Fund credit (SDR million) & 7.1 & 3.3 & -0.6 & -7.5 & -13.0 & -12.0 & -12.5 & -11.8 & -11.1 \\
\hline Disbursements & 10.0 & 10.0 & 10.0 & 5.0 & -- & -- & -- & -- & - \\
\hline Repayments and repurchases & 2.9 & 6.7 & 10.6 & 12.5 & 13.0 & 12.0 & 12.5 & 11.8 & 11.1 \\
\hline \multicolumn{10}{|l|}{ Memorandum items: } \\
\hline Exports of goods and services (US\$ million) & 232.8 & 233.6 & 230.8 & 252.3 & 242.2 & 263.7 & 293.8 & 327.8 & 366.4 \\
\hline Debt service (US\$ million) & 8.9 & 16.9 & 24.5 & 33.1 & 34.1 & 37.0 & 41.7 & 43.3 & 45.0 \\
\hline Gross official reserves (US\$ million) & 288.5 & 319.2 & 308.2 & 342.5 & 373.7 & 414.8 & 455.5 & 468.3 & 486.9 \\
\hline Nominal GDP ((US\$ million) & $2,539.9$ & $2,795.2$ & $3,051.3$ & $3,292.3$ & 3586.4 & 4003.8 & 4487.3 & 5035.0 & 5657.2 \\
\hline Quota (SDR, million) & 77.0 & 77.0 & 77.0 & 77.0 & 77.0 & 77.0 & 77.0 & 77.0 & 77.0 \\
\hline
\end{tabular}


Table 7. Burundi: Actual and Projected Schedule of Disbursements and Reviews, 2012-15

\begin{tabular}{|c|c|c|c|}
\hline Date & $\begin{array}{l}\text { Disbursement } \\
\text { (SDR million) }\end{array}$ & $\begin{array}{l}\text { Percent } \\
\text { of quota }\end{array}$ & Conditions \\
\hline January 27, 2012 & 1 & 1.3 & Executive Board approval \\
\hline July 15, 2012 & 4 & 5.2 & $\begin{array}{l}\text { Completion of first review, based on } \\
\text { observance of performance criteria at } \\
\text { end-March 2012. }\end{array}$ \\
\hline January 15, 2013 & 5 & 6.5 & $\begin{array}{l}\text { Completion of second review, based on } \\
\text { observance of performance criteria at } \\
\text { end-September } 2012 \text {. }\end{array}$ \\
\hline July 15, 2013 & 5 & 6.5 & $\begin{array}{l}\text { Completion of third review, based on } \\
\text { observance of performance criteria at } \\
\text { end-March } 2013 \text {. }\end{array}$ \\
\hline January 15, 2014 & 5 & 6.5 & $\begin{array}{l}\text { Completion of fourth review, based on } \\
\text { observance of performance criteria at } \\
\text { end-September } 2013 \text {. }\end{array}$ \\
\hline July 15, 2014 & 5 & 6.5 & $\begin{array}{l}\text { Completion of fifth review, based on } \\
\text { observance of performance criteria at } \\
\text { end-March } 2014 \text {. }\end{array}$ \\
\hline January 15, 2015 & 5 & 6.5 & $\begin{array}{l}\text { Completion of sixth review, based on } \\
\text { observance of performance criteria at } \\
\text { end-September } 2014 \text {. }\end{array}$ \\
\hline $\begin{array}{l}\text { Total for the ECF } \\
\text { arrangement }\end{array}$ & 30 & 39 & \\
\hline
\end{tabular}




\begin{tabular}{|c|c|c|c|c|c|c|c|}
\hline \multicolumn{8}{|c|}{$\begin{array}{l}\text { Table 8. Burundi: Banking System Soundness Indicators, 2009-12 } \\
\text { (percent, unless otherwise indicated) }\end{array}$} \\
\hline & 2009 & 2010 & 2011 & 2011 & 2011 & 2011 & 2012 \\
\hline \multicolumn{8}{|l|}{ Capital Requirement } \\
\hline Capital requirement over weighted assets (solvency ratio) & 19.1 & 19.7 & 21.7 & 20.7 & 20.0 & 19.8 & 19.8 \\
\hline Core capital (Tier 1 capital) over weighted assets & 15.5 & 16.9 & 18.9 & 18.1 & 17.6 & 17.3 & 17.3 \\
\hline \multicolumn{8}{|l|}{ Quality of assets } \\
\hline Nonperforming loans (percent of total gross loans granted) & 13.0 & 10.0 & 8.8 & 7.6 & 7.3 & 7.7 & 8.1 \\
\hline Provisions (percent of nonperforming loans) & 88.7 & 87.6 & 90.5 & 90.6 & 81.9 & 83.3 & 76.7 \\
\hline Nonperforming loans net of provisions (percent of capital) & 5.4 & 4.3 & 2.8 & 2.6 & 5.0 & 4.9 & 7.1 \\
\hline Large exposures (percent of capital) & 28.2 & 28.6 & 25.8 & 27.3 & 21.7 & 23.5 & 20.8 \\
\hline \multicolumn{8}{|l|}{ Profitability rates } \\
\hline Return on assets & 2.6 & 2.5 & 1.0 & 1.9 & 2.9 & 3.2 & 0.7 \\
\hline Return on equity capital & 22.8 & 21.8 & 6.7 & 13.2 & 20.3 & 23.0 & 4.6 \\
\hline Net interest (percent of gross results) & 207.0 & 191.3 & 168.3 & 164.8 & 165.7 & 175.6 & 176.9 \\
\hline Costs excluding interest (percent of gross outturn) & 172.3 & 143.4 & 101.4 & 100.0 & 121.4 & 114.0 & 128.6 \\
\hline \multicolumn{8}{|l|}{ Liquidity } \\
\hline Liquid assets (percent of all loans granted) & 91.4 & 90.5 & 77.7 & 55.8 & 49.5 & 59.5 & 56.0 \\
\hline Liquid assets (percent of short-term commitments) & 168.8 & 153.5 & 133.0 & 91.2 & 79.4 & 93.1 & 88.6 \\
\hline
\end{tabular}




\section{ANNEX I. BURUNDI: RISK ASSESSMENT MATRIX}

\begin{tabular}{|c|c|c|}
\hline $\begin{array}{l}\text { Nature/Sources } \\
\text { of main Threats }\end{array}$ & $\begin{array}{l}\text { Likelihood of realization in the } \\
\text { Next Three Years }\end{array}$ & $\begin{array}{l}\text { Expected Impact on } \\
\text { Economy if Risk is Realized }\end{array}$ \\
\hline $\begin{array}{l}\text { A deterioration of } \\
\text { the political and } \\
\text { security situation }\end{array}$ & $\begin{array}{l}\text { Staff assessment: Medium } \\
\text { Notwithstanding recent progress in } \\
\text { nation building, the political situation } \\
\text { remains fragile. } \\
\text { The UN Security Council expressed } \\
\text { concern over alleged extra-judicial } \\
\text { killings by security forces. On March } \\
27, \text { civil society and trade unions } \\
\text { conducted a strike to protest increases } \\
\text { in electricity tariffs and fuel prices. } \\
\text { The recent surge of violence in Eastern } \\
\text { Congo has not had a significant } \\
\text { impact on the security situation in } \\
\text { Burundi. However, the government } \\
\text { has deployed a battalion at the border } \\
\text { and remains ready to react, if needed, } \\
\text { in order to prevent a further } \\
\text { deterioration on this front }\end{array}$ & $\begin{array}{l}\text { Staff Assessment: Medium } \\
\text { Will lead to: } \\
\text { 1- General social unrest } \\
\text { 2- Increase pressures on fiscal } \\
\text { expenditures (mainly military } \\
\text { spending) } \\
\text { 3- Lower growth } \\
\text { Limited resources to mitigate impact. }\end{array}$ \\
\hline $\begin{array}{l}\text { Decline in donor } \\
\text { support }\end{array}$ & $\begin{array}{l}\text { Staff assessment: Medium to High } \\
\text { While multilateral donors continue to } \\
\text { provide budget support, albeit with } \\
\text { stronger conditionality, bilateral donors } \\
\text { have become less engaged } \\
\text { Perceived lack of progress on human } \\
\text { rights, and rule of law remains a key } \\
\text { concern for donors (especially bilateral } \\
\text { donors) }\end{array}$ & $\begin{array}{l}\text { Staff Assessment: High } \\
\text { Will lead to: } \\
\text { 1- Decline in total revenues (due to } \\
\text { lower grants for budget support) } \\
\text { 2- Increase in financing needs } \\
\text { 3- Higher pressures to improve } \\
\text { revenue mobilization, administration } \\
\text { and collection } \\
\text { 4- Higher pressures to rationalize } \\
\text { and contain fiscal expenditures } \\
\text { Limited resources to mitigate impact. }\end{array}$ \\
\hline
\end{tabular}




\begin{tabular}{|c|c|c|}
\hline $\begin{array}{l}\text { Worsening in the } \\
\text { terms of trade }\end{array}$ & $\begin{array}{l}\text { Staff assessment: Medium } \\
\text { Global food prices have increased by } 8 \% \\
\text { in the last four months since December } \\
2011 \text {, and in March } 2012 \text { were only } 6 \% \\
\text { below their February } 2011 \text { historical } \\
\text { peak. } \\
\text { Coffee prices have experienced a } \\
\text { downward trend during the last } 12 \\
\text { months. Arabica prices were subject to } \\
\text { further downward corrections in March, } \\
\text { while Robusta prices } \\
\text { increased. }\end{array}$ & $\begin{array}{l}\text { Staff Assessment: Medium } \\
\text { Will lead to: } \\
\text { 1- Deterioration of the export base } \\
\text { 2- Increase in inflation (due to the } \\
\text { high share of imported goods in the } \\
\text { CPI basket) } \\
\text { 3- Lower growth } \\
\text { Limited policy instruments to } \\
\text { mitigate impact. }\end{array}$ \\
\hline Inflation risk & $\begin{array}{l}\text { Staff assessment: Medium to High } \\
\text { Data limitations pose a serious challenge } \\
\text { to assess the magnitude of the } \\
\text { appropriate policy response. } \\
\text { Second round effects due mainly to } \\
\text { transport and import costs affect core } \\
\text { inflation. High pass through from } \\
\text { international commodity prices in } \\
\text { neighboring countries have a sizeable } \\
\text { effect on import prices in Burundi. }\end{array}$ & $\begin{array}{l}\text { Staff Assessment: Medium to high } \\
\text { Will lead to: } \\
\text { 1- An increase in headline inflation } \\
\text { 2- Social unrest } \\
\text { 3- Pressures on the government (and } \\
\text { on the budget) to mitigate its impact } \\
\text { Limited policy instruments to } \\
\text { mitigate impact. }\end{array}$ \\
\hline $\begin{array}{l}\text { Strong } \\
\text { intensification of } \\
\text { the Euro Zone } \\
\text { crisis }\end{array}$ & $\begin{array}{l}\text { Staff assessment: Medium The } \\
\text { slowdown in the Euro zone, and the } \\
\text { speed of the recovery depend heavily on } \\
\text { the nature of sovereign debt } \\
\text { restructuring implementation, and } \\
\text { credibility of fiscal adjustment going } \\
\text { forward. }\end{array}$ & $\begin{array}{l}\text { Staff Assessment: Medium } \\
\text { Limited exposure from Burundi } \\
\text { banks to the Euro zone. } \\
\text { Nevertheless, Burundi would } \\
\text { experience a slowdown in } \\
\text { international trade. }\end{array}$ \\
\hline
\end{tabular}




\section{ANNEX II. BURUNDI-SHOULD THE FUEL PRICING}

\section{MECHANISM BE REVISED? ${ }^{1}$}

Burundi has been using a fuel pricing mechanism (formula) for more than two years which has been more recently complemented with a price smoothing system based on the use of a stabilization fund. In May 2011, a fuel price subsidy was introduced. This note: (i) summarizes Burundi's fuel pricing arrangement and explains some of its shortcomings; (ii) reviews the motivation for adopting an automatic fuel pricing mechanism with a price smoothing framework and highlights some design principles'; and (iii) suggests some modifications to Burundi's fuel pricing mechanism.

\section{THE CURRENT FUEL PRICING MECHANISM IN BURUNDI}

\section{Burundi's fuel pricing formula establishes a procedure to set retail prices based on the} international fuel prices faced by importers, pre-agreed distribution costs (transport, deposit and other administrative costs), pre-agreed margins for private importers and distributors, and taxes. Each month the formula stipulates the retail prices needed to ensure meeting the agreed margins, given the prices of refined fuels in international markets (obtained from Platts) and the import cost parameters included in the formula. ${ }^{3}$

\section{More recently the original formula was modified, and a retail price stabilization fund (FS) was} created. The current formula includes a lump-sum charge per liter of each fuel distributed (the amount differs by fuel product). This amount, which is charged at customs, would be transferred to the FS and deposited into a specific subaccount in the Central Bank. The purpose of the FS is to smooth fluctuations in retail prices. However, it is not an automatic scheme: the government needs to decide, each period, whether to adjust or not the contribution to the FS and, consequently, what retail price to set. Regarding tax revenue, the use of the FS should not entail any revenue losses nor

\footnotetext{
${ }^{1}$ Prepared by Bertrand Gruss and Agustin Roitman (FAD).

${ }^{2}$ This note draws heavily on the FAD technical manual "On the Design and Implementation of Automatic Fuel Pricing Mechanisms" by D. Coady, A Tuladhar, J. Arze del Granado, L. Eyraud and L. Nemeth.

${ }^{3}$ In Burundi, given that its landlocked position imply an important delay between the time fuel is purchased abroad and available for sale in the domestic market, a two-month average import price (Platts) is used.
} 
volatility in tax receipts in principle, as price smoothing would be implemented only as long as there is a positive balance in the FS. In practice, however, this is not completely true and, in any case, hard to verify as explained below.

\section{A. Shortcomings of the Current Framework: Recent Evidence}

\section{Some of the limitations are related to implementation aspects of the original framework and}

others, with the operation of the price smoothing mechanism (i.e. the FS). First, the updating of the formula's cost structure is unsatisfactory, since the different costs embedded in the pricing formula are updated with an irregular frequency. Second, the non-automatic nature of the FS has exposed the government to regular pressures on its pricing decision, similar to those experienced by countries that do not count with a formula at all. As a consequence, 3 undesirable outcomes emerge:

Large price jumps and distortions. International fuel prices fluctuations, that turn out to be very persistent, affecting import prices, are confused with transitory ones, often leading to long periods of fixed prices followed by large single price adjustments (or, a sequence of relatively large adjustments). ${ }^{4}$

Lack of fiscal transparency. Withdrawals from the FS are implemented by adjusting excise taxes. In principle this would not entail fiscal losses if there is a consequent compensation with FS resources. However, in practice, the FS has never been accumulated in a separate escrow account, so it is very difficult to know, at any point in time, the "balance" of the FS.

Revenue loses. The current non-automatic system is prone to causing revenue losses due to the pressure not to adjust prices at different points in time depending of the evolution of international fuel prices.

\footnotetext{
${ }^{4}$ Take for example a situation in which import prices have been increasing continuously and the government has decided, in each period, to keep retail prices unchanged (depleting gradually resources from the FS). When facing a new rise in import prices, and bearing in mind the limited balance in the FS, the government would need to decide whether to translate the entire increase to retail prices (full pass-through, i.e. stop withdrawing resources from the FS); translate some of the increase to retail price (incomplete pass-through, withdrawing some resources from the FS); or keep retail prices unchanged (zero pass-though, heavily withdrawing resources from the FS). Sooner or later the government will be faced with a situation in which import prices keep increasing and the FS is exhausted. At that time it might be forced to pass a large increase to retail prices to catch up with the price that would have prevailed in the absence of a FS mechanism.
} 


\section{REVISITING BURUNDI'S FUEL PRICE SMOOTHING MECHANISM}

\section{A. Motivation for an Automatic Fuel Pricing Mechanisms}

Why adopt an automatic fuel price mechanism? An automatic mechanism is intended to guarantee full pass-through of international prices to domestic prices, which in turn ensures that consumers face the true opportunity cost of fuel. It also avoids subsidies which might lead to: inefficiently high levels of consumption; distortive taxation to finance them; high volatility of tax revenue (affecting, in turn, priority public spending).

Why add a price smoothing component? The adoption of an automatic fuel price mechanism that encompasses an automatic price smoothing mechanism can ensure full pass-through over the medium term while also avoiding sharp price increases. Tax revenue volatility would not necessarily be higher than under the irregular adjustments that prevail when the government follows a "waitand-see" approach (see section II.B).

A range of automatic price smoothing mechanisms is available. Within the class of automatic mechanisms, a recent FAD technical note draws attention on 2 groups of them: price band and moving average mechanisms:

- Price band mechanisms (PB): This approach sets a maximum limit on the magnitude of retail price changes, in both directions. Caps can be set as a proportion of the current retail price or in absolute amounts (although absolute caps can become less effective as international prices increase). Under price caps, at the start of each period (e.g., month) the retail price according to the full pass-through mechanism is determined based on last month's average import cost. If the required retail price increase is above the maximum allowed increase (i.e., the cap), then the maximum allowed increase is implemented and net taxes adjusted accordingly. If the implied price increase is below this threshold, then the full adjustment is allowed. The same logic applies for downward adjustments.

- Moving average mechanisms (MA): This approach bases retail price adjustments on changes in the average of past import costs: At the start of the period (e.g. month) the retail price under the formula is calculated using an average of past import costs (say, the average of import costs for the last three months). Retail prices are then allowed to fully adjust to the smoothed formula import price. The difference with the retail prices that would have prevailed under full passthough is absorbed by net taxes. 


\section{B. Adopting an Automatic Price Smoothing Mechanism in Burundi}

Adopting an automatic mechanism would imply:

i. fluctuations in tax revenue in the short-term - though in an automatic and transparent way-but no fiscal loss over the medium-term;

ii. a delayed pass-through but in an automatic way, avoiding the costs and political pressure faced when the adjustment is decided in an ad hoc way.

In practical terms, substituting the FS with an automatic price smoothing mechanism would only entail adjusting "net taxes" (grouping custom duties, excise tax and VAT together with the actual contribution to the FS) every month in an automatic way as prescribed by the rules of the smoothing mechanism.

How would have an automatic price smoothing mechanism worked for Burundi? Based on simulations under alternative MA and PB mechanisms (plotted in Figure 1) and indicators of their variability, performance under PBs and MAs are compared to the current system using the FS (denoted HIST in Tables and Figures) and to the one that would have prevailed if a full pass-through mechanism had been in place (labeled FPT). ${ }^{5}$ Table 1 reports the volatility results for retail prices and for tax revenue. ${ }^{6}$ Table 2 presents results for tax levels, and Table 3 presents an overall ranking, taking into account the performance in terms of minimizing tax and price volatility while safeguarding tax revenue.

The current system (HIST) is ranked as the worst mechanism in terms of the standard deviation of prices and of the largest single monthly price increase indicators (Table 1 ). The highest rankings belong to the MAs with wide windows ( 6 and 4 months) and the PB with a narrow band (3 percent). ${ }^{7}$

\footnotetext{
${ }^{5}$ The price and tax series shown in Figure 2, and used for the indicators in Tables 1-4, correspond to a "basket" fuel taking into account the average consumption of gasoline, diesel and kerosene. The data available for this exercise is from January 2009 to August 2011. As Figure 1 shows, this period is characterized by an upward trend in international prices, which conditions the results of this analysis. An extended dataset with complete cycles in international prices would have been preferred.

${ }^{6}$ PB3, PB5 and PB10 denote 3 percent, 5 percent and 10 percent price band mechanisms. MA2, MA4 and MA6 refer to moving average mechanisms using import prices from the last 2, 4 and 6 months respectively.

${ }^{7}$ As a reference, note that a PB3 for Burundi would currently imply a maximum price increase or decrease of about $60 \mathrm{Fbu}$.
} 
Table 1. Volatility Ranking

\begin{tabular}{|c|c|c|c|c|c|c|c|c|}
\hline Price volatility & FPT & HIST & MA2 & MA4 & MA6 & PB3 & PB5 & PB10 \\
\hline Std. deviation of monthly prices & 7 & 8 & 4 & 2 & 1 & 3 & 5 & 6 \\
\hline Avg monthly price change (sq) & 8 & 2 & 4 & 3 & 1 & 5 & 6 & 6 \\
\hline Largest single monthly price increase & 5 & 8 & 4 & 2 & 1 & 3 & 5 & 5 \\
\hline Avg of 2 largest monthly price inceases & 6 & 5 & 3 & 2 & 1 & 4 & 6 & 6 \\
\hline Avg of 3 largest monthly price increases & 6 & 5 & 4 & 2 & 1 & 3 & 6 & 6 \\
\hline Sum of price volatility rankings & 32 & 28 & 19 & 11 & 5 & 18 & 28 & 29 \\
\hline Rank order & 8 & 5 & 4 & 2 & 1 & 3 & 5 & 7 \\
\hline Tax volatility & FPT & HIST & MA2 & MA4 & MA6 & PB3 & PB5 & PB10 \\
\hline Std. deviation of taxes & 1 & 7 & 4 & 6 & 8 & 5 & 3 & 2 \\
\hline Avg monthly tax change (sq) & 1 & 3 & 5 & 7 & 8 & 6 & 4 & 2 \\
\hline Largest single monthly tax decrease & 1 & 4 & 8 & 5 & 6 & 3 & 7 & 2 \\
\hline Avg of 2 largest monthly tax decreases & 1 & 4 & 3 & 8 & 6 & 7 & 5 & 1 \\
\hline Avg of 3 largest monthly tax decreases & 1 & 5 & 4 & 8 & 7 & 6 & 1 & 1 \\
\hline Sum of tax volatility rankings & 5 & 23 & 24 & 34 & 35 & 27 & 20 & 8 \\
\hline Rank order & 1 & 4 & 5 & 7 & 8 & 6 & 3 & 2 \\
\hline
\end{tabular}

Source: Staff estimates.

Note: Rank of (1) represents highest ranking in terms of minimizing volatility of taxes and prices.

Regarding tax levels, Table 2 reveals that, in the sample considered, any of the PB or MA mechanisms would have performed better than the current FS scheme (labeled HIST in the Table). Indeed, fiscal loses under the current system seem to have been sizeable (see Appendix A). Furthermore, for the sample considered, a price band mechanism would have performed best in terms of safeguarding tax revenue in Burundi, as they imply only a temporary gap between the price under the formula and the one that would prevail under full pass-though.

\begin{tabular}{lccccccccc}
\multicolumn{1}{c}{ Table 2. Net Tax Level } & & & PA3 & PB5 & PB10 \\
\hline & FPT & HIST & MA2 & MA4 & MA6 & PB3 \\
\cline { 2 - 10 } Monthly average of net taxes (ranking) & 4 & 8 & 5 & 6 & 7 & 1 & 2 & 3 \\
\hline Source: Staff estimates. & & & & &
\end{tabular}

Note: Rank of (1) represents highest ranking in terms of maximizing tax levels.

The overall ranking shown in Table 3 places the current mechanism as the worst performer. The price band mechanisms, instead, would have been the best performers during the sample period analyzed.

Table 3. Overall Ranking

\begin{tabular}{|c|c|c|c|c|c|c|c|c|}
\hline & FPT & HIST & MA2 & MA4 & MA6 & PB3 & PB5 & PB10 \\
\hline Price volatility & 8 & 5 & 4 & 2 & 1 & 3 & 5 & 7 \\
\hline Tax volatility & 1 & 4 & 5 & 7 & 8 & 6 & 3 & 2 \\
\hline Tax level & 4 & 8 & 5 & 6 & 7 & 1 & 2 & 3 \\
\hline Overall ranking & 4 & 8 & 5 & 6 & 7 & 1 & 1 & 3 \\
\hline
\end{tabular}




\section{Figure 1 - Fuel Prices under Alternative Smoothing Mechanisms}

Historical (Stabilization Fund)

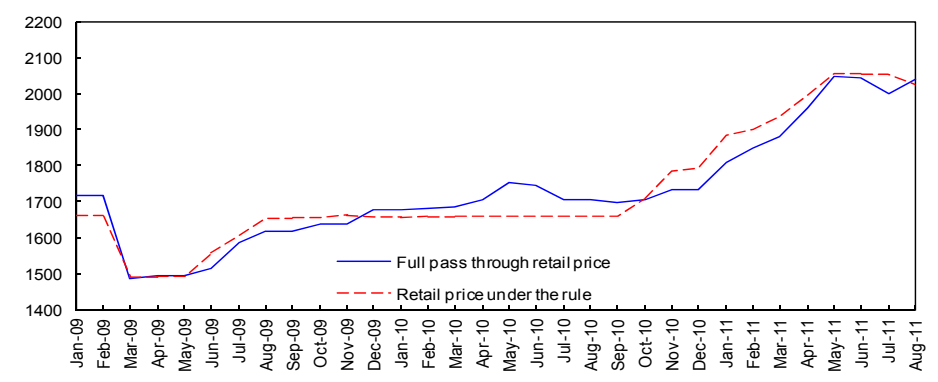

Price Band, 5\%

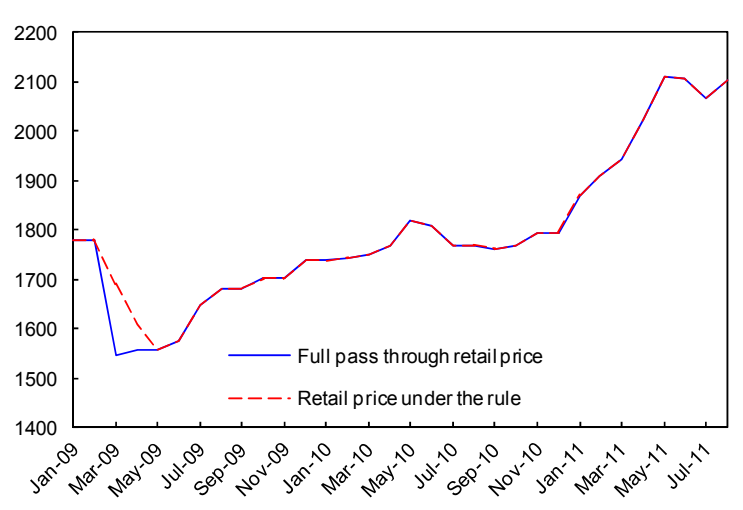

Moving Average, 4 Months

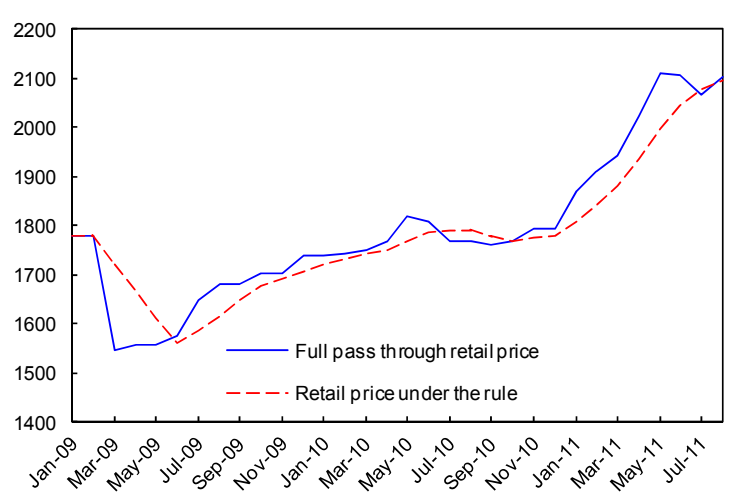




\section{APPENDIX A}

We computed actual and theoretical net tax series since January 2010 (see Figure below). Net taxes include excise taxes, VAT and contributions to the funds (both FS and strategic fund). The "theoretical" level of net taxes is the one that would have prevailed if the excise taxes were at their original levels (246.41, 234.85 and 54.7 BIF per liter of gasoline, diesel and kerosene respectively) and the FS and the strategic fund did not exist (i.e. their contributions were set to zero). The actual net tax series is the actual revenue from excise taxes, contributions to the funds and VAT. The fiscal loss plotted is the product of the unitary (i.e. per liter) fiscal loss, that is, the difference between actual and theoretical net taxes, times the actual volumes imported. We use the price formula for each month and actual imported quantities up to July 2011 (for August and September we assumed imports are equal to the average in May-July 2011). ${ }^{1}$

The Figure below reveals that the gap between the actual and theoretical net tax has widened significantly in the recent conjuncture for gasoline and diesel, but not for kerosene. While the actual net tax for kerosene decreased in May, when the subsidy was implemented, this was reversed in the following months: in August 2011 the only fuel that was paying the theoretical level of excise tax was kerosene. Instead, the net subsidy per liter of gasoline and diesel has kept increasing up to September.

We also estimate the cost of the current fuel subsidy policy Burundi introduced in May 2011 (see Table below). We consider that a subsidy has been in place only since May 2011 onwards since it is argued that, before then, the cumulated contributions to the funds (both the FS and the strategic fund) were above the revenues losses (due to lower excise taxes). However, the fund(s) are assumed to have been depleted by May 2011. We use the price formula for each month and actual imported quantities in May-July.

Reference Import Cost (basket, BIF/liter)
Retail Price (basket, BIF/liter)
Monthly Revenue Loss (all fuels, billion BIF)
Gasoline
Diesel
Kerosene
Cumulative Revenue Loss (all fuels, billion BIF)

$\begin{array}{ccccc}\text { May-11 } & \text { Jun-11 } & \text { Jul-11 } & \text { Aug-11 } & \text { Sep-11 } \\ 1,344 & 1,347 & 1,306 & 1,341 & 1,349 \\ 2,056 & 2,056 & 2,056 & 2,026 & 2,026 \\ \mathbf{0 . 6 2} & \mathbf{0 . 6 0} & \mathbf{0 . 4 0} & \mathbf{0 . 9 9} & \mathbf{1 . 1 9} \\ 0.06 & 0.23 & 0.02 & 0.27 & 0.41 \\ 0.55 & 0.37 & 0.38 & 0.72 & 0.78 \\ 0.01 & 0.00 & (0.00) & 0.00 & 0.00 \\ \mathbf{0 . 6 2} & \mathbf{1 . 2 2} & \mathbf{1 . 6 2} & \mathbf{2 . 6 1} & \mathbf{3 . 8 1}\end{array}$

\footnotetext{
${ }^{1}$ Along this section we are using the price formula for "Depot Bujumbura" for all quantities imported. Although the formula used for Gitega entails slightly different level of contribution to the funds, this entry point represents, on average, less than $10 \%$ of the imported volume.
} 
The cumulated revenue losses up to September are estimated at almost 4 billion BIF. The bulk of the subsidy cost, almost $3 / 4$, has benefited diesel consumption, while less than 1 percent of the subsidy went to kerosene consumption in this period. The revenue loss for 2011 is 10.6 billion BIF (0.36 percent of GDP). To summarize, total costs of subsidies per year, are about $1 / 3$ of a percent of GDP.

Fiscal Loss, Annualized, in \% of GDP

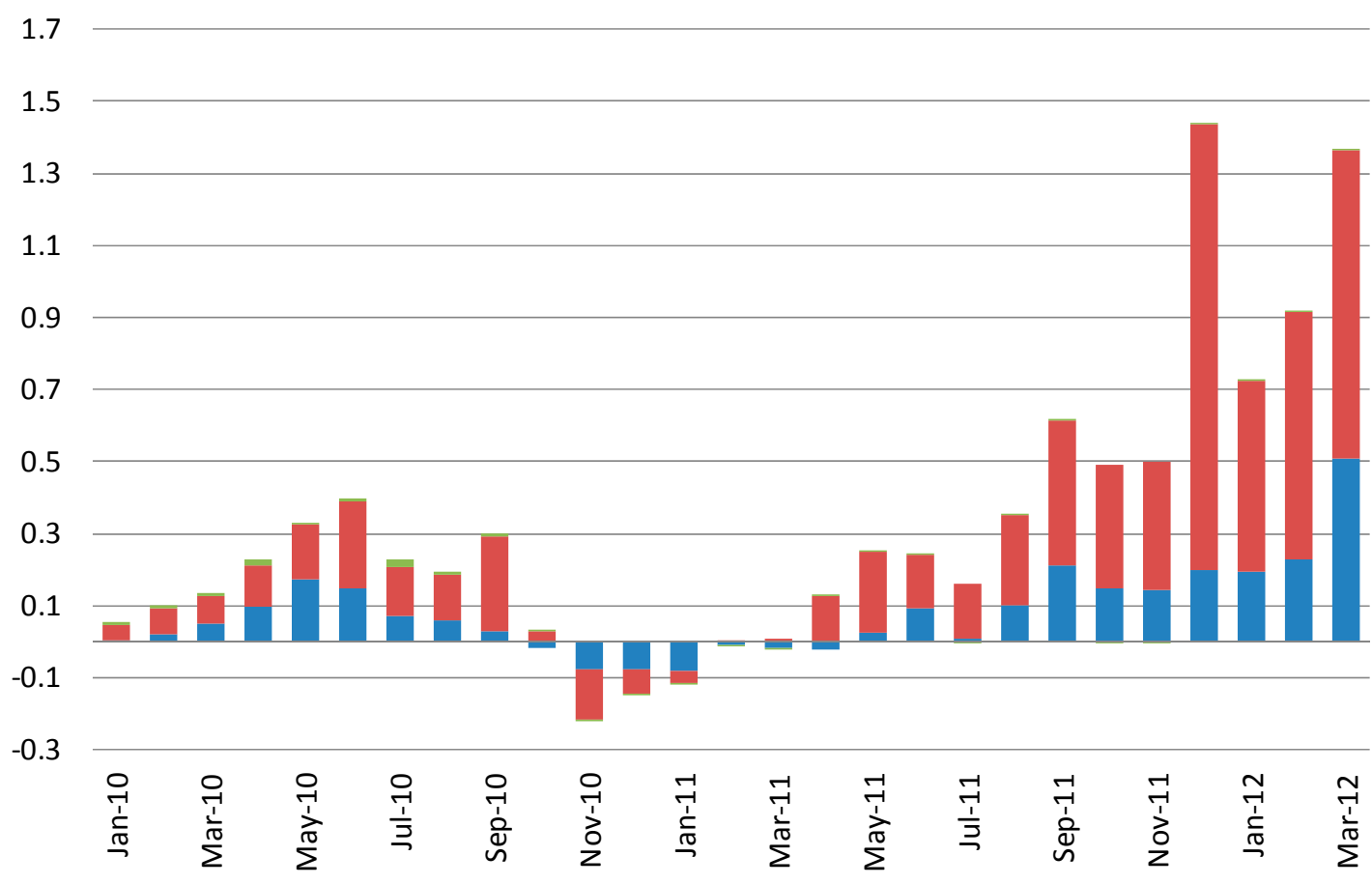

Fiscal Loss, Gasoline (\% GDP) $\square$ Fiscal Loss, Diesel (\% GDP) $\square$ Fiscal Loss, Kerosene (\% GDP) 


\section{ANNEX III. BURUNDI: EXCHANGE RATE ASSESSMENT ${ }^{1}$}

\section{A. Recent Developments}

\section{After a long period of stability, the Burundian Franc demonstrated greater flexibility in 2011.}

It depreciated by about 12 percent against the US dollar in the course of 2011, leading the IMF to reclassify the country's exchange rate regime from 'pegged' to 'other managed'. Faced with higher imported inflation, the effective exchange rate (NEER) depreciated by about 4 percent (Figure 1$)^{2}$. The real effective exchange rate (REER) appreciated by a cumulative 15.7 percent during 2008-10, reflecting higher inflation relative to trading partners, before depreciating slightly by 0.3 percent in 2011. Reserves in months of imports also trended down at end2011, reflecting the persistence of the food and fuel shocks, lower aid inflows

Figure 1. Real Effective Exchange Rate Developments

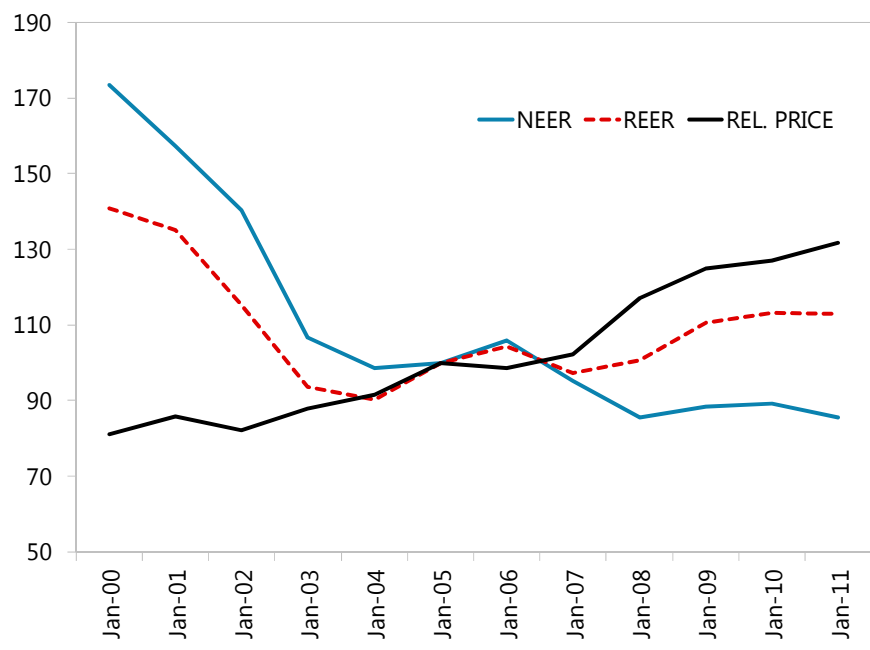

Source: Burundi authorities and IMF staff estimates. and the higher costs of fuel-related imports.

\section{Burundi, like other members of the East African Community (EAC) faced a common shock but} reacted much slower in allowing the currency to depreciate. In the first three quarters of 2011 , food inflation rose to peaks of 26 percent in Kenya, boosted by local food shortages in the wake of the drought. Burundi, Tanzania, and Uganda saw food inflation rise to 20 percent, 22.5 percent, and 46 percent, respectively. In addition to food, higher transportation costs associated with rising global oil prices had a particularly important impact on consumer prices in landlocked Burundi and Uganda, while power tariffs were hiked sharply in Tanzania. The increase in the region's nominal exchange rates against the dollar also fueled the inflationary process. Against the US dollar, local currencies depreciated in Kenya, Uganda, and Tanzania by 11 percent, 10 percent, and 16 percent, respectively, in the first three quarters of the year (Figure 2), before starting to appreciate following

\footnotetext{
${ }^{1}$ Prepared by François Boutin-Dufresne.

${ }^{2}$ The EU accounts for about 47 percent of Burundi's exports and 22 percent of its imports.
} 
the tightening of the monetary stance. The recent 12 percent depreciation of the Burundian franc is thus in line with regional trends.

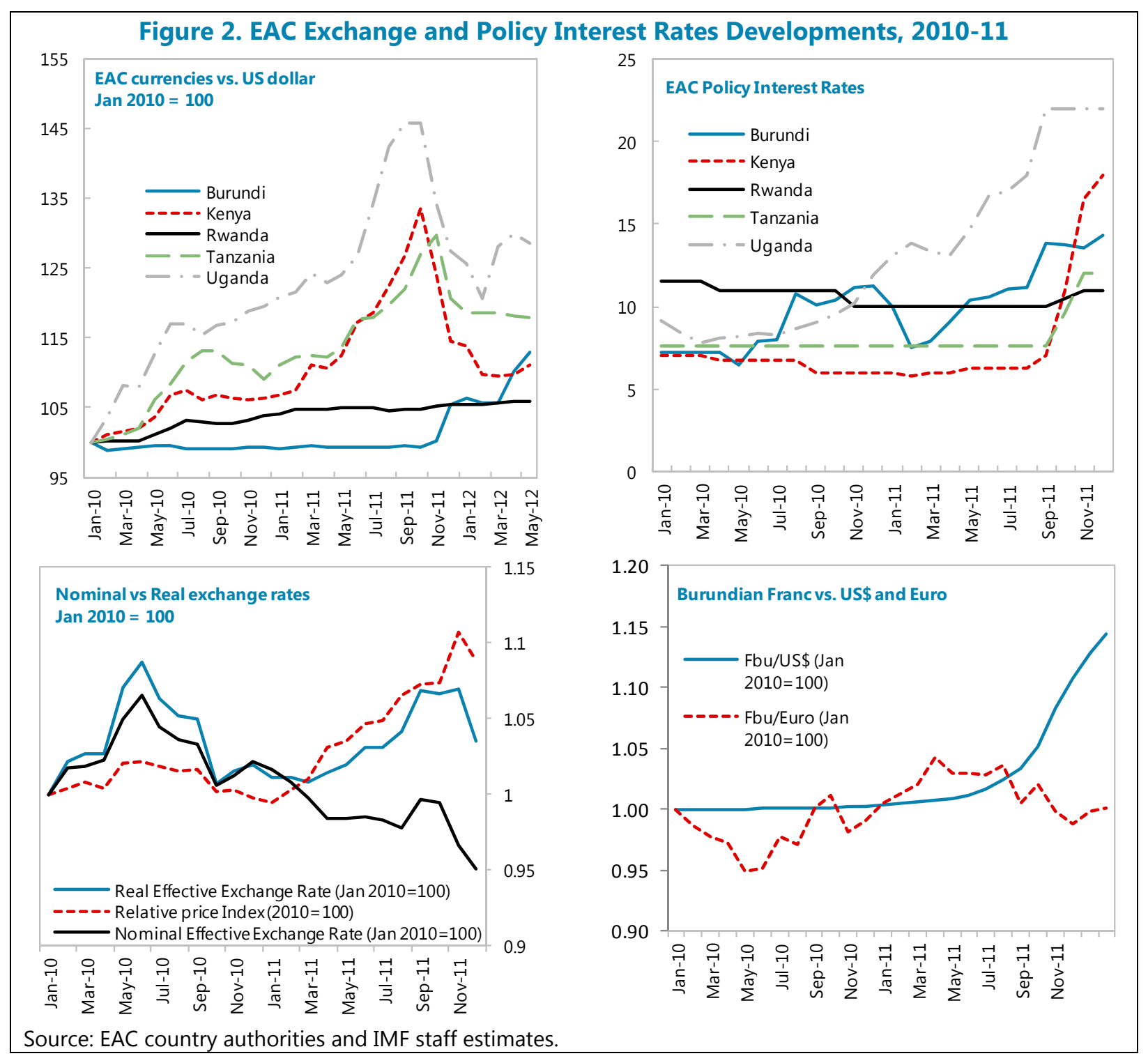

\section{Beginning in September 2011, the authorities stepped in more firmly to curb potential}

second-round effects of high inflation. Lower-than-expected aid inflows also brought pressure to bear on the currency in concert with the persistence of the food and fuel shock. In response to these developments, the authorities raised policy rates by an additional 331 basis points (bps) since July 2011 (Figure 2), compared to Kenya (1175 points), Tanzania (442 points), Uganda (1000 points), and Rwanda (100 points). 


\section{Burundi's current account deficit improved somewhat since the end of the civil war, but} remains in deep negative territory. Burundi's current account excluding grants, stabilized following the 2005 peace agreement, but the country still remains highly dependent on foreign aid flows. The current account deficit, excluding grants, averaged about 20 percent of GDP since 2000. Despite a significant improvement in the terms of trade since 2000, the trade deficit averaged 24 percent of GDP owing in part to Burundi's narrow export base and rising imports as aid was spent and absorbed. The capital and financial account benefitted from one time debt relief in 2009 worth about US\$1 billion.

\section{Burundi's trade openness increased as aid inflows were spent and absorbed. Openness}

measures by exports and imports to GDP rose from 24 percent in 2000 to 51 percent at end-2011. Coffee prices and export volumes rose threefold over the period. Imports also rose sharply in value, mainly through the absorption of official aid flows. During the post conflict period, official transfers, grew four fold since 2000, before declining during the latter part of the decade. Remittances also increased substantially after the conflict, and represent a third of current transfers. 
Figure 3. Developments in the Balance of Payments, 2000-11

(percent of GDP)

The current account improved since the end of the conflict, but remains in deep negative territory...

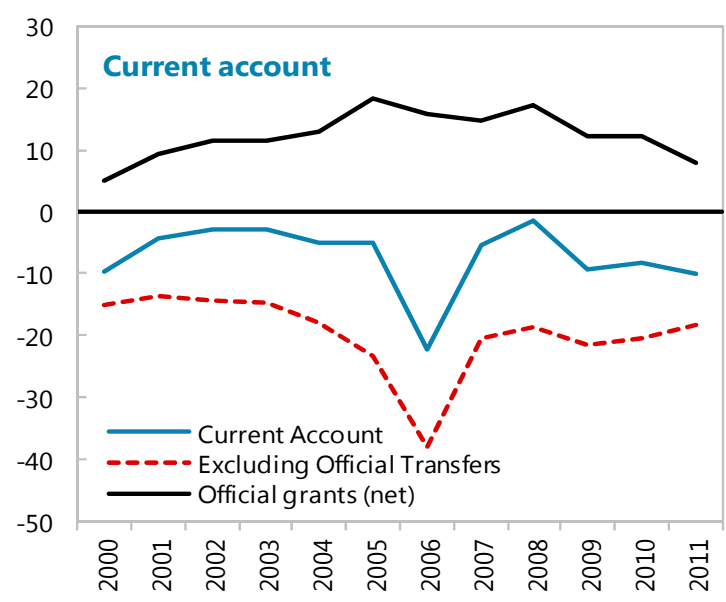

Project grants grew sharply as donors supported the reconstruction process...

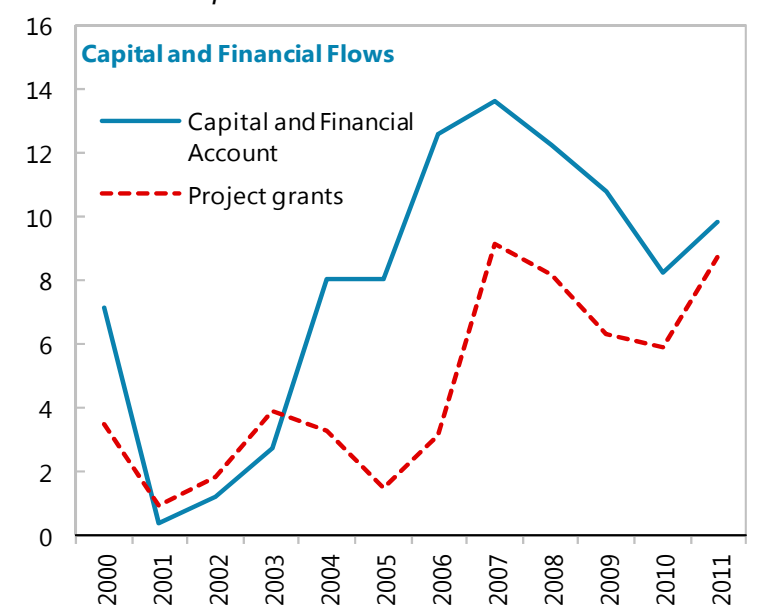

The overall balance deteriorated because of the food and fuel shocks and lower aid flows...

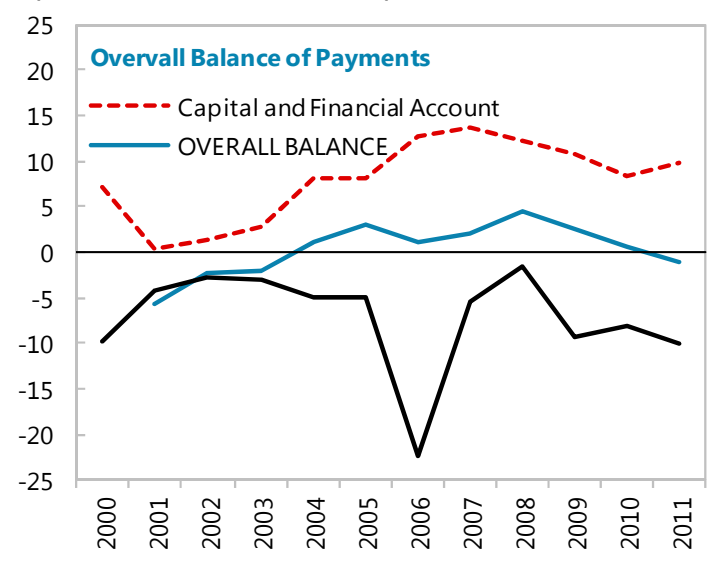

Source: Burundi authorities and IMF staff estimates. ...as rising coffee prices did not offset the rising price of oil.

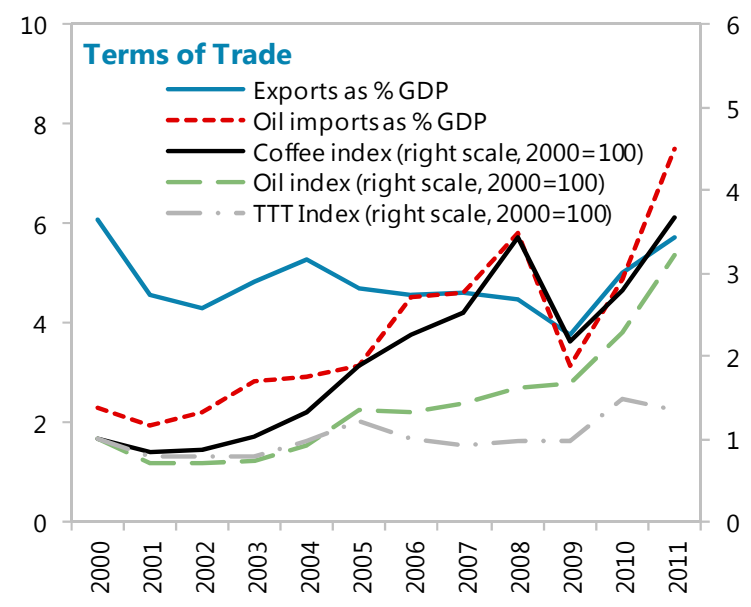

...but current transfers declined substantially since 2008.

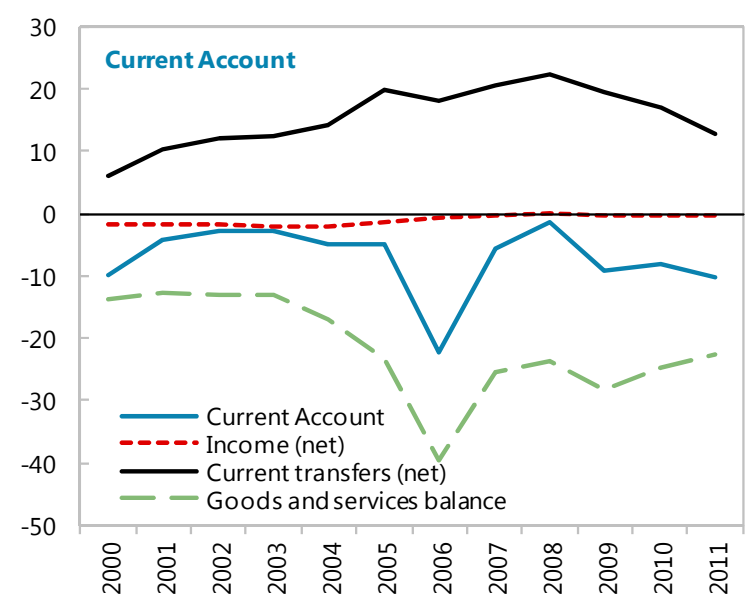

...which is the most important component of the capital account.

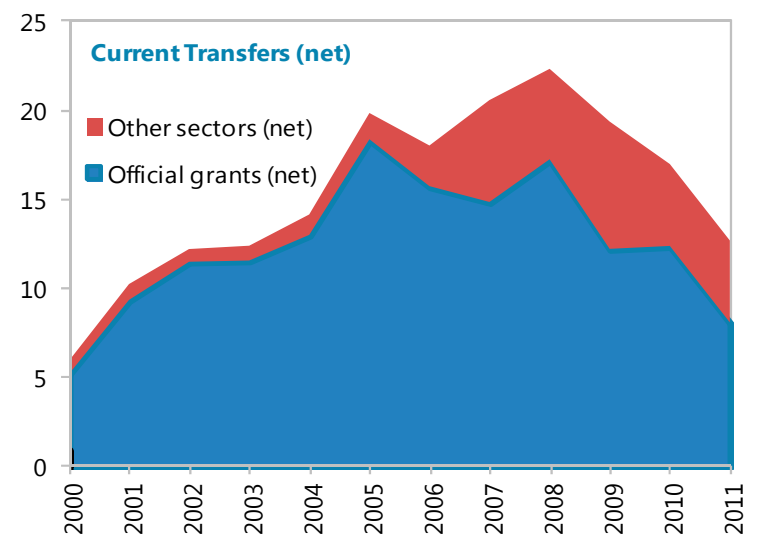




\section{B. Exchange Rate Assessment}

Data shortcomings in Burundi limit the effectiveness of typical CGER methodologies. The external competitiveness assessment of some low income countries may require the use of non traditional methods to assess the level of the REER. Given data shortcomings in Burundi, two approaches were used; (i) econometric, using real coffee prices to estimate the equilibrium exchange rate (EREER); and (ii) an assessment of Burundi's relative productivity to that of its trading partners, based on a trade weighted relative productivity index.

\section{Following Cashin et al. (2004) commodity currency methodology, the REER was regressed on}

real coffee prices ${ }^{3}$. This was predicated on coffee accounting for 85 percent of exports and because of the lack of a long time series on other macroeconomic fundamentals. One cointegrating vector was found between the REER and real coffee prices. The error correction term was significant, suggesting that although these two series are subject to transitory deviations, they tend to revert to an equilibrium relationship. The results suggest that Burundian franc is undervalued by about 8 percent in 2011 (Figure 4).

Figure 4. Real Equilibrium Exchange Rate, 2000-11

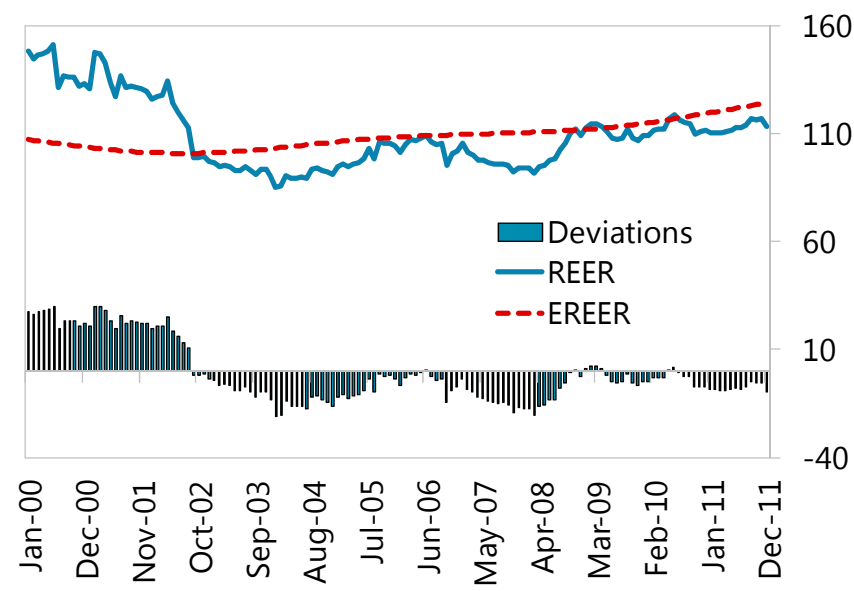

Source: Burundi authorities and IMF staff estimates.

These results should be interpreted with caution. These imply that the Burundi Franc should appreciate to close the gap between the REER and its equilibrium level (EREER) which would likely worsen Burundi's competitiveness. Notwithstanding, the projected deterioration in the terms of trade by 18 percent in 2012 would help to close this gap by lowering the level of the EREER. The large role played by aid inflows in financing both the current account and fiscal deficits contributed to the strengthening of the currency over time.

\footnotetext{
${ }^{3}$ Cashin et al (2004) "Keynes, Cocoa and Copper: In Search of Commodity Currencies" IMF WP 02/223. Real coffee prices were computed using US CPI as the deflator.
} 
The second approach analyzes the trends in

\section{Burundi's productivity relative to its trading}

partners. ${ }^{4}$ Relative productivity has declined

sharply during the civil war, before stabilizing at about half of its pre-war level (Figure 5).

Productivity has declined in the face of large aid inflows which have been largely absorbed during reconstruction in the post conflict period.

\section{Burundi suffers from several structural issues}

that affect its competiveness. Despite making

progress in improving its business environment in
Figure 5. Relative Productivity and REER, 2000-11

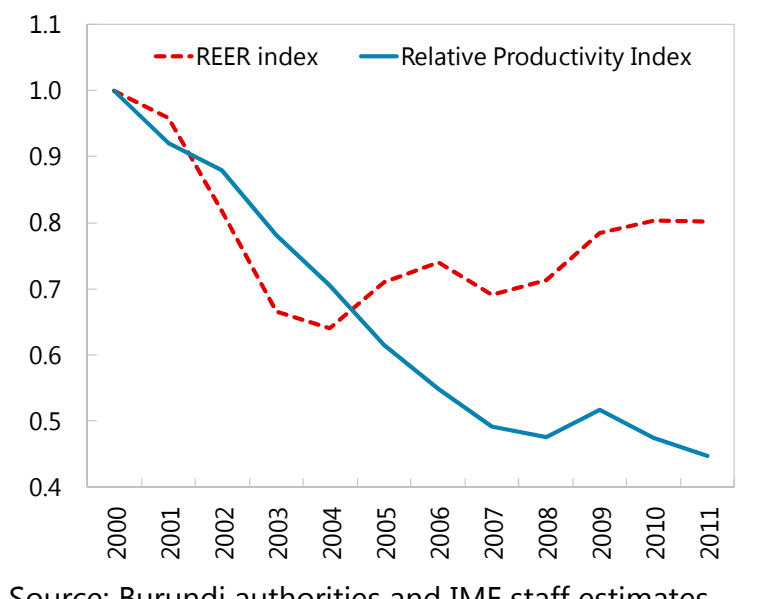

Source: Burundi authorities and IMF staff estimates.

2011, the country still ranked $169^{\text {th }}$ (out of 183 countries) on the World Bank's 2012 Doing Business

Index, and $140^{\text {th }}$ (out of 142 countries, Figure 6) on the World Economic Forum's Global

Competiveness Index. Both reports cited corruption and access to finance as two major

competitiveness hurdles for local businesses, which continue to rank Burundi as one of the least

competitive and business friendly countries in the world. Transparency International ranked the

country 172 out of 183 on its Corruption Perception Index (2011). Being a landlocked country,

Burundi's suffers from poor

transport and logistical links to

and from regional ports in

Tanzania and Kenya ${ }^{5}$. As a

result, shipping costs to import

a 20 foot container in Burundi

are the highest in the EAC

(Figure 6). Other factors

impacting competitiveness,

include poor electricity supply

which was cited by 72 percent of businesses as the main impediment to growth (IMF Country Report

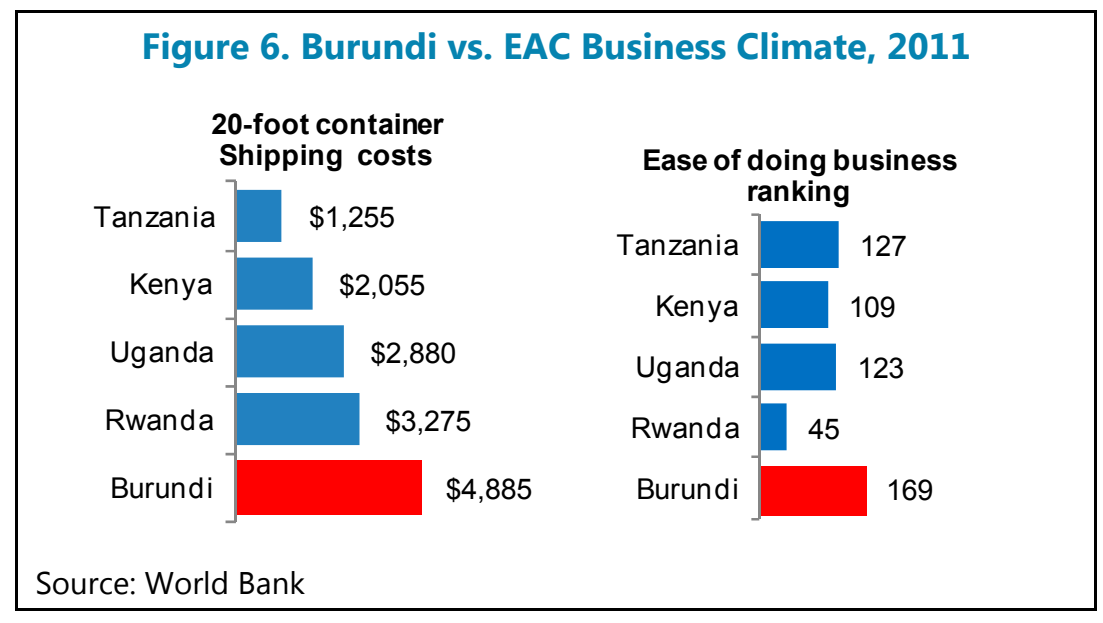
$12 / 28)$.

\footnotetext{
${ }^{4}$ Relative productivity is defined as the ratio of Burundi's real per capita GDP to that of its main trading partners.

${ }^{5}$ The World Bank estimates that the cost of importing or exporting a 20-foot container to Burundi was 4855 US dollars in 2011, one of the highest in the world. The cost measures the fees levied on a 20-foot container in U.S. dollars.
} 


\section{Conclusion}

\section{Assessing the potential misalignment of a currency is challenging in a low-income fragile}

state such as Burundi. While the commodity currency model suggests a slight undervaluation, this result should be taken with caution. Other indicators suggest that Burundi needs to improve its competitiveness while the development of a productive private sector remains a challenge given the many obstacles of the current business environment. Limited access to electricity and frequent outages further stymie productivity. 


\title{
APPENDIX I: LETTER OF INTENT
}

Bujumbura, July 9, 2012

\author{
Madame Christine Lagarde \\ Managing Director \\ International Monetary Fund \\ Washington, D.C. 20431
}

Dear Madame Lagarde:

1. On January 27, 2012, the Executive Board of the International Monetary Fund (IMF) approved a new three-year arrangement under the Extended Credit Facility (ECF) in favor of the Republic of Burundi. This arrangement is intended to support our medium-term program and to strengthen macroeconomic stability, expedite growth, and reduce poverty. Under this arrangement, the Burundi government recently discussed the first program review with an IMF mission. This review focused on program implementation from December 1, 2011 to March 31, 2012, as well as on the outlook and the economic and financial measures to be implemented in 2012.

2. Since the beginning of the year, Burundi has been confronted with an unfavorable international economic environment, characterized by declining budgetary assistance and rising food and oil prices. Our forecasts suggest that these trends will have adverse effects on economic growth, balance of payments, and budget execution in 2012. To mitigate the impact of this crisis on the cost of living for vulnerable population groups, the government has implemented tax relief measures on food and petroleum products whose fiscal effects will be partly offset by higher taxes on alcoholic beverages, telephone communications, second-hand cars, along with a substantial reduction in domestically funded expenditures. These measures were incorporated into a supplementary budget that was submitted to parliament.

3. These shocks notwithstanding, program implementation at end-March 2012 was satisfactory overall. All performance criteria and indicative targets were observed. In addition, the government intends to press ahead with the program in pursuit of the objectives of fiscal and debt sustainability, 
and to strengthen the economic growth outlook for Burundi in the medium term. In particular, the government intends to tighten short-term fiscal and monetary policies in an effort to curb inflationary pressures, while safeguarding pro-poor expenditure. The government also stands ready to adopt any additional measures that may be required for this purpose. The government will consult with the IMF in advance of the adoption of such measures and/or of revisions to the policies contained in the MEFP, in accordance with the IMF's policies on such consultations.

4. With regard to the structural reforms, in spite of being delayed in the implementation of reforms, the Government is determined to adopt the decree of budget governance and to recruit an international auditor to reinforce the safeguards at the central bank and the ministry of finance by mid-July 2012. Because of this delay, the audit report will be made available at the end of September 2012.

5. The PRSP-II aims to achieve strong and sustained growth based on transformation of the economy financed through the mobilization of domestic resources and grants. In the event that grants are lower than expected, the government will undertake appropriate measures to realign the macroeconomic framework with available resources.

6. The government will provide the IMF with such information as it may request to ensure implementation of the program. That information as well as arrangements for monitoring implementation of the program and the performance criteria, quantitative targets, and structural benchmarks are detailed in the Technical Memorandum of Understanding, which is also attached to this letter.

7. In light of the appreciable progress in implementing the program supported by the ECF arrangement, the government is requesting the modification of PCs for end-September 2012 and completion of the first review as well as the second disbursement of SDR 4 million under the ECF.

8. The Burundi authorities wish to make this letter available to the public, along with the attached MEFP and Technical Memorandum of Understanding (TMU), as well as the IMF staff report on the first review. We therefore authorize their publication and posting on the IMF website, subject 
to Executive Board approval. These documents will also be posted on the official websites of the Burundian government.

Sincerely yours,

/s/

Tabu Abdalla MANIRAKIZA

Minister of Finance and Economic Planning /s/

\section{Gaspard SINDAYIGAYA}

Governor, Bank of the Republic of Burundi

/s/

Gervais RUFYIKIRI

Second Vice President, Republic of Burundi

Attachments:

Memorandum on Economic and Financial Policies (MEFP)

Technical Memorandum of Understanding (TMU) 


\section{ATTACHMENT I. MEMORANDUM ON ECONOMIC AND FINANCIAL POLICIES, 2011-14}

\section{INTRODUCTION}

1 Burundi's economic and financial program, supported by the International Monetary Fund (IMF) under the Extended Credit Facility (ECF), aims to consolidate economic and political gains, promote inclusive economic growth, contain inflation, and strengthen policies designed to combat endemic poverty in rural and urban areas. This Memorandum supplements the December 2011 version. It reports on quantitative implementation at end-March 2012, structural benchmarks through end-June 2012 and defines the economic policies and reforms the government intends to implement in 2012 to achieve the objectives of its economic and financial program. The program measures and objectives are consistent with the Poverty Reduction and Growth Strategy Paper (PRSP-II).

2 Despite remarkable progress, Burundi remains a fragile state. It still needs technical and financial support from the U.N. and other bilateral agencies to complete the transformation of its economy and strengthen its political institutions. In the interest of creating a calm social and political environment, dialogue is the government's preferred method of managing conflicts. It was in this context that the government, acceding to civil society's demand to eliminate taxes on food, forestalled a strike protesting the high cost of living.

\section{ECONOMIC DEVELOPMENTS AND IMPLEMENTATION OF THE ECONOMIC AND}

\section{Financial Program in 2011/Early 2012}

\section{A. Recent Economic Developments and Outlook for End-2011and March 2012}

3. Economic activity improved slightly to $\mathbf{4 . 2}$ percent in 2011 , falling a little short of the initial projections. This growth reflected the expansion of services and major foreign investments in the secondary sector. Agricultural output, however, was much weaker than expected owing to the cyclical decline in coffee production and a decrease in food production caused by bad weather. Inflationary pressures mounted, with the rate of inflation rising to 25 percent at end-March 2012, compared to 15 percent at end-December 2011. Prices rose sharply in response to the relatively high prices of foodstuffs on the international market and a drop in agricultural output of 
approximately 30 percent in the first quarter of 2012. The government's decision to raise electricity and water rates also stoked inflation.

4. The current account (including transfers) widened further to 12.3 percent of GDP in 2011. Terms of trade deterioration and delays in budgetary assistance disbursements largely explain this unfavorable development. Gross official reserves contracted sharply, to a level equivalent to 4 months of imports in 2012.

5. The monetary survey at end-December 2011 reveals a sharp decrease in net foreign assets of roughly 12.3 percent of the beginning period money supply, a substantial increase in credit to the economy, and money supply growth of 6.1 percent. The decrease in net foreign assets is attributable to delays in the disbursement of budgetary assistance and the decision of the monetary authorities to stabilize the Burundian franc by selling larger amounts of foreign exchange on the exchange market. Rising oil and food prices also contributed to the decline in net foreign assets. Overall in 2012 , credit to the economy will remain moderate at 23 percent owing to the tightening of monetary policy to curb inflationary pressures. Furthermore, with a view to mitigating the effects of external shocks on domestic production, the country's foreign exchange policy has become more flexible. The daily reference exchange rate is now determined using the weighted average of the rates applied by commercial banks to the previous day's purchases and sales of foreign exchange. Foreign exchange auctions are held twice a week, with foreign exchange allocated at the rates proposed by banks.

6. On the fiscal front, the mobilization of domestic funds improved, as projected, and expenditure was kept within the program envelope. The overall deficit, however, was larger than anticipated owing to the delay in budgetary assistance disbursements in 2011. At end-December 2011, the overall budget deficit (cash basis, non-HIPC grants included) was approximately 4 percent of GDP, well above the target of 2.5 percent. Domestic revenue amounted to 15.4 percent of GDP thanks to the revenue mobilization efforts of the Burundi Revenue Authority (BRA). Total spending fell short of projections by about 0.4 percentage points of GDP as a result of a decrease of FBu 5 billion in transfer expenditures. However, pro-poor spending of roughly FBu 372.6 billion was broadly in line with the budgetary objectives and helped mitigate the negative effects of energy and food crisis on vulnerable populations.

7. At 8.7 percent of GDP in 2011, the wage bill exceeded projections by nearly FBu 1.6 billion, or 0.05 percent of GDP, owing to the hiring of more personnel in the health and education sectors than planned. The reason for this overage is that careers and payroll are managed by the Ministry of the Civil Service, although they could be brought under control if payroll management responsibilities were transferred to the Ministry of Finance and Economic Development Planning. 
8. At end-May 2012, revenues were lower than expected owing to the granting of exemptions and lower collections on excise taxes (by about 0.5 percent of GDP). To counter the high cost of living, the government provided tax relief by eliminating customs duties, VAT, and administrative charges on 13 food products. This tax exemption applies to both local and imported products. For 2012 , the amount of forgone revenue associated with this decision is estimated at FBu 10 billion, or 0.3 percent of GDP. Similarly, because of rising international oil prices, the government waived excise taxes on oil products to offset soaring retail prices and their effect on inflation. At endDecember 2012, the loss of revenue caused by this subsidy is projected at FBu 23 billion. Nevertheless, corrective measures are envisaged to address the slippages.

9. Program implementation is satisfactory overall. The indicative targets for end-December 2011 were met but for that on pro-poor spending that was missed by a small margin. At end-March 2012, the quantitative performance criteria were met, as well as all the indicative criteria.

\section{Implementation Of Structural Measures}

10. Regarding public financial management (PFM), the government recently concluded the implementation of an initial public financial management strategy (PFMS-1), as well as a 2009-11 action plan. Early assessments indicate that substantial progress has been made on several aspects of PFM, particularly modernization of the legal and institutional framework of PFM and budget programming and preparation. As a result, significant gains were achieved in the areas of budget credibility, supervision, cash management, and government accounting. Building on this progress, a new strategy for 2012-14, aimed at consolidating gains, will be developed in collaboration with our technical and financial partners. With regard to revenue collection, the government is continuing its efforts to have a lasting impact on revenue. In addition to its campaign to raise taxpayer awareness, the government has simplified customs clearance procedures and created a one stop border post with Tanzania to facilitate the movement of goods across that border, which is the point of entry for a large portion of our foreign trade.

11. On the monetary front, following the technical training provided to pilot commercial banks, the central bank launched repo operations (April 2011), the development of which was impeded by the decrease in bank liquidity in the last six months. As part of the financial sector reform, the central bank plans to strengthen the financial infrastructure by computerizing its activities and modernizing its payment system (RTGS and electronic clearing). Competitive bidding for modernization of the computing room has ended; the three firms selected have already submitted their proposals, which are being analyzed. Preparations for the implementation of a modern payment system are under way and the Draft Law Establishing the National Payment System has 
been completed and forwarded to the Council of Ministers for adoption. The strategic and conceptual study on electronic money has been completed and the legal frameworks for e-money and mobile banking are being prepared. Studies on the establishment of a complete clearing and settlement infrastructure have also been concluded and the respective systems will be put in place before a unit responsible for payment systems operation and oversight is established. The financial market development plan was finalized in March 2012 and submitted for review by the Council of Ministers.

12. In the field of banking supervision, business continuity directives have been issued to the banking sector and a detailed manual and a draft circular on risk-based supervision will be prepared with Central AFRITAC technical assistance. In addition, the banking supervision service was restructured to include the function of financial stability assessment. The central bank strengthened its internal control and risk management system with the establishment of an Audit Committee and a revised audit charter conforming to international standards. A risk-based approach was also adopted. To ensure that the regulatory framework favors the development of microfinance institutions, a revised draft decree regulating the microfinance sector is nearing completion and is currently being analyzed by the Bank's management. With regard to banking supervision, a new draft banking law was prepared with IMF technical assistance and the commercial banks will migrate to the IFRS (International Financial Reporting Standards) by end-2013. A new commercial bank, Kenya Commercial Bank, has opened a branch in Burundi.

13. In the coffee sector, the government successfully resumed its washing stations privatization program, with 28 stations sold to Burundian private investors, and made certain that coffee growers received their reserved share in keeping with the government's commitment (25 percent of the capital). A new invitation to bid will be issued for the remaining 76 washing stations no later than end-2012. The government recognizes the preponderant role that the private sector should play in the coffee sector, as part of its strategy to boost production and minimize the cyclical effects of coffee production.

14. With respect to governance, the government has made substantial progress in implementing the national good governance and anti-corruption strategy. The outcome in the INTERPETROL case was a Supreme Court ruling in INTERPETROL's favor. The first review of implementation of the good governance strategy revealed that effective implementation would require the establishment of a technical secretariat.

15. The government has made strides in improving the business climate to attract investment. The reforms carried out had a direct impact on four indicators: (i) business start-ups; (ii) the issuance 
of building permits; (iii) property transfers; and (iv) the protection of investors. These reforms helped improve Burundi's ranking, making it one of the ten best reforming countries in the world.

\section{ECONOMIC OUTLOOK AND POLICIES FOR 2012}

16. The economic and financial program for 2012 is consistent with the three-year ECFsupported economic program. It aims to consolidate gains, particularly with respect to macroeconomic stability and strong, sustained economic growth. However, because of persistent oil and food price shocks and the decline in donor support, which add to the uncertainty of the shortand medium-term economic outlook, the macroeconomic framework for 2012 has been revised as follows: (i) economic growth is expected to level off at 4.2 percent, which is below our initial forecasts; (ii) inflation at end-2012 is estimated at 14.7 percent thanks to a more restrictive monetary policy, increased food production, and a decrease in international oil and food prices; and (iii) official reserves are expected to stabilize at 3.8 months of imports. Moreover, in response to the impact of external shocks on the budget, the government will continue its fiscal consolidation efforts and take steps to expand the tax base and implement a fiscal policy designed to control expenditure.

17. Since the start of the year, the national and international economic context has profoundly affected execution of the 2012 budget. Because of the persistence of relatively high food and oil prices, the government implemented tax relief measures aimed at easing social tensions. The elimination of taxes on food led to a drop in revenue estimated at FBu 10 billion. Moreover, oilrelated revenues are expected to shrink by approximately FBu 23 billion, owing to the reduction of excise taxes on oil products in conjunction with the rise in international oil prices and partial pass through to domestic fuel prices. Expenditure projections point to an overrun of about FBu 18 billion, essentially reflecting the increase in the wage bill, attributable to the lack of control over hiring and the payment of wage arrears and other outlays in the health sector.

18. The government plans to boost revenue collection and to control expenditure in order to close the financing gap caused by expenditure overruns and the elimination of taxes. Consequently, the government adopted the following new tax measures in the context of revising the 2012 Budget Law: (i) replacement of the existing ad valorem tax on beer with an excise tax expressed in terms of hectoliters of beer sold ( $\mathrm{FBu} 30,000 / \mathrm{HI})$; (ii) institution of a 10 percent tax on telephone communications; (iii) increase of the VAT levied on alcoholic beverages (other than beer) from 50 percent to 70 percent; (iv) implementation of a new assessment system for the tax on used vehicles (v) increase of excise taxes on tobacco products; and (vi) a 10 percent income tax rate 
applied to wages of high government official. These measures would generate approximately FBu 26.9 billion and boost tax revenue to FBu 501.2 billion, or 14.1 of GDP.

19. The government is also committed to taking corrective measures on expenditure. Consequently, current expenditure will decrease by 0.5 percent of GDP. Cuts will be mainly in domestically financed capital spending and the wage bill will be contained at 8.1 percent of GDP, with hiring in the health and education sectors remaining a priority. Self-financed capital expenditure is also projected to decrease by FBu 18.4 billion, or 0.5 percent of GDP.

20. These efforts will bring the projected budget deficit into line with the program objectives. The overall 2012 budget deficit (including grants) is estimated at 2.7 of GDP. The financing gap will be covered by domestic borrowing without any risk to debt sustainability. All these changes will be included in a supplementary budget.

21. The Government will continue the reinforcement of safeguards. In this respect, it will ensure that the recruitment of the international auditor takes place before mid-July 2012 to (i) supervise the full execution of all the recommendations made in the report GPO Deloitte 2011; (ii) control on a test basis significant domestic disbursements and transfers on behalf of the Government or in that of its creditors who took place during the period going from July 2011 to March 2012, and (iii) evaluate the state of execution of the new decree on the management of public expenditure, which replaces the General Payment of Public accounts (RGCP) regulation of 1964 . The final audit report will be made available by end-September 2012. The delay observed in the recruitment of the auditor is partly attributable to the need for taking into account the suggestions of the March 2012 IMF safeguards assessment mission which help developing terms of reference for the audit.

\section{Promotion of Good Governance}

22. In the health sector, the government will continue the policy of providing free healthcare for children under 5 years of age and covering the costs of childbirth. Additional infrastructure investments are planned to meet the growing demand for health services. The government will continue to give priority to the hiring of medical personnel in a context of wage bill stabilization. These measures will contribute to higher quality medical care.

23. In the education sector, the government also intends to continue the program of free primary school tuition and will expand it to include higher education. It plans to build new classrooms and hire teachers in order to reduce the teacher-student ratio. 
24. In the agricultural sector, the government, in close collaboration with donors, plans to combat the high cost of living and eliminate food insecurity. Accordingly, the government will implement the National Agricultural and Livestock Investment Plan, the priorities of which are as follows: (i) sustainable growth of production and food security; (ii) professionalization of producers and promotion of innovation; (iii) development of industries and agribusiness, including livestock and fishery activities; and (iv) institution building.

\section{A. Structural reforms}

25. Public financial management reform remains at the center of the government's economic reform policy. Accordingly, texts implementing the decree setting out the general regulation on government budget management will be issued in the coming months, thanks to the technical assistance provided by our development partners. For example, the decree on fiscal governance was reviewed by the Council of Ministers and will be adopted by mid July 2012 after ensuring that the observations of the Council of Ministers are reflected the final text. The assignment of expenditure commitment auditors to two pilot ministries will occur shortly thereafter. The decree on the appointment and legal status of the revenue office's receiver general was examined by the Council of Ministers. This decree defines the function of the OBR receiver general and determines the scope of his authority and his relations with the senior government accountant. The OBR commissionergeneral also fulfills the functions the Office's receiver general. The implementation of a new organizational chart is also planned for the Ministry of Finance and Economic Development Planning (MFPDE), the broad outlines of which were discussed with the IMF. The purpose of the reorganization of the MFPDE is to provide the ministry with the procedures and tools necessary to successfully implement the new fiscal, financial, and accounting regulatory framework adopted in application of the organic law on government finance. In addition, to consolidate the gains achieved in the area of public financial management as a result of implementation of the first public financial management strategy (PFMS-1), as well as the 2009-11 action plan, the government intends to formulate a new public financial management strategy based on the results of the PEFA (Public Expenditure and Financial Accountability) assessment. This new strategy is aimed at increasing the effectiveness of the basic functions of public financial management, in particular by finalizing a consistent legal and institutional framework for public financial management, efficiently mobilizing domestic and external resources, and enhancing the efficiency, accuracy, and transparency of the processes of preparing and executing the government budget.

26. Debt sustainability remains one of the top priorities of the program, given the small range of Burundi's exports and its limited repayment capacity. The Debt Management Performance Assessment (DEMPA) recently carried out by the World Bank revealed that the structure of our debt, 
while sustainable, remains vulnerable to exogenous shocks. The risk of over indebtedness is high. Consequently, in financing economic development, the government will only seek grants or highly concessional loans having a grant element of at least 50 percent. A World Bank (DEMPA) mission is also planned to develop an action plan aimed at (i) improving the legal framework governing the issuance of guarantees and onlending; (ii) strengthening the debt management structure; and (iii) building capacity for the analysis of cost indicators and basic debt risks. With a view to improving debt management, a program to build debt management capacity is being carried out by UNCTAD with financing from the European Union. The program also includes the development and installation of a new version of the SYGADE software $(6.0$,$) an update of the debt database, and the$ publication of a debt statistics bulletin beginning in July 2012.

27. As part of the financial sector reform, the Ministry of Finance, in collaboration with the central bank, plans to establish a unit to coordinate and monitor the reform strategy and the action plan. The responsibilities of this unit will be to: (i) monitor and assess implementation of the strategy and the action plan, by preparing, in advance, performance indicators and measures for verifying results; (ii) serve as the interface between the institutional actors in the sector and as liaison with donors; (iii) assist the authorities in seeking financing and mobilizing resources; (iv) carry out an information and awareness campaign to ensure coverage of the reforms in the media and other publications. The unit will be supervised by a steering committee, which will be responsible, inter alia, for crafting broad guidelines for the conduct of the strategy and for implementation of the payment system computerization projects.

28. To facilitate expanded access to formal financial services by the poor, a national survey on financial inclusion is under way. It will help the central bank better understand the demand for financial services, gauge the degree of satisfaction of the public, develop strategies to expand access to financial services geared to the various needs of the poor and increase the use and quality of such services.

29. In the energy sector, the government plans to pursue its policy aimed at supplying more of the country with electricity and improving the financial position of REGIDESO (public utility Company). The government intends to promote private sector participation in the power generation and distribution sector within the framework of a public-private partnership. Consequently, in the next four years, the government plans to increase power generation capacity by $157 \mathrm{MW}$ with the implementation of subregional hydroelectric dam projects such as Rusumo Falls (60MW) and Ruzizi (48.3MW) and domestic investments such as Kabu 16. These hyrdroelectric plants will be constructed through the European Development Fund, the World Bank, and Exim Bank of India. REGIDESO's financial position was significantly improved by the raising of electricity and water rates 
and the collection of large consumers' bills thanks to the introduction of a prepayment system, which will be expanded with a view to solidifying the improvement in REGIDESO's finances.

\section{Poverty Reduction and Growth Strategy Paper}

30. The Poverty Reduction and Growth Strategy Paper (PRSP-II) was adopted by the government in December 2011 and forwarded to the World Bank and the International Monetary Fund following intensive participatory consultations including representatives of the government, grassroots communities, donors, civil society, the private sector, and Parliament. The strategy was officially launched by the President of the Republic on February 9, 2012. To increase awareness among its development partners, government delegations visited several capitals and headquarters of international institutions. The priority action plan for implementation of the PRSP-II is being finalized, while the government, in concert with its development partners, is in the process of preparing a donor conference, scheduled for October 2012.

31. The PRSP-II, which is a key component of the effort to consolidate peace and kick-start economic growth, is structured around four strategic pillars:

- Strengthening of the rule of law, consolidation of good governance, and promotion of gender equality;

- Transformation of the Burundian economy to achieve sustained, job-creating growth;

- Improvement in the accessibility and quality of basic services and strengthening of national solidarity; and

- Management of land and the environment in harmony with sustainable development principles.

32. The PRSP-II aims to promote strong economic growth which is anchored on a transformation of the economy, for which the financing would come from internal revenue and grants. According to this scenario, growth would average 6.9 percent over 2012-15, without comprising fiscal and debt sustainability. Conversely, the government stands ready to enact measures that will tighten fiscal and monetary policies should budget support grants be lower than expected. These measures will be taken in conjunction with the IMF.

33. With a view to strengthening the ownership and accountability of all stakeholders, meetings to disseminate the contents of the PRSP-II will be held throughout the country, beginning in July. The development of a communications strategy will be an integral part of that stage, to better prepare for the involvement of all participants in the preparatory phase of the first review of PRSP-II implementation. 


\section{A. Improvement of statistics}

34. To lay the foundations of an effective system for monitoring socioeconomic progress in Burundi, the government will pursue efforts to improve the statistical system by strengthening the units in charge of collecting data for the regular production of high quality economic and financial data.

35. To strengthen the statistical services of the sectoral ministries, a draft decree on the creation, organization, and composition of the central ministries' statistical services will be submitted to the Council of Ministers for adoption in the latter half of 2012. To make up for the gaps in statistical data, the ISTEEBU plans shortly to begin conducting surveys on (i) the living conditions of Burundian households; (ii) the work force in Burundi; and (iii) malaria indicators in Burundi. The household survey on living conditions will also serve as the basis for redefining the CPI basket. To this end, the government will request IMF technical assistance to help strengthen its statistical capacity.

\section{PROGRAM MONITORING}

36. Quarterly monitoring of the program by the IMF Executive Board will continue, based on the quantitative monitoring indicators and structural benchmarks described in Tables I.1 and I.2. These indicators are defined in the attached Technical Memorandum of Understanding (TMU). The semiannual reviews will be based on the data at end-March and end-September. The second program review will be based on the performance criteria for end-September. To ensure the success of the program, the authorities will take all steps necessary to achieve the quantitative targets and structural benchmarks agreed with IMF staff. 
Table I.1. Performance Criteria and Indicative Targets for 2012

\begin{tabular}{|c|c|c|c|c|c|c|c|c|c|c|c|c|c|}
\hline & \multirow{2}{*}{\multicolumn{4}{|c|}{$\frac{2011}{\text { Dec. }^{1}}$}} & \multicolumn{9}{|c|}{2012} \\
\hline & & & & & \multicolumn{4}{|c|}{ Mar. } & Jun. ${ }^{1}$ & \multicolumn{2}{|c|}{ Sep. } & \multicolumn{2}{|c|}{ Dec. $^{1}$} \\
\hline & $\begin{array}{l}\text { Rev. } \\
\text { Prog. }\end{array}$ & $\begin{aligned} & \text { Prog. } \\
& \text { Adj. }\end{aligned}$ & Act. & Status & Prog. & $\begin{aligned} \text { Prog. } & \text { Adj. }\end{aligned}$ & Act. & Status & Prog. & Prog. & $\begin{array}{l}\text { Rev. } \\
\text { Prog. }\end{array}$ & Prog. & $\begin{array}{l}\text { Rev. } \\
\text { Prog. }\end{array}$ \\
\hline \multicolumn{14}{|l|}{ Performance criteria } \\
\hline Net foreign assets of the BRB (cumulative floor; US $\$$ million) ${ }^{2}$ & 70.3 & 16.9 & 19.2 & Met & 17.1 & 8.1 & 12.1 & Met & 35.3 & 18.5 & 11.1 & 59.3 & 10.1 \\
\hline Net domestic assets of the BRB (cummulative ceiling) ${ }^{2}$ & 206.1 & 213.3 & 185.1 & Met & 187.3 & 199.1 & 176.5 & Met & 198.7 & 223.9 & 235.0 & 187.2 & 234.8 \\
\hline Net domestic financing of the government (cummulative ceiling) ${ }^{2}$ & 46.6 & 116.3 & 69.8 & Met & 25.7 & 37.5 & -38.0 & Met & 61.8 & 104.1 & 49.0 & 59.5 & 34.6 \\
\hline External payments arrears of the government (ceiling; US $\$$ million) ${ }^{3}$ & 0.0 & 0.0 & 0.0 & Met & 0.0 & 0.0 & 0.0 & Met & 0.0 & 0.0 & 0.0 & 0.0 & 0.0 \\
\hline $\begin{array}{l}\text { Short-term external debt of the government (ceiling; US } \$ \text { million) })^{3,4} \\
\text { Nonconcessional external debt contracted or quaranteed by the }\end{array}$ & 0.0 & 0.0 & 0.0 & Met & 0.0 & 0.0 & 0.0 & Met & 0.0 & 0.0 & 0.0 & 0.0 & 0.0 \\
\hline government or the BRB (ceiling; US\$ million, cumulative from beginning of calendar year) ${ }^{3.4}$ & 80.0 & 80.0 & 80.0 & Met & 0.0 & 0.0 & 0.0 & Met & 0.0 & 0.0 & 0.0 & 0.0 & 0.0 \\
\hline \multicolumn{14}{|l|}{ Indicative targets } \\
\hline Accumulation of domestic arrears (ceiling; cumulative from beginning of calendar year) & 0.0 & & 0.0 & Met & 0.0 & 0.0 & 0.0 & Met & 0.0 & 0.0 & 0.0 & 0.0 & 0.0 \\
\hline Reserve money (ceiling) & 235.5 & & 211.2 & Met & 209.7 & 0.0 & 193.4 & Met & 245.4 & 248.6 & 250.7 & 266.8 & 249.0 \\
\hline Pro-poor spending (floor; cumulative from beginning of calendar year) & 372.6 & & 371.5 & Not Met & 42.0 & 0.0 & 70.1 & Met & 144.7 & 230.6 & 288.3 & 370.1 & 398.0 \\
\hline \multicolumn{14}{|l|}{ Memorandum item: } \\
\hline External nonproject financial assistance (US\$ million; cumulative from beginning of calendar year) & 128.6 & & 91.8 & & 9.0 & 0.0 & 0.0 & & 16.6 & 21.4 & 55.8 & 66.0 & 80.8 \\
\hline 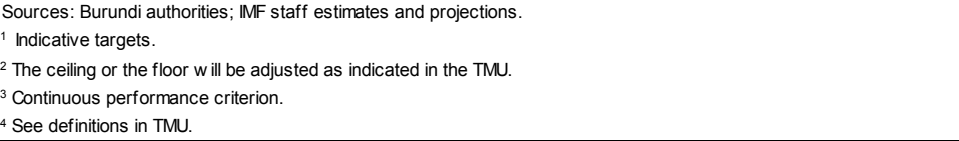 & & & & & & & & & & & & & \\
\hline
\end{tabular}


Table I.2. Structural Benchmarks for 2012

Proposed Measures

\section{Public Financial Management}

Adopt decree on budget governance.

Deploy expenditure commitment controllers in the ministries of health and agriculture.

Approve plan for reorganization of Ministry of Finance and Plan.

\section{Tax Policy}

Prepare a report aimed at: i) identifying all codes and other legal texts in which tax exemptions need to be removed in the context of passing the new income tax law ii) quantifying foregone revenue by tax exemptions in current legislation consistent with Fund TA.

\section{Debt Management}

Undertake an assessment of debt management by the World Bank and develop an action plan to improve capacity.

\section{Central Bank and Treasury Safeguard measures}

Recruit an international auditor to: (i) monitor the full implementation of all the recommendations formulated in GPO

Deloitte's 2011 special audit report;

(ii) verify on a test basis the controls on significant domestic disbursements and transfers-on behalf of the government or its creditors-that took place in the period from July 2011 through March 2012, and (iii) evaluate the implementation of the new decree on management of public expenditures that was approved to replace the 1964 RGCP.

Submit to the General Council, the audit committee, and the Minister of Finance the report on special audits of the controls on important domestic disbursements and transfers-on behalf of the government or its creditors - that took place in the period from July 2011 through March 2012.
June 30, 2012

September 30, 2012

September 30, 2012

September 30 2012

September 30, 2012

March 31, 2012

Delayed pending incorporation of safeguards recommendations in the TOR. Awaiting Presidential signature.
Evaluation done in April 2012, Action plan being developed.

Will facilitate realigning current budget law.

Improve budget discipline in line ministries.

The current allocation of administrative resources is not wellsuited for medium-term reforms included in the budget organic law (i.e. program budgeting and mediumterm programming).

Quantify the fiscal cost of tax exemptions in current legislation. Ensure consistency of investment incentive policy across legislation. Align tax exemptions with other EAC countries and minimize revenue loses.

\section{Improve debt management capacity of the Finance ministry.}

To enhance the safeguard measures in force at the Central Bank and the Treasury.
June 30, 2012 Delayed pending the recruitment of the international auditor.
To enhance the safeguard measures in force at the Central Bank and the Treasury. 


\section{ATTACHMENT II. TECHNICAL MEMORANDUM OF UNDERSTANDING}

Bujumbura, July 9, 2012

1. This technical memorandum of understanding covers the agreements on monitoring implementation of the program supported by the Extended Credit Facility (ECF) Arrangement. It sets out the definitions of program variables to monitor implementation of the program and the reporting requirements for the government of Burundi and the Bank of the Republic of Burundi (BRB). It defines quantitative performance criteria, indicative targets, and applicable adjusters.

\section{A. Quantitative Program Targets}

\section{Quantitative performance criteria and indicative targets}

2. The quantitative performance criteria for the program as shown in the MEFP are as follows:

- $\quad$ net foreign assets of the BRB (floor);

- net domestic assets of the BRB (ceiling);

- net domestic financing of the government (ceiling);

- external payment arrears of the government (ceiling, continuous);

- stock of short-term external debt (maturity of less than one year) of the government (ceiling, continuous); and

- new nonconcessional medium- and long-term external debt contracted or guaranteed by the government or the BRB (ceiling, continuous).

3. The quantitative indicative targets for the program, shown in the MEFP, are as follows:

- accumulation of domestic arrears (ceiling);

- pro-poor spending (floor); and

- reserve money (ceiling).

\section{Definitions and measurement}

4. The net foreign assets of the BRB are defined as the difference between (i) gross official reserves (valued at market prices) and other claims; and (ii) foreign exchange liabilities to nonresident entities (including the use of Fund resources, and liabilities arising from the use of any SDR 
allocation). The gross official reserves of the BRB are defined as those foreign assets that are liquid and freely available to the BRB.

5. The net domestic assets of the BRB are defined as the difference between (i) reserve money, comprising currency in circulation, reserves of commercial banks, and other deposits held at the $B R B$; and (ii) net foreign assets of the BRB.

\section{Adjuster for changes in the compulsory reserves coefficients}

6. The ceiling on net domestic assets of the BRB will be adjusted symmetrically for any change in the compulsory reserves coefficient applied to deposits in commercial banks by the amount of the new coefficient minus that stipulated in the program, multiplied by bank deposits subject to compulsory reserves. The rate stipulated in the program is currently 3 percent.

7. Net domestic financing of the government is defined as the change in (i) outstanding loans, advances, and other credit to the government from the BRB and all of Burundi's commercial banks; (ii) plus the stock of all government securities held by the nonbank public denominated in Burundi francs, including that held by nonresidents; (iii) less government deposits held in the BRB or in Burundi's commercial banks. The coverage of government is defined as central government and any other special funds or operations that are part of the budgetary process or have a direct impact on the government's financial position.

8. The stock of external payment arrears of the government for program monitoring purposes is defined as the end-of-period amount of external debt service due and not paid within the grace period defined by a creditor, including contractual and late interest, for which a clearance agreement is not in place or for which arrears are not rescheduable. For arrears to exist, a creditor must claim payment of amounts due and not paid. Amounts in dispute are not considered arrears. Arrears for which a clearance framework has been agreed with the creditor or which are subject to rescheduling or restructuring are not considered arrears for program monitoring purposes. Program arrears would include any debt service due under such agreements that have not been paid. 


\section{Definition of debt}

9. The program includes a ceiling on new nonconcessional external debt contracted or guaranteed by the government or the BRB. For the purpose of this program, external debt is defined as all debt contracted in a currency other than the Burundian Franc. This performance criterion applies to the contracting or guaranteeing by the government, local governments, the BRB and REGIDESO of new nonconcessional external debt (as specified below) with an original maturity of one year or more, including commitments contracted or guaranteed for which value has not been received (including leases). The coverage of government is defined as central government and any other special funds or operations that are part of the budgetary process or have a direct impact on the government's financial position. Debt contracted by state-owned enterprises is included in the overall ceiling, if guaranteed by the government.

10. For program purposes, the definition of debt is set out in Executive Board Decision No. 12274, Point 9, as revised on August 31, 2009 (Decision No. 14416-(09/91)).

a) For the purpose of this guideline, the term "debt" will be understood to mean a current, i.e., not contingent, liability, created under a contractual arrangement through the provision of value in the form of assets (including currency) or services, and which requires the obligor to make one or more payments in the form of assets (including currency) or services, at some future point(s) in time; these payments will discharge the principal and/or interest liabilities incurred under the contract. Debts can take a number of forms, the primary ones being as follows:

(i) loans, i.e., advances of money to the obligor by the lender made on the basis of an undertaking that the obligor will repay the funds in the future (including deposits, bonds, debentures, commercial loans and buyers' credits) and temporary exchanges of assets that are equivalent to fully collateralized loans under which the obligor is required to repay the funds, and usually pay interest, by repurchasing the collateral from the buyer in the future (such as repurchase agreements and official swap arrangements);

(ii) suppliers' credits, i.e., contracts where the supplier permits the obligor to defer payments until sometime after the date on which the goods are delivered or services are provided; and 
(iii) leases, i.e., arrangements under which property is provided which the lessee has the right to use for one or more specified period(s) of time that are usually shorter than the total expected service life of the property, while the lessor retains the title to the property. For the purpose of the guideline, the debt is the present value (at the inception of the lease) of all lease payments expected to be made during the period of the agreement excluding those payments that cover the operation, repair or maintenance of the property.

b) Under the definition of debt set out in point 9 (a) above, arrears, penalties, and judicially awarded damages arising from the failure to make payment under a contractual obligation that constitutes debt are debt. Failure to make payment on an obligation that is not considered debt under this definition (e.g., payment on delivery) will not give rise to debt.

11. The grant element of debt is the difference between the present value (PV) of debt and its nominal value, expressed as a percentage of the nominal value of the debt. The PV of debt at the time of contracting is calculated by discounting the future stream of payments of debt service due on this debt. The calculation of concessionality will take into account all aspects of the loan agreement, including maturity, grace period, payment schedule, upfront commissions, and management fees. For loans with a maturity of at least 15 years, the 10-year average commercial interest reference rates (CIRRs) published by the OECD should be used as the discount rate for assessing the level of concessionality, while the 6-month average CIRRs should be used for loans with shorter maturities. To both the 10-year and the 6-month average CIRRs, the following margins should be added: 0.75 percent for repayment periods of less than 15 years; 1 percent for 15-19 years; 1.15 percent for 20-29 years; and 1.25 percent for 30 years or more. The performance criterion is defined to exclude rescheduling arrangements, borrowings from the IMF and any Burundi franc-denominated treasury securities held by nonresidents.

12. The stock of short-term external debt with a maturity of less than one year owed by the government is to remain at zero under the program. The coverage of government is defined as central government and any other special funds or operations that are part of the budgetary process or have a direct impact on the government's financial position. Normal import credits are excluded from this ceiling. Loans with an initial maturity, as recorded in the original loan agreement, of one year or more are considered medium-term or long-term loans. This performance criterion applies not only to debt as defined in point No. 9 of the Guidelines on 
Performance Criteria with Respect to Foreign Debt, adopted August 24, 2000, but also to commitments contracted or guaranteed for which value has not been received (including leases). Excluded from this performance criterion are rescheduling arrangements, borrowing from the IMF, and any Burundi franc-denominated treasury securities held by nonresidents. As of September 2007, the stock of short-term debt outstanding was nil.

13. Consistent with the PRSP, the authorities' definition of pro-poor spending is based on three criteria: (i) social character of spending, based on the administrative classification of spending (this includes "social services" spending and part of "general services" and "economic services" spending if it has a social character component); (ii) consistency with one of the four PRSP pillars; and (iii) pro-poor investment spending, financed by donors.

14. The accumulation of domestic arrears is measured by the accumulation of non-executed payment orders older than 60 days.

\section{External financial assistance adjustor}

15. The program provides for adjusters to allow higher than expected external assistance to be spent (with a cap) and shortfall of external assistance to be financed domestically (with a cap).

16. Any financing excess up to US $\$ 40$ million will be spent on expenditure priorities defined in the PRSP. The floor on the stock of net foreign assets of the BRB will be adjusted upward, and the ceilings on the net domestic assets of the BRB and on net domestic financing to the government will be adjusted downward to accommodate 100 percent of any financing excess above US\$40 million.

17. The floor on the stock of net foreign assets of the BRB will be adjusted downward, and the ceilings on the net domestic assets of the BRB and on net domestic financing to the government will be adjusted upward to accommodate a financing shortfall up to a maximum of US $\$ 40$ million. External financial assistance will be converted to Burundi francs using the program-specified BIF/US\$ exchange rate. The program exchange rate for end-June 2012 is 1408.2 and at end-September 2012 is 1416.4 .

18. External financial assistance (measured in US\$) is defined to include the following: (i) nonproject loans and grants to the budget (including payments made through the multi-donor trust fund 
managed by the World Bank for current debt service to multilaterals); plus (ii) debt relief on current maturities; minus (iii) any cash payments for external arrears clearance operations. Donor disbursements into blocked accounts for the purpose of clearing arrears will not be considered foreign assistance for program monitoring purposes.

\section{B. Provision Of INFORMATION to IMF StafF}

19. To facilitate the monitoring of program implementation, the authorities will prepare and forward to the IMF African Department a monthly progress report on the program, within six weeks of the end of each month, containing the following weekly data:

- foreign exchange auction market (MESD) transactions;

- the balance sheet of the BRB (weekly statement) (BRB Research Department).

20. The following monthly data, with a maximum lag of six weeks:

- a monitoring table (tableau de bord) containing the most recent weekly and monthly data on the main financial indicators (REFES);

- a table on foreign exchange cash flow (BRB Foreign Banking Operations Department);

- the monetary survey, including the breakdown of the BRB and of commercial banks (BRB Research Department);

- monthly exchange-rate data (official and parallel markets, end-of-month and monthly average) (BRB Research Department);

- a detailed breakdown of government revenue (Ministry of Finance);

- a detailed breakdown of government expenditure on a commitment basis, including pro-poor spending (Ministry of Finance);

- a detailed breakdown of the servicing of domestic and external public debt, including amounts due and paid, on interest and principal, as well as the breakdown by creditor and any accumulation of arrears on domestic or external debt (Ministry of Finance);

- a detailed breakdown of the stock of domestic payment arrears for the current fiscal year (Ministry of Finance);

- the amount of new debts contracted or guaranteed by the government, including 
detailed information on the terms (such as currency denomination, interest rate, grace period, maturity) (Ministry of Finance);

- actual disbursements of nonproject financial assistance, including new loans and debt relief granted by Burundi's external creditors (Ministry of Finance); and

- an update on the implementation of structural measures planned under the program (REFES).

21. The following quarterly data, with a maximum lag of six weeks:

- $\quad$ progress reports on the BRB's internal reforms, including each unit's action plans for the coming month (Reform Monitoring Committee, BRB).

22. SP/REFES/Ministry of Finance and BRB will also provide the IMF African Department with any information that is deemed necessary to ensure effective monitoring of the program. 


\title{
INTERNATIONAL MONETARY FUND
}

\section{BURUNDI}

STAFF REPORT FOR THE 2012 ARTICLE IV CONSULTATION AND FIRST REVIEW UNDER THE THREE-YEAR

July 12, 2012 ARRANGEMENT OF THE EXTENDED CREDIT FACILITY AND REQUEST FOR MODIFICATION OF PERFORMANCE CRITERIA — INFORMATIONAL ANNEX

\author{
Prepared By \\ The African Department
}

\section{CONTENTS}

RELATIONS WITH THE FUND 74

JOINT WORLD BANK-IMF WORK PROGRAM, 2011-12 80

RELATIONS WITH THE AFRICAN DEVELOPMENT BANK GROUD 82 


\section{RELATIONS WITH THE FUND}

(As of May $31^{\text {st }}, 2012$ )

I. Membership Status: Joined: September 28, 1963;

$\underline{\text { Article XIV }}$

II. General Resources Account:

Quota

SDR Million

77.00

76.64

0.36

Reserve Tranche Position

III. SDR Department:

Net cumulative allocation

Holdings

IV. Outstanding Purchases and Loans:

ECF Arrangements

V. Latest Financial Arrangements:

\begin{tabular}{|c|c|c|}
\hline Type & $\begin{array}{c}\text { Date of } \\
\text { Arrangement } \\
\end{array}$ & $\begin{array}{c}\text { Expiration } \\
\text { Date }\end{array}$ \\
\hline ECF & Jan 27, 2012 & $\operatorname{Jan} 26,2015$ \\
\hline $\mathrm{ECF}^{1}$ & Jul 07, 2008 & Jan 23, 2012 \\
\hline $\mathrm{ECF}^{1}$ & Jan 23, 2004 & Jan 22, 2008 \\
\hline
\end{tabular}

SDR Million

73.85

82.87

SDR Million

86.82

Amount Approved

(SDR Million)

30.00

51.20

69.30
\%Quota

100.00

99.53

0.47

\%Allocation

100.00

112.22

\%Quota

112.75

Amount Drawn (SDR Million)

1.00

51.20

69.30

VI. Projected Payments to Fund ${ }^{2}$

(SDR Million; based on existing use of resources and present holdings of SDRs):

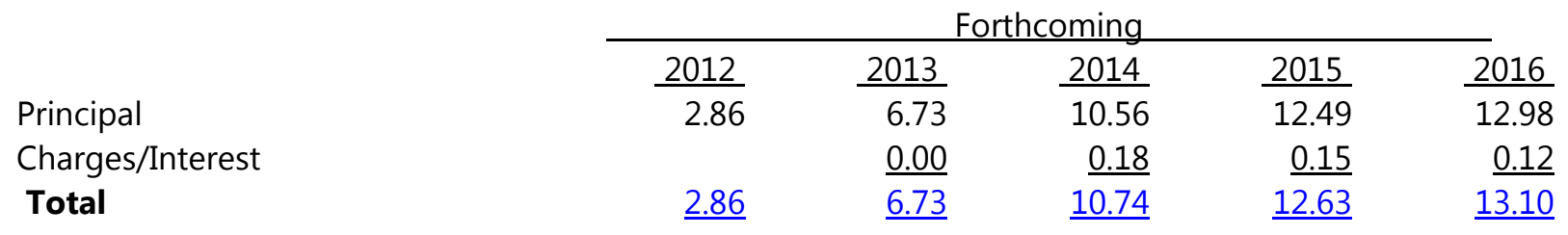

\footnotetext{
1 Formerly PRGF.

${ }^{2}$ When a member has overdue financial obligations outstanding for more than three months, the amount of such arrears will be shown in this section.
} 
VII. Implementation of HIPC Initiative:

I. Commitment of HIPC assistance

Assistance committed

by all creditors (US\$ Million) ${ }^{3}$

Of which: IMF assistance (US\$ million)

(SDR equivalent in millions)

Completion point date

II. Disbursement of IMF assistance (SDR Million)

Assistance disbursed to the member

Interim assistance

Completion point balance

Additional disbursement of interest income ${ }^{4}$

Total disbursements

VIII. Implementation of Multilateral Debt Relief Initiative (MDRI):

I. MDRI-eligible debt (SDR Million) ${ }^{5}$

$\begin{array}{lr}\text { Financed by: MDRI Trust } & 9.01\end{array}$

Remaining HIPC resources 17.39

II. Debt Relief by Facility (SDR Million)

Eligible Debt

\section{Delivery}

Date

GRA

PRGT

$\underline{\text { Total }}$

February 2009

N/A

26.40

26.40

\footnotetext{
${ }^{3}$ Assistance committed under the original framework is expressed in net present value (NPV) terms at the completion point, and assistance committed under the enhanced framework is expressed in NPV terms at the decision point. Hence these two amounts cannot be added.

${ }^{4}$ Under the enhanced framework, an additional disbursement is made at the completion point corresponding to interest income earned on the amount committed at the decision point but not disbursed during the interim period.

${ }^{5}$ The MDRI provides 100 percent debt relief to eligible member countries that qualified for the assistance. Grant assistance from the MDRI Trust and HIPC resources provide debt relief to cover the full stock of debt owed to the Fund as of end-2004 that remains outstanding at the time the member qualifies for such debt relief.
} 
IX. Implementation of Post-Catastrophe Debt Relief (PCDR): Not Applicable

Decision point - point at which the IMF and the World Bank determine whether a country qualifies for assistance under the HIPC Initiative and decide on the amount of assistance to be committed.

Interim assistance - amount disbursed to a country during the period between decision and completion points, up to 20 percent annually and 60 percent in total of the assistance committed at the decision point (or 25 percent and 75 percent, respectively, in exceptional circumstances).

Completion point - point at which a country receives the remaining balance of its assistance committed at the decision point, together with an additional disbursement of interest income as defined in footnote 2 above. The timing of the completion point is linked to the implementation of pre-agreed key structural reforms (i.e., floating completion point).

\section{Exchange Arrangements}

While the de jure exchange rate arrangement is floating, the de facto exchange rate arrangement has been reclassified to other managed arrangement from stabilized arrangement against the dollar, effective July 26, 2011, due to increased flexibility of the Burundi franc. The US dollar is the intervention currency. On June $14^{\text {th }}, 2012$, the official exchange rate was BIF 1412.04 to the US dollar. In 2003 the central bank eliminated most remaining exchange restrictions on current international transactions and delegated authority to commercial banks to approve standard transactions. In early 2004, the surrender requirement was lowered to 50 percent and in early 2005 it was eliminated. The central bank has admitted foreign exchange bureaus to the weekly auctions. Most external arrears to bilateral and multilateral creditors were cleared by the end of 2005. In December 2006 the government published a new foreign exchange regulation, the Foreign Exchange Regulation of 2006 that liberalized access to foreign exchange for current transactions and removed one multiple currency practice. In June 2010, the government replaced the Foreign Exchange Regulation of 2006 with the Exchange Regulation of 2010. The new regulation became operational in July 2010.

Burundi has availed itself of the transitional arrangements of Article XIV since it joined the Fund in 1962 but no longer maintains any exchange restrictions or multiple currency practices that relate to that article. It does have one multiple currency practice that is inconsistent with Article VIII, Section 2(a): the exchange rate used for government 
transactions differ by more than 2 percent from market exchange rates. Burundi maintains certain foreign exchange restrictions for security reasons and has notified the Fund of those restrictions pursuant to Decision 144-(52/51). Burundi modified the 2010 foreign exchange regulation on March 3, 2011. Consequently the two foreign exchange restrictions mentioned in EBS/11/29 Sup. 1 relating to: (i) a tax clearance requirement for certain current international transactions such as payments of moderate amounts for amortization of loans or for depreciation of direct investments by nonresidents and (ii) the limitations on the availability of foreign exchange for the making of payments and transfers for current international transactions based on noncompliance with obligations that are unrelated to such transactions are no longer in place.

\section{Article IV Consultation}

In accordance with Decision No. 14747-(10/96), adopted September 28, 2010, Burundi is on the 24-month Article IV cycle. The 2008 Article IV consultation was completed by the Executive Board on July 23, 2010, (IMF Country Report No. 10/313), along with the fourth review of the ECF arrangement.

In concluding the 2010 consultation, Executive Directors acknowledged the commendable progress that Burundi made in implementing its first ECF-supported program in a difficult post-conflict environment. They agreed that fiscal sustainability in the face of a heavy debt burden will depend on broadening the revenue base and improving the composition of spending while financing the budget though grants and highly concessional external resources. Directors encouraged the authorities to continue their efforts to reform the financial sector by improving banking supervision, and addressing weaknesses in the banking system, notably concentration risk. Directors also saw the need to accelerate structural reforms, especially in the coffee sector, and welcomed Burundi's membership in the East African Community. 


\section{Technical Assistance}

July 2012

April 2012

December 2011

November 2011

October 2011

July 2011

March 2011

January 2011

September 2010

November 2010

Oct 2010

April 2010

November 2009

September 2009

June 2009

April 2009

March 2009

March 2009

March 2009

March 2009

February 2009

February 2009

January 2009

December 2008

December 2008

December 2008

August 2008

July 2008

June 2008

Oct 2008

December 2007

November/December 2007

November 2007
FAD mission on revenue administration

AFRITAC mission on National Accounts

AFRITAC mission on PFM reform

MCM mission of foreign exchange operations

AFRITAC mission on PFM reform

STA mission on Balance of Payments statistics

FAD mission on implementing the organic budget law

MCM mission on Foreign exchange operations

FAD mission on implementing the organic budget law

MCM mission on monetary operations

FAD mission on Tax administration.

MCM mission on Foreign exchange operations.

AFRITAC mission on VAT implementation.

MCM mission on BRB capacity building and public debt management

MCM multitopic mission

FAD mission on implementing the organic budget law

MCM mission on monetary operations

MCM mission on foreign exchange

MCM mission on internal audit

MCM mission on reserve management

AFRITAC mission on banking regulation and supervision

FAD PSIA mission on fuel pricing policy and social protection MCM FSAP mission

AFRITAC mission on fiscal administration

MCM multi-topic mission

MCM mission on internal audit

MCM mission on organizational, human resources, and communication reforms

MCM mission on foreign exchange

AFRITAC mission on banking regulation and supervision

FAD mission for installation of a PFM resident advisor

AFRITAC statistics mission on national accounts and consumer price index

STA monetary and financial statistics mission

AFRITAC mission on building capacity in banking supervision 
November 2007

November 2007

September 2007

September 2007

September 2007

September 2007

July 2007

June 2007
FAD Tax policy mission

STA monetary statistics mission

MCM multitopic mission

AFRITAC mission on tax revenue administration

AFRITAC mission on building the capacity of the central bank

FAD public financial management mission

AFRITAC PFM mission on payroll control

AFRITAC PFM mission on treasury operation and control

XIII. Implementation of HIPC Initiative:

I. Commitment of HIPC assistance

Decision point date

Assistance committed

by all creditors (US\$ million) ${ }^{6}$

of which: IMF assistance (US\$ million)

(SDR equivalent in millions)

Completion point date

II. Disbursement of IMF assistance (SDR million)

Assistance disbursed to the member

Interim assistance

Completion point balance

Additional disbursement of interest income ${ }^{7}$

Total disbursements
Enhanced

Framework

Aug 2005

832.6

27.87

19.28

Jan. 2009
22.35

\footnotetext{
${ }^{6}$ Assistance committed under the original framework is expressed in net present value (NPV) terms at the completion point, and assistance committed under the enhanced framework is expressed in NPV terms at the decision point. Hence these two amounts cannot be added.

${ }^{7}$ Under the enhanced framework, an additional disbursement is made at the completion point corresponding to interest income earned on the amount committed at the decision point but not disbursed during the interim.
}

\section{Resident Representative}

A part-time resident representative took up the post in Oct 2005 and an office with an administrative assistant opened in January 2006 in Bujumbura. Mr. Koffi Yao has been the resident representative since January 2010. 


\section{JOINT WORLD BANK-IMF WORK PROGRAM, 2011-12}

\begin{tabular}{|c|c|c|c|}
\hline Title & Products & $\begin{array}{c}\text { Provisional Timing of } \\
\text { Mission }\end{array}$ & $\begin{array}{c}\text { Expected Delivery } \\
\text { Date }\end{array}$ \\
\hline
\end{tabular}

\section{A. Mutual Information on Relevant Work Programs}

Bank work program

IMF work program
1. Public Expenditure
Review
2. Public Expenditure
Review Policy Note

\section{ERSG-5 \\ (Budget support)}
4. ERSG-6
(Budget support)
5. Diagnostic Trade Integration Study - Update (DTIS)
1. Seventh ECF review

2. $1^{\text {st }}$ Review of New ECF

3. $2^{\text {nd }}$ review of new ECF

\author{
February 2012 \\ (identification) \\ June 2012 \\ (Main mission)
}

September 2012

May 2012

Dec. 2010 - Jan. 2011

December 2011 (Identification) June 2011

(pre- appraisal) September 2011

(appraisal)

January-February 2012 August 2012 (identification)

April 2012

(pre- appraisal)

May 2012 (appraisal)

November 2011

June 2012

(Identification)

May 2012 (main

mission)

Mar. 2012

November 2012

June 2012

Jan 2012

Nov 2012

July 2012 


\section{B. Requests for Work Program Inputs}

Fund request to Bank

1. Progress report on

March 2012 reform implementation of reforms in REGIDESO

\section{Agreement on Joint Products and Missions}

Joint products JSAN on PRSP

July 2012 


\section{RELATIONS WITH THE AFRICAN DEVELOPMENT BANK GROUP}

(As of May 23, 2012)

Burundi has been a member of the African Development Bank (AfDB) Group since the group was founded in 1964. AfDB grant and loan operations with the country were interrupted by the outbreak of civil strife in 1993. On July 19, 2004, the AfDB Boards approved general policy guidelines to help postconflict countries clear their arrears and created a facility, the PCCF, initially funded with about SDR 100 million in AfDB funds, to provide financial assistance to qualifying countries. The policy guidelines call for a three-way burden-sharing formula among the country, donors, and the PCCF. On October 27, 2004, the AfDB Boards endorsed an arrears clearance proposal for Burundi whereby the balance of arrears was settled with the help of donors and the PCCF before the decision point for the enhanced HIPC Initiative.

On April 21, 2009, under the enhanced framework of the Heavily Indebted Poor Countries (HIPC) Initiative, the Board of Directors of AfDB approved AfDB's share of the HIPC debt relief in an amount equivalent to USD 150,200,000 in Net Present Value (NPV) terms as of the end of 2004. The Board of Directors also approved Burundi's qualification for debt relief under the Multilateral Debt Relief Initiative (MDRI), in an amount of US\$15.38 million, (UA 10.48 million), in nominal terms.

In support of the Government's economic and social program, the Board approved the Country Strategy Paper (CSP) in October 2008. The CSP covers the period 2008 to 2011 and focuses on 2 pillars of the CSLP namely, (i) support to effective government by improving economic governance and the functioning of key public sector institutions, and (ii) increase employment opportunities through developing infrastructure and targeted interventions in the agricultural sector. The Country Strategy Paper Mid-Term Review and Country Portfolio Review have been presented to CODE on $30^{\text {th }}$ of November 2010 and confirmed the relevance of the pillars. The Bank Group intervenes actively in infrastructure (transport, water and sanitation, and energy), agriculture, governance and social sectors. The Bank ongoing portfolio comprises seven (7) national operations for a total UA 112.23 million. The road sector accounts for $58.9 \%$ of the portfolio, followed by the social sector (17.7\%). There are six (6) active multinational operations for UA 117.7 million, mostly in the road sector (70.1\%).The amount of 7 million UA for the fourth phase of Burundi's "Programme d'Appui aux Réformes Economiques" (PARE IV) has been fully disbursed in 2011. 
The 2012-2016 CSP has been approved by the Board of Directors on January $11^{\text {th }}, 2012$. The CSP proposed strategy emphasizes selectivity, with two complementary pillars, namely (i) strengthening state institutions and (ii) infrastructure improvements in order to promote inclusive growth and development. The selection of these pillars is consistent with the Bank's comparative advantage in the infrastructure sector, as well as its value-added in the area of support of reforms, good governance and institutional capacity building. The Bank plans to support Burundi trough infrastructure projects (mainly transport and energy); a two tranches GBS, namely PARE V, amounting of UA 12 million for the period 2012-2013 and capacity building. 


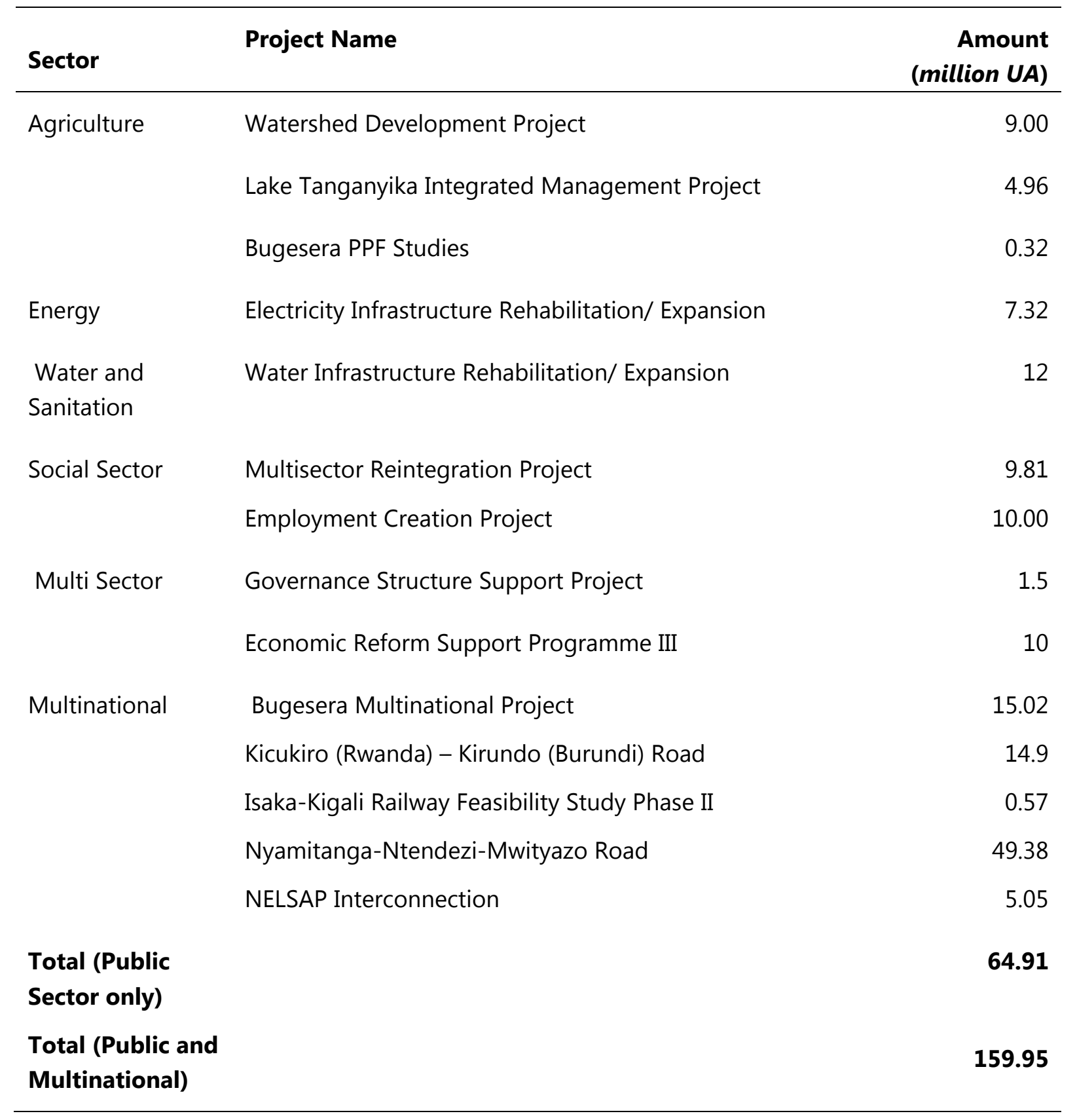




\section{STATISTICAL ISSUES}

(As of June 26, 2012)

\section{Assessment of Data Adequacy for Surveillance}

General: Data provision has serious shortcomings that significantly hamper surveillance. The most serious shortcomings affect national accounts, government finance, and balance of payments statistics. Insufficient funding, staffing shortages and lack of equipment, along with coordination difficulties among responsible institutions, impede the timely production and dissemination of macroeconomic statistics.

Real sector statistics: Serious deficiencies in real sector data compilation hamper economic analysis and management. National accounts statistics are compiled infrequently by ISTEEBU, the national statistical office. Source data on agriculture, the most important economic activity, is inadequate. Consequently, there is a high degree of uncertainty regarding estimates of the level and the growth rate of GDP, components of expenditure, and all ratios to GDP. Annual national accounts estimates provided to the Fund, are not compiled, but rather, are derived from a macroeconomic projection model and certain base year estimates for 1998 and 2005. Since 2007, ISTEEBU has started the process for improvement of national accounts statistics with the assistance of AFRITAC. National accounts are now established on the basis of SNA 1993 (partially) using ERETES software with 2005 as base year. Compilation of GDP estimates for the years 2007 and 2008 is nearly finalized. Data on non-financial corporations is also a major area of concern for national accounts compilers. The monthly consumer price index features expenditure weights derived from a dated 1991 household expenditure survey and covers only the capital city. The CPI also has serious problems with the methodology used, including the lower-level formula, treatment of missing prices, and the sample selection, all of which lead to an inaccurate measurement of inflation. No producer price indices are compiled. Limited labor market statistics are available, thus hampering surveillance.

Government finance statistics: Burundi has benefitted from technical assistance provided by the World Bank and Fiscal Affairs Department in the areas of public financial management information systems and public expenditure management, as well as Statistics Department (STA) training in the compilation of government finance statistics. Nevertheless, government finance statistics (GFS) continue to suffer from weaknesses in coverage, accuracy, consistency, and timeliness. Burundi has accepted to participate in the GFS module under the Enhanced Data Dissemination Initiative (EDDI) project for Africa funded by the United Kingdom Department for International Development (DFID). The GFS module has as its main objective to improve the quality and dissemination of fiscal statistics using the guidelines of the Government Finance Statistics Manual 2001 (GFSM 2001). The support under the module consists mostly of a series of technical assistance missions over the next 3 to 5 years. The first mission took place in April 2011. The second mission will take place later this year.

Monetary and financial statistics: The monetary and financial statistics are now compiled following the recommended methodologies of the IMF's Monetary and Financial Statistics Manual (2000), and are broadly adequate for both surveillance and harmonization with the 
monetary statistics of other East African Community member countries. Transactions with the IMF are reflected incorrectly-the amount of loans received from IMF differs from the IMF records, misrepresenting foreign liabilities of the central bank. STA informed the central bank of the issue, the resolution of which is pending. Because of the current payment system, there are differences between the central bank and other depository corporations (ODC) records. Normally, transactions between the central bank and ODC show up in ODC balance sheet with one-day lag that affects other items (net) (OIN) in the Depository Corporations survey. The liabilities of three banks in liquidation are shown in the central bank balance sheet; however, the information on these banks is not included in the summary of ODC data.

External sector statistics: Annual balance of payments and international investment position statistics are compiled by the Banque de la République du Burundi (BRB) according to the fifth edition of the Balance of Payments Manual (BPM5). However, severe coverage and measurement difficulties impart a high degree of uncertainty to external sector statistics and surveillance. For example, there are significant unrecorded imports and exports (particularly coffee and tea) of merchandise and services, and while adjustments are made, the uncertainty remains. For both services and income, the accuracy of the source data is not routinely assessed against other data sources. Similarly, measurement of capital and financial account transactions relies on Burundi's International Transaction Reporting System, which is known to have incomplete coverage. Further actions are required to improve the accuracy and reliability of external sector data.

\begin{tabular}{|c|c|}
\hline \multicolumn{2}{|c|}{ II. Data Standards and Quality } \\
\hline $\begin{array}{l}\text { Participant in the General Data Dissemination } \\
\text { System (GDDS) since August } 2011 .\end{array}$ & No data ROSC is available. \\
\hline \multicolumn{2}{|c|}{ III. Reporting to STA } \\
\hline $\begin{array}{l}\text { Summary government finance transactions dat } \\
\text { Finance Statistics (IFS). The BRB has completed } \\
\text { the submission of its monetary statistics to the } \\
\text { in the IFS. Balance of payments and internation } \\
\text { and in the Balance of Payments Yearbook. }\end{array}$ & $\begin{array}{l}\text { are reported for publication in International } \\
\text { igration to the Standardized Report Forms for } \\
\text { Ind; detailed monetary statistics are published } \\
\text { investment position data are published in IFS }\end{array}$ \\
\hline
\end{tabular}


Burundi: Table of Common Indicators Required for Surveillance

\begin{tabular}{|c|c|c|c|c|c|}
\hline & $\begin{array}{l}\text { Date of Latest } \\
\text { Observation }\end{array}$ & Date Received & $\begin{array}{l}\text { Frequency of } \\
\text { Data }^{8}\end{array}$ & $\begin{array}{l}\text { Frequency of } \\
\text { Reporting }^{8}\end{array}$ & $\begin{array}{l}\text { Frequency of } \\
\text { Publication }^{8}\end{array}$ \\
\hline $\begin{array}{l}\text { International Reserve Assets and Reserve } \\
\text { Liabilities of the Monetary Authorities }{ }^{1}\end{array}$ & Mar. 2012 & Mar. 2012 & M & M & M \\
\hline Reserve/Base Money & Mar. 2012 & Mar. 2012 & M & M & M \\
\hline Broad Money & Mar. 2012 & Mar. 2012 & M & M & M \\
\hline Central Bank Balance Sheet & Mar. 2012 & Mar. 2012 & M & M & M \\
\hline Consumer Price Index & May 2012 & May 2012 & M & M & M \\
\hline $\begin{array}{l}\text { Revenue, Expenditure, Balance and } \\
\text { Composition of Financing-General } \\
\text { Government }^{4 /}\end{array}$ & NA & NA & NA & NA & NA \\
\hline $\begin{array}{l}\text { Revenue, Expenditure, Balance and } \\
\text { Composition of Financing }{ }^{3}-\text { Central } \\
\text { Government }\end{array}$ & Mar. 2012 & Mar. 2012 & $\mathrm{Q}$ & $\mathrm{Q}$ & $\mathrm{Q}$ \\
\hline GDP/GNP ${ }^{6}$ & 2009 & Mar. 2012 & $A$ & $A$ & $A$ \\
\hline
\end{tabular}


Burundi: Table of Common Indicators Required for Surveillance (concluded)

\begin{tabular}{|c|c|c|c|c|c|}
\hline & $\begin{array}{c}\text { Date of Latest } \\
\text { Observation }\end{array}$ & Date Received & $\begin{array}{c}\text { Frequency of } \\
\text { Data }^{8}\end{array}$ & $\begin{array}{c}\text { Frequency of } \\
\text { Reporting }^{8}\end{array}$ & $\begin{array}{l}\text { Frequency of } \\
\text { Publication }^{8}\end{array}$ \\
\hline Gross External Debt & 2010 & Mar. 2012 & $M$ & M & A \\
\hline International Investment Position ${ }^{7}$ & 2010 & Mar. 2012 & $A$ & $A$ & $A$ \\
\hline
\end{tabular}

\footnotetext{
${ }^{1}$ Any reserve assets that are pledged of otherwise encumbered should be specified separately. Also, data should comprise short-term liabilities linked to a foreign currency but settled by other means, as well as the notional values of financial derivatives to pay and to receive foreign currency, including those linked to a foreign currency but settled by other means.

${ }^{2}$ Both market-based and officially determined, including discount rates, money market rates, rates on treasury bills, notes and bonds.

${ }^{3}$ Foreign, domestic bank, and domestic nonbank financing.

${ }^{4}$ The general government consists of the central government (budgetary funds, extra budgetary funds, and social security funds), and state and local governments.

${ }^{5}$ Including currency and maturity composition.

${ }^{6}$ Last observed data was Dec 2008.

${ }^{7}$ Includes external gross financial asset and liability positions vis-a-vis nonresidents.

${ }^{8}$ Daily $(D)$, weekly $(W)$, monthly $(M)$, quarterly $(\mathrm{Q})$, annually $(A)$, irregular (I); and not available (NA).
} 


\section{INTERNATIONAL MONETARY FUND}

Public Information Notice

EXTERNAL

RELATIONS

DEPARTMENT
Public Information Notice (PIN) No. 12/95

FOR IMMEDIATE RELEASE

August 2, 2012
International Monetary Fund

$70019^{\text {th }}$ Street, NW

Washington, D. C. 20431 USA

\section{IMF Executive Board Concludes 2012 Article IV Consultation with Burundi}

On July 27, 2012, the Executive Board of the International Monetary Fund (IMF) concluded the Article IV consultation with Burundi. ${ }^{1}$

\section{Background}

Real GDP growth is estimated to have increased to 4.2 percent in 2011. After decelerating to 15 percent (y-o-y) toward end-2011, headline inflation rose sharply in March 2012 to 24.5 percent $(y-0-y)$ owing to a rise in rents, utility tariffs, and higher food prices. It has since eased slightly to 22.7 percent in May 2012.

Growth in broad money decelerated to about 4.7 percent (y-o-y) in April 2012 following tighter monetary conditions as policy rates rose by 350 basis points to 14 percent in May 2012. Credit to the private sector after peaking at 40 percent at end-2011 slowed to 28 percent $(y-0-y)$ at end-April 2012 in the face of tighter liquidity conditions. Banks were able to sustain credit growth despite a decline in deposits, in part through a drawdown in excess reserves.

\footnotetext{
${ }^{1}$ Under Article IV of the IMF's Articles of Agreement, the IMF holds bilateral discussions with members, usually every year. A staff team visits the country, collects economic and financial information, and discusses with officials the country's economic developments and policies. On return to headquarters, the staff prepares a report, which forms the basis for discussion by the Executive Board. At the conclusion of the discussion, the Managing Director, as Chairman of the Board, summarizes the views of Executive Directors, and this summary is transmitted to the country's authorities. An explanation of any qualifiers used in summings up can be found here: http://www.imf.org/external/np/sec/misc/qualifiers.htm.
} 
While revenues and expenditure were broadly in line with the program, the deficit in 2011 was higher than expected due to the late disbursement of the World Bank budget support operation. Revenues through end-May 2012 were lower than expected by $(0.5$ percent of GDP), owing to a fall in collections of excise taxes and the granting of exemptions. To limit the impact of inflation on the poor, the government eliminated taxes on food products on May 1 until end-2012.

\section{Executive Board Assessment}

Executive Directors commended the Burundian authorities for progress in implementing their Fund-supported economic program in a difficult post-conflict environment. However, Directors considered that the external and internal risks weighing on the outlook called for a faster pace of fiscal and structural reforms, and encouraged the authorities to persist in their prudent approach to macroeconomic management.

Directors agreed that continued progress in implementing revenue reforms is essential in light of recent fiscal slippages and the decline in donor assistance. In particular, they encouraged the authorities to widen the tax base, strengthen revenue administration, and overhaul the fuel pricing mechanism. Directors commended the authorities for taking sizable corrective fiscal measures to keep the program on track but stressed the need to better align spending priorities in view of resource constraints.

Directors emphasized the importance of pursuing public financial management reforms in order to foster greater transparency and accountability, and to strengthen institutional capacity. They also noted that, despite improvements in the budget framework, significant gaps remain in debt management and Burundi remains vulnerable to debt distress. In this context, continued reliance on grants and highly concessional loans remains a priority.

Directors considered that additional monetary policy tightening will be warranted if inflationary pressures do not subside in the period ahead. They called for further monitoring of risks to the financial sector stemming from rising interest rates and an unfavorable external environment.

Directors acknowledged that greater exchange rate flexibility has helped the economy adjust to external shocks. They underscored the need to further enhance Burundi's competitiveness by strengthening the business climate, improving the electricity supply, and reducing 
transportation costs.

Directors concurred that Burundi's statistical base still suffers from shortcomings that hamper policy design and evaluation. In particular, they encouraged the authorities to improve capacity in the preparation of national accounts, balance of payments data, and consumer price indices.

Public Information Notices (PINs) form part of the IMF's efforts to promote transparency of the IMF's views and analysis of economic developments and policies. With the consent of the country (or countries) concerned, PINs are issued after Executive Board discussions of Article IV consultations with member countries, of its surveillance of developments at the regional level, of post-program monitoring, and of ex post assessments of member countries with longer-term program engagements. PINs are also issued after Executive Board discussions of general policy matters, unless otherwise decided by the Executive Board in a particular case. 
Burundi: Selected Economic and Financial Indicators, 2010-15

\begin{tabular}{lllllll}
\hline 2010 & 2011 & 2012 & 2013 & 2014 & 2015 \\
\cline { 3 - 6 } & & Prel. & & \multicolumn{4}{c}{ Proj. } \\
\hline
\end{tabular}

(Annual percentage change)

National income and prices

$\begin{array}{lrrrrrr}\text { Real GDP growth } & 3.8 & 4.2 & 4.2 & 4.5 & 5.1 & 5.5 \\ \text { GDP deflator } & 12.3 & 14.3 & 15.2 & 11.1 & 7.2 & 5.8 \\ \text { Consumer prices (period average) } & 6.4 & 9.7 & 19.6 & 6.4 & 7.4 & 6.3 \\ \text { Consumer prices (end of period) } & 4.1 & 14.9 & 14.7 & 8.4 & 6.1 & 6.4\end{array}$

External sector

Exports, f.o.b. (US\$)

Imports, f.o.b. (US\$)

Export volume

$\begin{array}{rrrrrr}48.0 & 22.5 & 3.1 & 0.2 & -1.0 & 9.3 \\ 105.3 & 3.7 & -2.9 & 3.5 & 1.2 & 3.7 \\ 16.4 & 0.4 & 30.4 & 5.4 & -3.9 & 14.8 \\ & - & & & & \\ 145.1 & 22.6 & 0.8 & 7.9 & 2.8 & 4.6 \\ & & - & & & \\ 51.7 & -8.9 & 17.9 & -1.0 & 4.6 & -4.0\end{array}$

Terms of trade (deterioration $=-$ )

(Change in percent of beginning of period M2, unless otherwise indicated)

Money and credit

Net foreign assets

Domestic credit Government

Private sector

Money and quasi-money (M2)

Reserve money (12-month growth rate)

General government

Revenue and grants

Tax and nontax revenue

Total expenditure

Net lending (+) / borrowing (-)

$\begin{array}{rrrrrr}-5.4 & 12.1 & -0.6 & 6.1 & -0.8 & 7.6\end{array}$

$\begin{array}{llllll}25.1 & 30.7 & 28.6 & 14.6 & 23.3 & 11.4\end{array}$

$\begin{array}{llllll}7.4 & 8.0 & 5.4 & 2.6 & 2.2 & 2.2\end{array}$

$\begin{array}{llllll}17.0 & 24.1 & 23.3 & 12.5 & 21.1 & 9.1\end{array}$

$\begin{array}{llllll}19.4 & 6.1 & 18.4 & 16.1 & 12.7 & 11.6\end{array}$

$\begin{array}{llllll}5.7 & 0.6 & 28.1 & 9.3 & 16.4 & 13.3\end{array}$

(Percent of GDP)

External sector

Current account balance

Overall balance of payments

$\begin{array}{rrrrrr}-9.4 & 12.3 & 11.6 & -11.0 & -10.8 & -11.1 \\ 0.7 & -1.6 & -0.3 & -0.3 & -2.2 & -0.9\end{array}$

$\begin{array}{rrrrrr}37.3 & 36.1 & 30.8 & 30.4 & 29.9 & 28.6 \\ 14.6 & 15.4 & 15.1 & 15.5 & 15.6 & 15.6 \\ 41.0 & 40.0 & 33.6 & 34.9 & 34.8 & 32.5 \\ -3.6 & -4.0 & -2.7 & -4.6 & -4.9 & -3.9\end{array}$


Burundi: Selected Economic and Financial Indicators, 2010-15

\begin{tabular}{|c|c|c|c|c|c|c|}
\hline & 2010 & 2011 & 2012 & 2013 & 2014 & 2015 \\
\hline & & Prel. & \multicolumn{4}{|c|}{ Proj. } \\
\hline & \multicolumn{6}{|c|}{ (Percent of GDP) } \\
\hline Savings-investment balance & -9.4 & 12.3 & 11.6 & -11.0 & -10.8 & -11.1 \\
\hline Private & -5.8 & -8.3 & -8.8 & -6.5 & -5.9 & -7.2 \\
\hline Public & -3.6 & -4.0 & -2.7 & -4.6 & -4.9 & -3.9 \\
\hline \multicolumn{7}{|l|}{ External sector } \\
\hline Gross official reserves (US\$ million) & 332 & 296 & 288 & 319 & 308 & 342 \\
\hline Months of imports & 4.4 & 4.0 & 3.8 & 4.2 & 3.9 & 4.7 \\
\hline Debt-service to exports ratio (percent) & 1.4 & 3.3 & 3.8 & 7.2 & 10.6 & 13.1 \\
\hline \multicolumn{7}{|l|}{ Memorandum item: } \\
\hline Nominal Exchange rate (BIF/USD) & 1231 & 1261 & $\ldots$ & $\ldots$ & $\ldots$ & $\ldots$ \\
\hline GDP at current market prices (BIF billion) & 2495 & 2971 & 3566 & 4138 & 4663 & 5203 \\
\hline Nominal GDP per Capita (US Dollars) & 242 & 255 & 289 & 311 & 332 & 349 \\
\hline
\end{tabular}

Sources: Burundi authorities; IMF staff estimates and projections. 
July 27, 2012

\section{IMF Executive Board Completes First Review Under the Extended Credit Facility Arrangement for Burundi and Approves US\$6 Million Disbursement}

The Executive Board of the International Monetary Fund (IMF) today completed the first review of Burundi's performance under the program supported by the Extended Credit Facility (ECF). In completing the review, the Executive Board approved a modification of performance criteria for end-September 2012.

The Executive Board's decision will allow for the disbursement of an amount equivalent to SDR 4 million (about US\$ 6 million), bringing disbursements under the arrangement to an amount equivalent to SDR 5 million (about US\$ 7.6 million). Burundi’s current three-year ECF arrangement was approved on January 27, 2012 (See Press Release No.12/35)

Following the Executive Board's decision on Burundi, Mr. Naoyuki Shinohara, Deputy Managing Director and Acting Chair, issued the following statement:

"Progress under the Fund-supported program has continued at an uneven pace. Economic growth is expected to exceed 4 percent in 2012, but inflation has been persistent in the wake of the food and fuel price shocks. The medium-term economic outlook remains challenging and vulnerable to 
downside risks stemming from internal strains, declining donor support, and the external environment.

"Fiscal slippages in early 2012 were addressed decisively in a supplementary budget through a combination of revenue and expenditure measures. To ensure that the budget targets are achieved, further revenue mobilization, including through improvements in tax administration, remains important.

"Underlying inflation has started to decline, but monetary policy should stay focused on anchoring inflation expectations. Higher policy rates have contributed to a welcome slowdown in credit expansion, but further action will be necessary if inflationary pressures do not ease in the period ahead.

"Maintaining debt sustainability remains a priority for fiscal policy. Given Burundi's vulnerability to debt distress, financing should continue to rely primarily on highly concessional resources. Equally important, further steps are needed to strengthen public debt management, including the finalization of a medium-term debt management strategy currently under preparation.

"Burundi's competitiveness needs to be enhanced to boost growth prospects and accelerate economic development. To this end, it is important to push ahead with structural reforms to improve the business environment, including by strengthening infrastructure and energy supply," Mr. Shinohara added. 


\section{Statement by Mr. Momodou Saho, Alternate Executive Director for The Republic of Burundi}

July 27, 2012

1. My Burundian authorities are committed to strengthening the country's macroeconomic fundamentals and to continue the implementation of sound policies and reforms under their economic program. They are confident that this will contribute to consolidate economic and political gains, promote new growth drivers and inclusive growth, reduce poverty and help shield the economy from exogenous shocks. They are, however, mindful that internal and external constraints could hamper the success of their economic program. Nevertheless, with their strong policy commitment and continued support from the Fund and their development partners, my authorities are confident that they will succeed in implementing their economic program.

2. My authorities are appreciative of the Fund's constructive engagement and support under the ECF, and thank staff for the helpful policy dialogue and advice. Going forward, they are determined to further strengthen this engagement as they make progress toward achieving their key national and regional objectives as set out in the PRSP II and the EAC protocols.

3. My authorities have made steady progress in the implementation of the program despite the difficult global environment. All performance criteria and indicative targets for end-March 2012 were met, and the fiscal slippages have been adequately addressed through the revised 2012 budget. In view of this strong performance, my authorities request Directors' support for the conclusion of the first review under the ECF, and modification of performance criteria for end-September 2012.

\section{Recent economic and policy developments}

4. Burundi's economic activity continues to be adversely affected by protracted high fuel and food prices, poor weather conditions and declining donor flows. As a result, real GDP growth forecast for 2012 has been revised downwards to 4.2 percent from the earlier forecast of 4.8 percent. The expansion of services and foreign investments in the secondary 
sector remained the major drivers of growth while agricultural output was weak on account of drought conditions. Consequently, prices of foodstuffs rose sharply in addition to the continued increase in fuel prices. The authorities have also adjusted electricity and water tariffs. As a result, inflation rose sharply to 25 percent by end-March 2012 .

5. The authorities' 2011 fiscal outturn reflected progress made in strengthening their fiscal framework in line with the program targets. In this regard, domestic revenue mobilization improved and expenditure was kept within the program envelope. Although expenditure fell short of projection, the authorities did maintain pro-poor spending at the targeted level. Subsequently, to further protect the vulnerable, the authorities extended tax relief by eliminating custom duties, VAT, and administrative charges on 13 imported and locally produced food products amounting to 0.3 percent of GDP. Additionally, the authorities waived excise taxes on oil products to offset soaring oil prices and its effect on inflation. In response to declining external budget support, and the reduction of the revenue collections on foodstuffs and oil products, the government reviewed the 2012 budget with further expenditure cuts and increasing domestic revenue by introducing new taxes. These budget adjustments also respond to the program's goals.

6. Monetary policy has been tightened further to curb inflation. Consequently, the rate of growth of credit to the economy has moderated. Supervision of financial institutions has been improved and the Central Bank (BRB) continues to implement measures aimed at strengthening its financial safeguards. In this vein, it has established an audit committee and adopted a new audit charter that conforms to best practice. The BRB also continues to pursue a flexible exchange rate policy in order to mitigate external shocks. However, given the recent sharp depreciation of the domestic currency, it intervened in the foreign exchange market to reduce volatility.

7. On the external side, the current account widened owing to the deterioration of the terms of trade, the decline in external budget support and the high prices of imported food and fuel. As a result, official reserves fell to 4 months of import cover from 4.4 months in December 2011. 
8. My authorities completed preparation of the second Poverty Reduction and Growth Strategy Paper (PRSP-II) which focuses on the transformation of the Burundian economy. The key pillars of the strategy include the consolidation of good governance, achieving sustainable growth and job-creation, strengthening of national solidarity and improving management of land and the environment. The growth projections in the PSRP-II are necessarily ambitious, because of the transformative nature of its objectives. However, the authorities, like staff, are aware of the risks to the outlook. These relate to the ongoing international economic crisis, low international coffee prices and shortfalls in budget support. The authorities will take measures as necessary and in consultation with the Fund, to adjust policies in the event the adverse scenarios materialize. The authorities intend to organize an international conference of donors in October 2012 on PRSP-II.

\section{Policy framework and reforms going forward}

\section{Fiscal policy and reforms}

9. The authorities have made substantial progress with regard to the implementation of public financial management reforms. Significant gains were achieved in the areas of budget credibility, supervision, cash management, and government accounting. Going forward, they remain committed to further strengthening public financial management and, in this regard, intend to develop and implement a new public financial management strategy for 2012-2014 in order to consolidate the gains attained thus far. The legal and institutional framework will be improved and strengthened for more effective financial governance, promoting transparency and accountability. They have also simplified customs clearance procedures and created a one-stop border post with Tanzania, the main entry point of Burundi's foreign trade.

10. Given the downward trend in external aid inflows, the authorities are determined to further strengthen their capacity to mobilize domestic resources by enhancing tax administration, and widening the tax base. Critical steps will be taken to close fiscal loopholes in order to maximize tax collection. The authorities welcome IMF technical assistance in assessing the tax exemption system and fuel pricing mechanism, and look forward to further support going forward. 
11. My authorities are mindful of the country's risk of debt distress and, in this regard, they remain committed to pursuing the mobilization of concessional external financing. The authorities are also committed to completing and fully implementing their medium-term debt management strategy focused on improving the legal framework governing the issuance of guarantees and on lending; strengthening the debt management structure and building capacity for the analysis of cost indicators and basic debt risks. They intend to create a high-level committee to coordinate public debt management processes and to ensure that total public debt is consistent with the country's macroeconomic objectives.

\section{Monetary policy}

12. The authorities' monetary policy framework will continue to be focused on curbing inflation and streamlining credit to the private sector. The authorities believe that the flexible exchange rate has helped to stabilize the BRB's reserves and improve the liquidity of the financial sector. Nonetheless, they are concerned about the repercussions of this policy stance on inflation especially with the recent depreciation of the exchange rate.

\section{Financial sector reforms}

13. The BRB will continue strengthening its supervisory and regulatory services to the financial institutions with a view to broadening access to financial services under its inclusive finance framework. The BRB will also continue strengthening its internal control and risk management systems, and the modernization of its payment systems. Mobile banking will be a key pillar for deepening inclusive finance. Therefore, the legal and regulatory framework will be implemented. Furthermore, in the spirit of the strengthening investor protection and deepening of the financial sector, the central bank will strengthen the creditor rights framework.

\section{Other structural reforms}

14. Given the good progress in improving the business climate, "Doing Business 2012" rated Burundi the seventh most improved economy in the world. However, challenges in this

area remain. In that regard, the authorities will pursue efforts to improve the investment 99 
climate, strengthen the capacity of the Export and Investment Promotion agency and consolidate "one stop shop" for starting business, enhance customer services and implement the private and public partnership framework.

15. The authorities are pursuing implementation of deeper reforms in the coffee sector, energy and the privatization of public enterprises. The reforms in the mineral sector are ongoing guided by the modernization of the mineral code. With regard to its membership of the East African Community, reforms will be focused on enhancing of the economy's competitiveness.

\section{Conclusion}

16. The implementation of the ECF-supported program could be hampered by internal and external environment challenges. The authorities will maintain an appropriate mix of fiscal and monetary policies, as well as deepened structural reforms to support growth and reduce poverty. Given the large financial needs, the authorities count on the catalytic impact of IMF engagement to support Burundi in the mobilization of grants and high concessional resources. With their policy stance and continued support from the Fund and other development partners, my authorities are confident that they will succeed in achieving the objectives of their economic program. 